\title{
Transient regulation of focal adhesion via Tensin3 is required for nascent oligodendrocyte differentiation
}

\author{
Emeric Merour ${ }^{1 *}$, Hatem Hmidan ${ }^{1 *}$, Corentine Marie ${ }^{1}$, Pierre-Henri Helou ${ }^{1}$, Haiyang Lu ${ }^{1}$, \\ Antoine Potel ${ }^{1}$, Jean-Baptiste Hure ${ }^{1}$, Adrien Clavairoly ${ }^{1}$, Yi Ping Shih ${ }^{2}$, Salman Goudarzi ${ }^{3}$, \\ Sebastien Dussaud ${ }^{1}$, Philippe Ravassard ${ }^{1}$, Sassan Hafizi ${ }^{3}$, Su Hao Lo ${ }^{2}$, Bassem A. Hassan ${ }^{1}$, \\ and Carlos Parras ${ }^{1}$ \\ ${ }^{1}$ Paris Brain Institute, Sorbonne Université, Inserm U1127, CNRS UMR 7225, Hôpital Pitié-Salpêtrière, 75013 Paris, France. \\ ${ }^{2}$ Department of Biochemistry and Molecular Medicine, University of California-Davis, Sacramento, CA 95817, USA. \\ ${ }^{3}$ University of Portsmouth. School of Pharmacy and Biomedical Sciences, Portsmouth PO1 2DT, UK \\ * Equal contribution
}

Corresponding author: Carlos Parras carlos.parras@icm-institute.org

\section{ABSTRACT}

The differentiation of oligodendroglia from oligodendrocyte precursor cells (OPCs) to complex and extensive myelinating oligodendrocytes $(\mathrm{OLs})$ is a multistep process that involves largescale morphological changes with significant strain on the cytoskeleton. While key chromatin and transcriptional regulators of differentiation have been identified, their target genes responsible for the morphological changes occurring during OL myelination are still largely unknown. Here, we show that the regulator of focal adhesion, Tensin3 (Tns3), is a direct target gene of Olig2, Chd7, and Chd8, transcriptional regulators of OL differentiation. Tns3 is transiently upregulated and localized to cell processes of immature OLs, together with integrin- $\beta 1$, a key mediator of survival at this transient stage. Constitutive Tns3 loss-offunction leads to reduced viability in mouse and humans, with surviving knockout mice still expressing Tns3 in oligodendroglia. Acute deletion of Tns3 in vivo, either in postnatal neural stem cells (NSCS) or in OPCs, leads to a two-fold reduction in OL numbers. We find that the transient upregulation of Tns3 is required to protect differentiating OPCs and immature OLs from cell death by preventing the upregulation of $\mathrm{p} 53$, a key regulator of apoptosis. Altogether, our findings reveal a specific time window during which transcriptional upregulation of Tns3 in immature OLs is required for OL differentiation likely by mediating integrin- $\beta 1$ survival signaling to the actin cytoskeleton as OL undergo the large morphological changes required for their terminal differentiation. 


\section{INTRODUCTION}

Oligodendrocyte lineage cells, mainly constituted by oligodendrocyte precursor cells (OPCS) and oligodendrocytes (OLs), play key roles during brain development and neuronal support, by allowing saltatory conduction of myelinated axons and metabolically supporting these axons with lactate or glucose shuttling through the myelin sheath (Funfschilling et al., 2012; Lee et al., 2012; Meyer et al., 2018). Accumulating evidence also indicates their fundamental contribution to different aspects of adaptive myelination, a type of brain plasticity (Mount and Monje, 2017; Yang et al., 2020), shown by the requirement of new oligodendrogenesis for proper learning and memory in motor, spatial, and fear-conditioning learning paradigms (McKenzie et al., 2014; Xiao et al., 2016; Steadman et al., 2019; Pan et al., 2020; Wang et al., 2020; Xin and Chan, 2020). Furthermore, oligodendroglia and myelin pathologies have been recently linked, not only to the development of glioma (Liu et al., 2011), but to developmental (Castelijns et al., 2020; Phan et al., 2020), neurodegenerative (Grubman et al., 2019; Bryois et al., 2020), and psychiatric (Nott et al., 2019) diseases.

Unlike most precursor cells, OPCs constitute a stable population of the postnatal and adult CNS (Ffrench-Constant and Raff, 1986; Suzuki and Goldman, 2003). Therefore, OPCs need to keep a tight balance between proliferation, survival, and differentiation. This balance is crucial to maintain the OPC pool while contributing to myelin plasticity in adult life, and to remyelination in diseases such as multiple sclerosis (MS). Demyelinated MS plaques can be normally repaired in early stages of the disease, presumably by endogenous OPCs, but this repair process becomes increasingly inefficient with aging, when OPC differentiation seems to be partially impaired (Chang et al., 2002; Compston and Coles, 2002; Neumann et al., 2019). Therefore, understanding the mechanisms involved in OPC differentiation is critical to foster successful (re)myelination in myelin pathologies.

A large diversity of extrinsic signals, including those mediated by integrin signaling (reviewed in (Bergles and Richardson, 2016)) as well as many intrinsic factors, including transcription factors (TFs) and chromatin remodelers (reviewed in (Emery and Lu, 2015; Parras et al., 2020)), are involved in OPC proliferation, survival, and differentiation. However, the mechanisms for how these signals balance OPC behavior is largely unknown. OPC differentiation requires profound changes in chromatin and gene expression (Emery and Lu, 
2015; Küspert and Wegner, 2016; Wheeler and Fuss, 2016). TFs, such as Olig2, Sox10, Nkx2.2 or Ascl1, are key regulators of OL differentiation by directly controlling the transcription of genes implicated in this process (Qi et al., 2001; Stolt et al., 2002; Nakatani et al., 2013; Yu et al., 2013) but being already expressed at the OPC stage, it is still unclear how these TFs control the induction of differentiation. A growing body of evidence suggests that some of these TFs work together with chromatin remodeling factors during transcriptional initiation/elongation to drive robust transcription (Zaret and Mango, 2016). Accordingly, Olig2 and Sox10 TFs have been shown to cooperate with chromatin remodelers such as Brg1 (Yu et al., 2013), Chd7 (He et al., 2016; Marie et al., 2018), Chd8 (Marie et al., 2018; Zhao et al., 2018), and EP400 (Elsesser et al., 2019), to promote the expression of OL differentiation genes. To improve our understanding of the mechanisms of OL differentiation, we searched for novel common targets of these key regulators, by generating and analyzing the common binding profiles of Olig2, Chd7, and Chd8, in gene regulatory elements of differentiating oligodendroglia. We identified Tns3, coding for the focal adhesion protein Tensin3, as one such target and showed that it is expressed in immature OLs during myelination and remyelination, thus constituting a marker for this transient oligodendroglial stage. Using different genetic strategies to induce Tns3 loss-of-function mutations in vivo, we describe, the function of a Tensin family member in the CNS, demonstrating that Tns3 is required for oligodendrocyte differentiation in the postnatal mouse brain, at least in part by mediating integrin- $\beta 1$ signaling, essential for survival of differentiating oligodendroglia (Colognato et al., 2002; Benninger et al., 2006).

\section{RESULTS}

\section{Tns3 is a direct target gene of key regulators of oligodendrocyte differentiation}

To find new factors involved in oligodendrocyte differentiation, we screened for target genes of Olig2, Chd7, and Chd8, key regulators of oligodendrogenesis (Lu et al., 2000; Lu et al., 2002; Yu et al., 2013; He et al., 2016; Marie et al., 2018; Zhao et al., 2018; Parras et al., 2020). We generated and compared the genome-wide binding profiles for these factors in acutely purified oligodendroglial cells from postnatal mouse brain cortices by magnetic cell sorting (MACS) of $4^{+}$cells (Marie et al., 2018). MACS-purified cells, composed of $80 \%$ PDGFR $\alpha^{+}$OPCs and $20 \%$ of $\mathrm{Nkx2.2} / \mathrm{CC1}^{+}$immature oligodendrocytes (iOLs), were subjected to chromatin immunoprecipitation followed by sequencing (ChIP-seq) for Olig2 and histone modifications marking the transcription activity of gene regulatory elements (H3K4me3, H3K4me1, 
H3K27me3, and H3K27ac; Fig. 1A). The profile of activity histone marks at Olig2 binding sites indicated that Olig2 binds promoters (60\%) and enhancers (40\%) with either active or more poised/repressive states (Fig. S1A-F), supporting the suggested pioneer function of Olig2 in oligodendrogenesis ( $\mathrm{Yu}$ et al., 2013). Among the 16,578 chromatin sites bound by Olig2 corresponding to 8,672 genes (Fig. S1D), there were key regulators of oligodendrocyte differentiation, including Ascl1, Sox10, Myrf, Chd8, and Smarca4/Brg1 (Fig. 1B; supplementary table 1). Combining Olig2 with Chd7 and Chd8 binding profiles, that we previously generated using the same protocol (Marie et al., 2018), we found 1774 chromatin sites commonly bound by the three regulators, with half of them ( $47 \%$ and 832 peaks) corresponding to active promoters (H3K4me3/H3K27ac marks) of 654 protein-coding genes (Fig. 1C, supplementary table 2). Among these genes, Tns3 coding for Tensin3, a focal adhesion protein deregulated in certain cancers (Martuszewska et al., 2009), showed the highest expression levels in iOLs relative to other brain cell types (Zhang et al., 2014; Fig. 1D). Indeed, Olig2, Chd7, and Chd8 commonly bound three putative promoters of Tns3 having active transcription marks in purified oligodendroglia (H3K27ac/H3K24me3; Fig. 1E). To directly assess whether Tns3 expression requires the activity of these key regulators, we interrogated the transcriptomes of these oligodendroglial cells purified from Chd7iKO (Pdgfra-CreER $R^{T}$ Chdfflox/flox), Chd8cKO (Olig1 ${ }^{\text {Cre }}$; Chd $8^{\text {flox/flox }}$ ), and their respective control cortices (Marie et al., 2018; Zhao et al., 2018). Tns3 transcripts were largely downregulated upon acute deletion of these factors in postnatal OPCs/iOLs (Fig. 1F,G), indicating that Tns3 expression in OPCs/iOLs is directly controlled by Chd7 and Chd8 chromatin remodelers, key regulators of oligodendrocyte differentiation.

\section{Tns3 transcripts are highly expressed in mouse and human immature oligodendrocytes}

We then investigated Tns3 expression pattern in the brain. High expression levels of Tns3 transcripts in iOLs, compared to its low expression in other cells of the postnatal mouse brain detected by bulk transcriptomics (Fig S2A; Zhang et al., 2014) were paralleled by the sparse labelling with Tns3 probes enriched in the white matter of the postnatal and adult brain detected by in situ hybridization (Fig. S2B; Allen Brain atlas, https://portal.brain-map.org/. By harnessing single-cell transcriptomics (scRNA-seq), we sought to create an integrative gene profiling for oligodendrocyte lineage cells, by bioinformatics integration and analyses of oligodendrocyte lineage cells at embryonic, postnatal, and adult stages (Marques et al., 2016, 
2018). We thus integrated these datasets using Seurat (Stuart et al., 2019) and selected 5,516 progenitor and oligodendroglial cells. Unsupervised clustering and visualization of cells in two dimensions with uniform manifold approximation and projection (UMAP), identified nine different clusters following a differentiation trajectory. Based on known cell-subtype specific markers (Fig. S2E and supplementary Table3), we could identify these clusters as (Fig. S2C,E): (1) two types of neural stem/progenitor cells, that we named NSCs and NPCs according to their expression of stem cell (Vim, Hes1, Id1) and neural progenitor (Sox11, Sox4, Dcx) markers; (2) OPCs expressing their known markers (Pdgfra, Cspg4, Ascl1) and cycling OPCs also enriched in cell cycle markers (Mki67, Pcna, Top2); (3) two stages of iOLs, both expressing the recently proposed markers Itpr2 and Enpp6 (Marques et al., 2016; Xiao et al., 2016), and which are split by the expression of $N k \times 2-2$ (iOL1 being $N k \times 2-2^{+}$and iOL2 being $N k \times 2-2^{-}$), in agreement with our previous characterization by immunofluorescence (Nakatani et al., 2013; Marie et al., 2018); (4) myelin forming oligodendrocytes (MFOLs), enriched in markers such as Slc9a3r2 and Igsf8; and (5) two clusters of myelinating OLs, that we named MOL1 and MOL2, expressing transcripts of myelin proteins (Cnp, Mag, Mbp, Plp1, Mog) and some specific markers of each cluster, including Mgst3, Pmp22 for MOL1 (corresponding to MOL1/2/3/4 clusters of Marques 2016), and Neat1, Grm3, I/33 for MOL2 (corresponding to MOL5/6 clusters of Marques 2016). Interestingly, Tns3 transcripts were strongly expressed in both iOL1 and iOL2 clusters (Fig. S2D,E), similar to the recently proposed iOL markers Itpr2 and Enpp6 (Fig. $\mathrm{S} 2 \mathrm{E})$, and downregulated in mature/terminally differentiated oligodendrocytes, indicating that high levels of Tns3 expression are specific to iOLs. We finally, assessed whether Tns3 expression pattern was conserved in human oligodendroglia pursuing a similar bioinformatics analysis using single cell transcriptomes from human oligodendroglia differentiated from embryonic stem cells (Chamling et al., 2021). Upon integration with Seurat and identification of cluster cell types using specific markers, we selected 7,690 progenitor and oligodendroglial cells that corresponded to six main cluster cell types following a differentiation trajectory from neural cells (NSCs) up to immature OLs (iOL1 and iOL2), as depicted by UMAP representation (Fig. S2F,H). Cells expressing high levels of TNS3 corresponded to immature oligodendrocytes (iOL1 and iOL2 clusters, Fig. S2G,H), indicating a conserved expression pattern of Tns3/TNS3 between mouse and human oligodendrogenesis. 
bioRxiv preprint doi: https://doi.org/10.1101/2022.02.25.481980; this version posted February 27, 2022. The copyright holder for this preprint (which was not certified by peer review) is the author/funder, who has granted bioRxiv a license to display the preprint in perpetuity. It is made available under aCC-BY-NC-ND 4.0 International license.

\section{Tns3 protein is enriched in the cytoplasm and processes of immature oligodendrocytes}

Given the high expression level of Tns3 transcripts in immature oligodendrocytes, we characterized the Tns3 protein expression pattern in the postnatal brain using commercial and homemade Tns3-recognizing antibodies. Optimization of immunofluorescence protocols demonstrated signal in $\mathrm{CC}^{+}$oligodendrocytes in the postnatal brain with four different antibodies (P24, Fig. S3). To our surprise, while all antibodies showed signal localized in the cytoplasm and main processes of CC1+ OLs (Fig. S3A-E), one Tns3-recognizing antibody (Millipore) also presented a strong nuclear signal never reported for Tns3 localization in other tissues (such as lung, liver, and intestine) (Katz et al., 2007; Nishino et al., 2012; Cao et al., 2015). To better characterize Tns3 protein expression pattern and its subcellular localization, we generated a knock-in mice tagging the Tns3 C-terminal side with a V5-tag (Tns $3^{\text {Tns3-V5 }}$ mice) by microinjecting mouse zygotes with a single strand oligodeoxynucleotide (ssODN) containing V5 sequence together with Cas9 protein and a gRNA targeting the stop codon region of Tns3 (Methods; Fig. S4A-C). We first verified by immunofluorescence that Tns3-V5 protein in $T n s 3^{\text {Tns3-V5 }}$ mice presented the expression pattern reported for Tns3 in the lung and the kidney (Fig. S4D,E). We then characterized Tns3 protein expression in oligodendroglia using V5 antibodies, finding that Tns3 protein can be detected at high levels in the cytoplasm and main processes of $\mathrm{CC}^{+}$iOLs but not in their nuclei (Fig. 2A). Using an antibody recognizing Itpr2, a suggested iOL marker (Marques et al., 2016), we saw that Tns3 largely overlapped with Itpr2 (Fig. 2B). Using Nkx2.2 and Olig1 1ytoplamic expression distinguishing iOL1 and iOL2

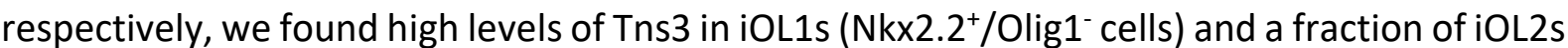
(Nkx2.2\% /Olig1 $1^{\text {cytoplamic }}$ cells; Fig. $2 \mathrm{C}$ ), suggesting that Tns3 protein expression peaks in early iOLs. Comparison with Opalin protein localized in the cell body, processes, and myelin segments of oligodendrocytes, showed that Tns3 levels decreased with increasing levels of Opalin, with Tns3-V5 levels undetectable in myelinating oligodendrocytes (i.e. Opalin ${ }^{+} / \mathrm{CC}^{+}$ cells presenting myelinated segments; Fig. 2D, arrowheads). We then performed Western blot analysis with anti-V5 antibodies in purified $\mathrm{O}^{+}$cells from P7, P14, and P21 Tns $3^{\text {Tns3-V5 }}{ }^{\text {mouse }}$ brains to assess their specificity to recognize Tns3-V5, knowing that two Tns3 isoforms can be detected at the transcript level in the human brain (Fig. S4F, GTEX project, gtexportal.org/home/gene/TNS3). Indeed, we could detect both the full-length (1450 aa, 155 $\mathrm{kDa}$ ) and the Tns3 short (C-term, $61 \mathrm{kDa}$ ) isoforms in 04+ cells from brains at P7 and P14 stages having many iOLs, but not at P21 having mainly mOLs (Fig. S4G,H), thus validating the 
specificity of the anti-V5 antibodies in recognizing Tns3 protein. We eventually found a Tns3 antibody also recognizing the C-terminal of Tns3 protein (Sigma $\mathrm{Ct}$ ) that upon optimized immunofluorescence labeling confirmed the Tns3 expression pattern seen with the V5 antibodies. In combination with Nkx2.2 and Olig1 immunofluorescence, it showed that Tns3 is strongly detected in the cytoplasm and main cellular processes of all iOL1s, defined as

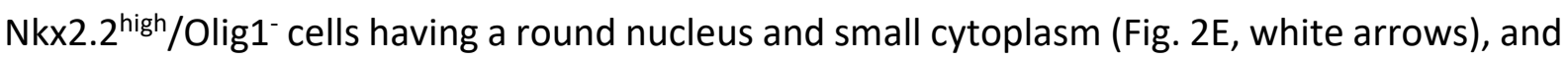
it divided iOL2s, defined as Nkx2.2\% $/ \mathrm{CC} 1^{\text {high }}$ cells, into three stages: (1) Tns3 $3^{\text {high }} / \mathrm{Nkx2.2} / \mathrm{Olig} 1^{-}$

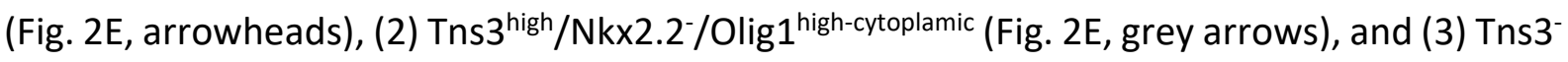
/Nkx2.2\% Olig1 ${ }^{\text {high-cytoplamic }}$ (Fig. 2E). A similar Tns3 expression pattern and localization was found in vitro using neonatal neural progenitors' differentiating cultures, where Tns3 was detected together with CNP myelin protein in the cytoplasm and cell processes of $\mathrm{Nk} \times 2.2^{\text {high }} / \mathrm{CNP}^{+}$differentiating oligodendrocytes (Fig. 2F,G). Altogether, these results indicate that high but transient levels of Tns3 protein characterize early immature oligodendrocytes (iOL1s and early iOL2s), being localized at their cytoplasm and cell processes (Fig. $2 \mathrm{H}, \mathrm{I}$ ).

Finally, we investigated whether other Tensin family members were expressed in oligodendroglia, finding that Tns1 and Tns2 but not Tns4 were detectable at low levels in iOLs by immunofluorescence (Fig. S5A,B), paralleling their low transcription levels compared to Tns3 (Fig. S5C; brainrnaseq.org). Therefore, Tns3 appears to be the main Tensin expressed during oligodendrocyte differentiation, suggesting that Tns3 function in immature oligodendrocytes is likely to be evolutionarily selected, and thus of biological importance in oligodendrogenesis.

\section{Tns3 expression is found in immature oligodendrocytes during remyelination}

Given the strong Tns3 expression in iOLs during postnatal myelination, we hypothesized that Tns3 expression could be enriched during remyelination in newly formed oligodendrocytes contributing to remyelination. To test this hypothesis, we performed lysolecithin (LPC) focal demyelinating lesions in the corpus callosum of adult (P90) Tns $3^{\text {Tns3-V5 }}$ and wild-type mice, and assessed for Tns3 expression at the peak of oligodendrocyte differentiation ( 8 days postlesion) in this remyelinating model (Nait-Oumesmar et al., 1999). We found that while nonlesioned adult brain regions contained only sparse $\mathrm{Tns}^{+}$iOLs (CC1 ${ }^{\text {high }} / \mathrm{Olig} 1^{\text {cyto-high }}$ cells), remarkably many $\mathrm{Tns}^{+}$iOLs were detected in the remyelinating area using both V5 (Fig. S6A,C, arrows) and Tns3 antibodies (Fig. S6B, arrows). Quantification of Tns3 ${ }^{+}$cells showed a 
clear increase in $\mathrm{Tns}^{+}$iOLs around the lesion borders compared to the corpus callosum far from the lesion area (Fig. S6D), suggesting that Tns3 expression may be a useful marker of ongoing remyelination and lesion repair. Of note, we could also detect Tns3 expression in some microglia/macrophages in the lesion area using a combination of F4/80 antibodies (Fig. S6C, arrowheads). Altogether, all these data indicate that Tns3 expression peaks at the onset of oligodendrocyte differentiation, labeling immature oligodendrocytes during both myelination and remyelination.

\section{Tns3 knockout mice present normal numbers of oligodendroglia in the postnatal brain and still express Tns 3 full length transcripts}

To explore the role of Tns3 in OL differentiation, we first analyzed a Tns3 gene trap mouse line $\left(\right.$ Tns $3^{\beta g e o}$ ) previously studied outside the CNS (Chiang et al., 2005), where the $\beta g e o$ cassette is inserted after Tns3 exon 4 (Fig. S7A) driving LacZ transcription and by inserting a stop poly-A sequence, predicted to be a Tns3 loss-of-function mutation. Despite the original report of postnatal growth retardation in Tns $3^{\beta g e o / \beta g e o}$ mice, these mice were kept in homozygosity for several generations in C57BL/6 genetic background (Su-Hao Lo, UC Davis). We thus analyzed the impact in oligodendrogenesis in the postnatal brain of Tns $3^{\beta g e o}$ animals. We first immunodetected $\beta$ galactosidase in OLs (Olig2 ${ }^{+} / \mathrm{CC}^{+}$and Olig2 ${ }^{+} / \mathrm{PDGFR}^{-}$cells) of $\mathrm{Tns} 3^{\beta g e o}$ postnatal brains at P21 (Fig. S7B,C), paralleling our characterization of Tns3 expression with V5 and Tns3 antibodies. We then quantified the density of PDGFR $\alpha^{+}$OPCs or $\mathrm{CC} 1^{+}$ oligodendrocytes in Tns $3^{\beta g e o / \beta g e o}$ and $T n s 3^{\beta g e o /+}$ littermates at P21, finding similar number of OPCs and oligodendrocytes in two main white matter areas (corpus callosum and fimbria; Fig. 3A-B). Moreover, quantification of three different stages of oligodendrocyte differentiation (iOL1, iOL2, and $\mathrm{mOL}$ ) by Olig2/CC1/Olig1 immunofluorescence did not reveal changes in the rate of oligodendrocyte differentiation (proportion of each stage) in Tns $3^{\beta g e o / \beta g e o}$ mice compared to control littermates (Fig. 3C-D). We verified the homozygosity of Tns $3^{\beta g e o}$ allele in Tns3 $3^{\beta g e o / \beta g e o}$ mice by PCR amplification from genomic DNA of P21 brains finding that primers recognizing Intron 4 and $\beta$ geo only produced PCR amplicons in Tns $3^{\beta g e o / \beta g e o}$ mice but not when using Intron 4 flanking primers that only produced PCR amplicons in wild type mice; Fig. S7D). We then checked for Tns3 full-length transcripts using cDNA generated from P21 brains, and to our surprise, primers flanking Exons 17 and 31 were similarly amplified from cDNA of 
Tns $3^{\beta g e o / \beta g e o}$ and wild type brains (Fig. S7E), suggesting that in the brain of Tns $3^{\beta g e o / \beta g e o}$ mice Tns3 full-length transcripts coding for Tns3 protein are still produced. Altogether, these results suggested that, unlike in other tissues (Chiang et al., 2005), the Tns3 $3^{\beta g e o}$ allele does not lead to Tns3 loss-of-function in the brain, likely through the generation of alternative spliced Tns3 variants, and is thus not suitable for assessing Tns3 function in the CNS.

We therefore generated new Tns3 knockout mouse using CRISPR/Cas9 technology, by introducing loss-of-function mutations (indels) at the beginning of Tns3 full-length coding sequence. We generated CRISPR integrative plasmids (Fig. S8A) driving Cas9 expression and gRNAs targeting Tns3 Exon- 6 at the levels of the first coding ATG, using as control, plasmids without the Tns3-targeting sequence of the gRNA. Strong cutting efficiency of two gRNAs was validated by lipofection of neural progenitors (Fig. S8B-F). We then used these optimized tools to induce CRISPR-mediated Tns3 mutations in mouse zygotes (Methods), generating and characterizing two mouse lines having small deletions (4-deletion and 14-deletion) after the first coding ATG of Tns3 (Fig. 3E), expected to cause frame shifts leading to Tns3 loss-offunction. Remarkably, homozygous animals were found in reduced numbers compared to mendelian ratios with many of them dying during embryonic development (Fig. 3F) with most homozygous animals showing major growth retardation by the second postnatal week compared with their littermates (Fig. 3G), similar to the original report of Tns $3^{\beta g e o}$ mice (Chiang et al., 2005). Furthermore, we could still immunodetect Tns3 protein in $\mathrm{CC}^{+}$oligodendrocytes of these homozygous mice at P21 with at least two different Tns3 antibodies (Fig. 3H,I), and detect Tns3 exons corresponding to Tns3 full-length transcript by qPCR (Fig. 3J). Further analysis of these mice was prevented by the Covid-19 lockdown leading to the loss of these Tns3 knockout mouse lines. Altogether, these results suggest that mice carrying constitutive Tns3 loss-of-function mutations seems to escape the full Tns3 loss-of-function in the brain, by generating alternative spliced variants containing the main Tns3 full-length exons, and thus we considered these animals not suitable to study Tns3 function in oligodendrogenesis.

Finally, to assess whether TNS3 is potentially required during human development, we explore for the presence of TNS3 gene variants in the human population using the gnomAD database containing 125,748 exomes and 15,708 whole-genome sequences from unrelated individuals (Karczewski et al., 2020; Lek et al., 2016). Homozygous predicted loss-of-function (pLoF) alleles of TNS3 were not found, and heterozygous pLoF were greatly below the 
expected frequency ( 0.1 observed/expected ratio, with $90 \%$ confidence interval of $0.05-0.19$; and LOEUF of 0.19; Fig. S9A; https://gnomad.broadinstitute.org), meaning that heterozygous loss-of-function variants of TNS3 causes $\sim 80 \%$ developmental mortality, a rate similarly high to key neurodevelopmental genes such as SOX10 (LOEUF=0.21; Fig. S9B), CHD7 (LOEUF=0.08; Fig. S9C), and CHD8 (LOEUF=0.08; Fig. S9D), contrary to less broadly required factors such as NKX2-2 (LOEUF=0.67; Fig. S9E) and OLIG1 (LOEUF=1.08; Fig. S9F). Therefore, TNS3 loss-offunction variants are badly tolerated in both mouse and human development.

\section{In vivo CRISPR-mediated Tns3 loss-of-function in neonatal neural stem cells impairs}

\section{oligodendrocyte differentiation}

Given the tendency of cells to escape the Tns3 loss-of-function upon constitutive knockout mutations, we decided to assess Tns3 requirement during postnatal oligodendrogenesis by inducing in vivo acute Tns3-deletion in few neural stem cells (NSCs) of the neonatal brain and tracing their cell progeny with a GFP reporter. For this, we combined the postnatal electroporation technique with CRISPR/Cas9 technology. First, we used our previously validated gRNAs targeting Tns3 at the first coding ATG (exon 6; Fig. S8) inserting them in an integrative CRISPR/Cas9 plasmid also expressing the GFP reporter (Fig. 4A), to transfect neonatal NSCS of the dorsal subventricular zone (SVZ), which generate a large number of oligodendroglial cells during the first postnatal weeks (Kessaris et al., 2006; Nakatani et al., 2013), and focused our study on glial cells by quantifying the GFP ${ }^{+}$progeny of targeted NSCs, outside the SVZ and located in the dorsal telencephalon three weeks later (P22, Fig. 4B). The fate of $\mathrm{GFP}^{+}$cells was determined by immunodetection of GFP and glial subtype markers (CC1 $^{\text {high }}$ for oligodendrocytes, PDGFR $\alpha$ for OPCs, and CC1 ${ }^{\text {low }}$ and their unique branched morphology for astrocytes). Remarkably, brains electroporated with the CRISPR plasmids targeting Tns3 had a 2-fold reduction in $\mathrm{GFP}^{+}$oligodendrocytes compared to brains electroporated with control plasmids, while GFP+ OPCs were found in similar proportions (Fig. $\left.4 C, C^{\prime}, D\right)$. The proportion of $\mathrm{GFP}^{+}$astrocytes was increased by 1.5 -fold, likely as a result of the large reduction in oligodendrocytes, as the number of GFP+ astrocytes was not changed $(61.3$ \pm 10.9 in experimental versus $57.2 \pm 11.8$ in controls; Fig. $\left.4 C, C^{\prime}, D\right)$. To assess whether the reduction in oligodendrocytes from Tns3-deleted NSCs was the consequence of a reduction in OPCs generated, we assessed for possible changes in numbers, proliferation, and survival of 
OPC at P11, when most cortical OPCs have not yet started differentiation. We found no differences in the proportion of GFP cells being OPCs (Fig. 4E), nor the proliferative status of $\mathrm{GFP}^{+}$OPCs ( $\mathrm{MCM}^{+} / \mathrm{PDGFR}^{+}$cells; Fig. 4F) between experimental and control brains, while the reduction of oligodendrocytes was already marked (Fig. 4E), indicating that loss of Tns3 only affected the process of OPC differentiation into oligodendrocytes.

Given the expression of two Tns3 isoforms in the brain (Fig. S4F,G), we asked whether a deletion of both isoforms would have a greater impact in oligodendrocyte differentiation. We thus used two gRNAs efficiently cutting the beginning and the end of Tns 3 coding sequence (5'-3'gRNAs, Methods), to delete the whole Tns3 locus. We found a similar reduction of oligodendrocytes in the loss of the two Tns3 isoforms than in mutations affecting only fulllength Tns3 (Fig. $4 \mathrm{H}, \mathrm{H}^{\prime}, \mathrm{I}$ ), suggesting that the small Tns3 isoform does not play an additional role with full-length Tns3 in oligodendrocyte formation. Altogether, these results indicate that Tns3 loss-of-function mutations in neonatal SVZ-NSCs impair OPC differentiation without apparent changes in OPC generation and proliferation, thus suggesting that Tns3 is largely required for OPC differentiation into oligodendrocytes in the postnatal brain (Fig. 4G).

\section{OPC-specific Tns3 deletion impairs oligodendrocyte differentiation in the postnatal brain}

Given the heterogeneity of CRISPR/Cas9-mediated indels and the difficulties to assess in vivo the penetrance of their Tns3 loss-of-function, to address in more detail the consequences of Tns3 loss-of-function, we designed a Tns3 conditional knockout allele, by flanking with LoxP sites the exon 9 (Fig. S10A). In this Tns3-floxed allele (Tns $3^{\text {flox }}$ ), Cre-mediated recombination induces a transcription frame shift translated into an early stop codon, leading to a putative small peptide of 109 aa instead of the full length Tns3 protein (1442 aa; Fig. S10B,C). Mouse embryonic stem cells (ESCs) were transfected with a plasmid expressing Cas9, GFP, and gRNAs flanking Tns3 exon 9, and a Tns3-floxed targeting vector (Fig. S10D), in order to induce CRISPR/Cas9-mediated homologous recombination. After verifying the presence of Tns3floxed allele in Tns3 locus by Sanger sequencing, positive ESC clones were injected into blastocysts to generate Tns3-floxed (Tns $\left.3^{f l o x}\right)$ mice. Finally, we combined Tns $3^{f l o x}$ animals with Pdgfra-CreER ${ }^{T}$ mice, to induce Cre-mediated Tns3 deletion specifically in OPCs, in presence of a Cre-inducible reporter background (Rosa26 $6^{\text {stop-YFP }}$ ) in order to trace Tns3-deleted cells.

In order to specifically delete Tns3 in postnatal OPCs, we administered tamoxifen at P7 to Pdgfra-CreER ${ }^{T}$; Tns3flox/flox; Rosa26 $6^{\text {stop-YFP }}$ (hereafter called Tns3-iKO mice) and control pups 
(Pdgfra-CreER ${ }^{T}$; Tns3 ${ }^{+/+} ;$Rosa26 $6^{\text {stop-YFP }}$ littermates) and analyzed its effects on oligodendrogenesis at P14 and P21 (Fig. 5A) both in white matter (corpus callosum and fimbria) and grey matter regions (cortex and striatum). We first assessed for the efficiency of Tns3 deletion in $\mathrm{Nkx}^{2} .2^{+} / \mathrm{GFP}^{+}$iOLs from different regions by immunofluorescence using a Tns3 antibody (Sigma Ct), finding that the strong Tns3 signal present in Nkx2.2 $2^{+} \mathrm{GFP}^{+}$iOLs of control brains was almost completely eliminated in Tns3-iKO iOLs without affecting Tns3 expression in vessels (Fig. S11B, B',C, arrows and arrowheads versus asterisks). We then assessed for changes in oligodendrogenesis. Remarkably, the number of oligodendrocytes $\left(\mathrm{CC}^{+} / \mathrm{GFP}^{+}\right.$cells) was reduced by half in all quantified regions (reduction of $38.95 \%$ in the CC, $48.60 \%$ in cortex, $50.88 \%$ in the fimbria, $38 \%$ in the striatum; Fig. 5B-D, Fig. S11E-F', Fig. S12B,B') in Tns3-iKO compared to control, while OPC (PDGFR $\alpha^{+} / \mathrm{GFP}^{+}$cells) density was unchanged (Fig. 5B-D). Using markers distinguishing different stages of oligodendrocyte differentiation (iOL1 and $\mathrm{iOL2} / \mathrm{mOL}$ ), we found that the density of iOL1s (Nkx2.2 $2^{\text {high }}$ cells), which express the highest levels of Tns3 protein in control brains, was unchanged (Fig. $\left.\mathrm{S} 11 \mathrm{~B}, \mathrm{~B}^{\prime}, \mathrm{D}\right)$, while the density of early $\mathrm{iOL} 2 \mathrm{~s}\left(\mathrm{CC}^{+} / \mathrm{Olig} 1^{-}\right.$cells) and later oligodendrocyte stages (iOL2/mOLs CC1 $1^{+} / \mathrm{Olig}^{+}$cells), was reduced by $30 \%$ and $50 \%$ respectively in Tns3-iKO compared to controls (Fig. 5E-F), suggesting that Tns3 is required for normal oligodendrocyte differentiation. Finally, we assessed possible changes in OPC proliferation by immunodetection of $\mathrm{Mcm} 2$, finding no significant changes in the proliferation of Tns3-iKO OPCs compared to control OPCs (Fig. S12C,C',D).

Altogether, these results indicate that acute deletion of Tns3 in OPCs reduces by 2 -fold generation of oligodendrocytes in the postnatal brain, without major changes in OPC numbers and proliferation (Fig. 5G).

\section{Tns3-iKO oligodendroglia undergo apoptosis}

Tensins are known to mediate integrin stabilization and activation in other cells-types (Liao and Lo, 2021), with Tns3 been shown to bind integrin- $\beta 1$ through its phosphotyrosine-binding domain and FAK through its SH2 domain in fibroblasts (Cui et al., 2004; Liao et al., 2007; Georgiadou et al., 2017). In oligodendroglia, integrin $\alpha 6 \beta 1$ association with Fyn kinase is required to amplify PDGF survival signaling and to promote myelin membrane formation, by switching neuregulin signaling from a PI3K to a MAPK pathway (Colognato et al., 2004). Moreover, by conditional ablation of integrin- $\beta 1$ in vivo, it was demonstrated that integrin- $\beta 1$ 
signaling is involved in survival of differentiating oligodendroglia, but not required for axon ensheathment and myelination per se (Benninger et al., 2006). We therefore, investigated the expression of genes involved in integrin signaling in the transcriptome of oligodendroglial cells. Indeed, Tns3 expression pattern in iOLs was closely matching that of Itgb1 (integrin-b1), Fyn, Bcar1/p130Cas, and Ptk2/Fak both in mouse and human oligodendroglia (Fig. S13A,B). Furthermore, using neural progenitor differentiation cultures, we observed co-expression of integrin- $\beta 1$ and Tns3 in CNP+ oligodendrocytes by immunofluorescence (Fig. S13C), suggesting that Tns3 could relay integrin- $\beta 1$-mediated survival signal in differentiating oligodendroglia. Therefore, we assessed signs of cell death in Tns3-iKO oligodendroglia by performing the TUNEL technique together with GFP and CC1 immunodetection. Interestingly we found a 5fold increase in TUNEL ${ }^{+}$cells in the dorsal telencephalon of Tns3-iKO brain, compared to control, without significant changes in non-oligodendroglial cells present in the SVZ (Fig. 6AC). To gain more insight into the cellular alterations and cell death of Tns3-deleted oligodendroglia, we investigated their cellular morphology and behavior by video microscopy during their differentiation in culture. To this end, we MACS-purified OPCs from Tns3-iKO and control (Tns3 flox/flox; Rosa26 $6^{\text {stop-YFP }}$ littermates) mice at P7, two days after administration of tamoxifen, plated them in proliferating medium for three days, and recorded their behavior during three days in the presence of differentiation medium (Fig. 6D). Using the expression of the YFP as a readout of Cre-mediated recombination, we compared the behavior of YFP' ${ }^{+}$cells (Tns3-iKO) with neighboring YFP- cells (internal control) in the same cultures. In parallel, we used MACSorted cells from control mice as external control. Quantification of the proportion of $\mathrm{YFP}^{+}$cells over time showed a $20 \%$ reduction of $\mathrm{YFP}^{+}$cells (from $80 \%$ to $60 \%$ ) during the 3 days in proliferation medium followed by a reduction to $50 \%$ by day 3 in differentiation medium (Fig. 6E), suggesting possible cell death of Tns3-mutant cells. Live imaging monitoring of cell behavior showed that once $\mathrm{YFP}^{+}$cells had developed multiple branched morphology, characteristic of differentiating oligodendrocytes, they showed a 4-fold increase in their probability to die compared to $\mathrm{YFP}^{-}$cells of the same culture (Fig. 6F-H, yellow and white arrows, respectively) or to cells from control cultures, with more pronounced cell death by the third day of culture (Fig. 6F,G). Together, these results indicate that Tns3-iKO oligodendroglia present increased cell death both in vivo and in primary culture, at the stage when Tns3 is upregulated and cells start to developed their branched morphology, suggesting that Tns3 likely mediates $\beta 1$-integrin signaling required for their survival. 


\section{Apoptosis of Tns3-iKO oligodendroglia is mediated by p53 upregulation}

To study the molecular mechanisms of Tns3 function in oligodendroglia, we first looked at P53 expression, the master transcriptional regulator of the cellular genotoxic stress response (Kastenhuber and Lowe, 2017; Aubrey et al., 2018). Interestingly, we found a 10-fold increase

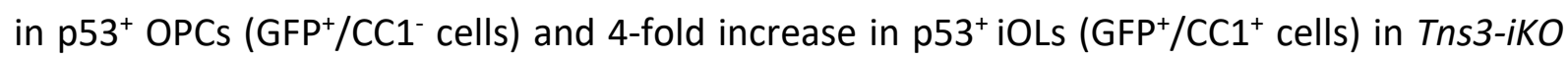
compared to control (Fig. 7A-C), suggesting that the loss of Tns3 leads to an upregulation of p53, that together with the loss of integrin- $\beta 1$ survival signal, mediates the cell death of Tns3iKO differentiating oligodendroglia (Fig. 7D).

\section{DISCUSSION}

The tight balance of oligodendrocyte precursor cells (OPCs) between proliferation, survival, and differentiation ensures their capacity to respond to the myelination needs of the CNS by generating new oligodendrocytes on demand, whilst avoiding the generation of brain gliomas through uncontrolled OPC proliferation. The observation that OPCs are present within demyelinating MS lesions, but fail to efficiently differentiate into myelinating cells with age and disease progression (Chang et al., 2002; Neumann et al., 2019), together with the strong sensitivity of immature oligodendrocytes to survival/apoptotic signals (Hughes and Stockton, 2021), suggests that efforts to foster OPC differentiation and survival of immature oligodendrocytes are a critical events for healthy aging and successful remyelination in MS patients. In this study, we combined the genome-wide binding profile of key regulators of oligodendrocyte differentiation, Olig2, Chd7, and Chd8 (Lu et al., 2000; Zhou et al., 2000; Lu et al., 2002; Zhou and Anderson, 2002; He et al., 2016; Küspert and Wegner, 2016; Marie et al., 2018; Zhao et al., 2018), to identify their common gene targets, and focused our analysis on Tensin3 (Tns3), whose expression matched the onset of oligodendrocyte differentiation. To study Tns3 expression and function, we generated several genetic tools, including CRISPR/Cas9 vectors to induce Tns3 mutations both in vivo and in vitro, a Tns $3^{\text {Tns3-V5 }}$ knock-in $^{\text {. }}$ mouse, two constitutive Tns3 knockout mice, and finally an inducible knockout (Tns3 $3^{\text {Flox }}$ ) mouse. Using these tools, we provide several lines of evidence showing that Tns3 is upregulated in immature oligodendrocytes (iOLs) and required for normal oligodendrocyte 
differentiation. First, we show that Tns3 expression is strongly induced at the onset of oligodendrocyte differentiation, localized to the cytoplasm and main cell processes of iOLs, and downregulated in mature oligodendrocytes both at the transcript and protein levels, thus constituting a novel marker for iOLs, for which we provide an optimal immunofluorescence protocol with a commercial antibody (Sigma, Ct). Second, we show that during remyelination, Tns3 is also expressed in newly formed oligodendrocytes and thus could be used as a hallmark for ongoing remyelination. Third, analyzing both $T n s 3^{\beta g e o}$ gene trap mice and two $\operatorname{Tns} 3^{K O}$ mice, we show that constitutive Tns3 deletion is detrimental for normal development and that the predicted loss of Tns3 full-length transcript and protein is bypassed in the oligodendroglia of surviving homozygous animals, paralleling the intolerance for TNS3 loss-of-function variants found in the human population. Fourth, in vivo CRISPR-mediated Tns3-deletion in neonatal neural stem cells from the subventricular zones leads to a 2 -fold reduction of oligodendrocytes without changes in OPC generation, proliferation, and numbers. Fifth, in vivo Tns3 induced knockout (Tns3-iKO) in postnatal OPCs leads to a 2 -fold reduction of differentiating oligodendrocytes without reducing the overall OPC population, both in grey and white matter brain regions. Finally, we provide evidence, by immunodetection in vivo and video microscopy of primary OPC differentiation cultures, that Tns3-iKO differentiating oligodendroglia upregulate p53, key sensor of cell stress, and present a 4-5-fold increase in apoptosis compared to control oligodendroglia, suggesting that mechanistically Tns3 function is likely required for normal oligodendrocyte differentiation at least in part by mediating integrin- $\beta 1$ survival signaling in differentiating oligodendroglia.

\section{Tns3 is a novel marker for immature oligodendrocytes}

Recent studies has started to uncover genes enriched in immature oligodendrocytes (iOLs), such as Itpr2 (Zeisel et al., 2015; Marques et al., 2016), Enpp6 (Xiao et al., 2016), and Bcas1 (Fard et al., 2017), that could be used as markers for these transient cell populations, particularly interesting to label areas of active (re)myelination in the context of oligodendrocyte and myelin pathology, such as preterm brain injury and multiple sclerosis. Here, we report for the first time that Tns3 is a hallmark of iOLs (figure 2). Tns3 is expressed at high levels in iOLs and downregulated as oligodendrocytes mature into myelinating cells, showing a complete overlap with Itpr2 transcript and protein. We found that a commercial Tns3 antibody (Millipore) also recognizes another nuclear protein that, like Tns3 in the 
cytoplasm, also labels at high levels iOLs, paralleling the case of CC1 antibody, which recognizes both APC and Quaking-7 proteins in oligodendrocytes (Lang et al., 2013; Bin et al., 2016). Upon testing several antibodies, we found one (Sigma Ct) optimally labeling iOLs by immunofluorescence in brain sections and oligodendroglial cell cultures, whereas the Itpr2 commercial antibody we tried did not match this high quality iOLs immunolabeling. The use of BCAS1 antibodies has been proposed to label iOLs (Fard et al., 2017), but has the caveat of being also expressed in mature oligodendrocytes and myelin (Fig. S1E;(Ishimoto et al., 2017). Finally, Enpp6 is very specific for iOLs at the transcript level (Xiao et al., 2016) but to our knowledge, no Enpp6-recognizing antibodies producing good quality immunodetection are yet available. Therefore, Tns3 protein expression in the CNS is a hallmark of iOLs, and the Tns3 Sigma Ct antibody is an optimal reagent to label iOLs during both myelination and remyelination.

\section{Tns3 is required for oligodendroglial differentiation}

Oligodendrocyte differentiation involves substantial generation of new membrane and cell processes composing the 40-60 myelin segments formed by mature oligodendrocytes (Hughes et al., 2018). Actin cytoskeleton remodeling is an important driver of the OL morphological changes undergone during their differentiation (Nawaz et al., 2015; Zuchero et al., 2015). Tensin proteins, linking the extracellular signals received by transmembrane integrins with the actin cytoskeleton in different cell types (Liao and Lo, 2021), are well placed to play an important role in these morphological changes. At the molecular level, it has been shown that the phosphotyrosine-binding domains of tensins interact with the NPXY motifs present in the cytoplasmic tails of integrin- $\beta 1$ in a pTyr-insensitive fashion (Calderwood et al., 2003; Katz et al., 2007; McCleverty et al., 2007), allowing tensins to bring actin filaments, through their actin binding domain, to focal adhesion sites (Liao and Lo, 2021). Given that the extension of OL cell processes' growth cone is guided by the sequential activation of Fyn, FAK and RhoGAP (Thomason et al., 2020), and that high levels of Tns3 protein are detected in the cell body and processes of iOLs coinciding with their large enlargement, Tns3 is thus well placed to mediate integrin signaling to the actin cytoskeleton and play an active role in this large cellular remodeling. Moreover, Integrin- $\beta 1$, FAK/Ptk2, Fyn, p130Cas/Bcar1, and Tns3 are all highly expressed in iOLs (Fig. S13). Here, using three independent approaches, we show that loss of Tns3 in iOLs reduces by half the numbers of oligodendrocytes in the postnatal brain. It is 
therefore very likely that Tns3 act as a mediator of integrin $\alpha 6 \beta 1$ signaling to promote $\mathrm{OL}$ survival and differentiation by mediating actin cytoskeletal remodeling. If so, exogenous activation of integrin $\alpha 6 \beta 1$ in cultured OPCs by $\mathrm{Mn}^{2+}$ (Colognato et al., 2004) would not be expected to increase oligodendrogenesis in Tns3-iKO oligodendroglia. Finally, through its additional ability to bind to EGFR (Cui et al., 2004), whose activation is another driver of oligodendroglial differentiation, Tns3 could also be required for mediation of signaling downstream of growth factor receptor activation in early iOLs; this could also explain the increased death in OLs lacking Tns3.

\section{Tns3 role in immature oligodendrocyte survival}

Programmed cell death regulates developmental oligodendrogenesis, with a large proportion of immature oligodendrocytes degenerating before the fourth week of postnatal life in mice (Barres et al., 1992; Trapp et al., 1997). Also in the adult mouse brain, differentiating OPCs remain in the immature oligodendrocyte stage for roughly 2 days with many of them undergoing programmed cell death (Hughes et al., 2018), indicating that this immature stage is very dependent on survival signals. Apoptotic pathways involving BCL-2 family members have been shown to regulate this oligodendroglial programmed cell death (reviewed in (Hughes and Stockton, 2021). On study has shown that the transcription factor TFEB, involved in autophagy and lysosomal biogenesis, ensures the spatial and temporal specificity of developmental myelination by promoting the expression of ER stress genes and PUMA, a proapoptotic factor inducing Bax-Bak-dependent programmed cell death in differentiating oligodendroglia (Sun et al., 2018). Another recent study showed that during both during homeostasis and remyelination, the activity of the primary sensor of cellular stress, nuclear factor (erythroid-derived 2)-like 2 (Nrf2), induces the expression of Gsta4, a scavenger of lipid peroxidation, with in turn controls apoptosis of immature oligodendrocytes via the mitochondria-associated Fas-Casp8-Bid-axis (Carlström et al., 2020).

Several studies have shown that integrin- $\beta 1$ signaling is required for iOL survival. Neuronalderived signals, including neuregulin and laminin-2, are received by immature oligodendrocytes through integrin- $\beta 1$ signaling that would enhance the function of neuroligin as a survival factor by inducing a survival-dependence switch from the phosphatidylinositol 3kinase-Akt pathway to the mitogen-activated protein kinase (MAPK) pathway, with enhanced MAPK signaling inactivating the pro-apoptotic molecule BAD (Colognato et al., 2002; 
Benninger et al., 2006). Also, PDGF survival signaling in OPCs and myelin formation have been shown to depend on integrin $\alpha 6 \beta 1$ binding to Fyn (Colognato et al, 2004). Tensins typically reside at focal adhesions, which connect the extracellular matrix (ECM) to the cytoskeletal networks through integrins and their associated protein complexes (Kumar, 1998; Liao and Lo, 2021), with focal adhesions mediating both outside-in and inside-out signaling pathways that regulate cellular events, such as cell attachment, migration, proliferation, apoptosis, and differentiation (Liao and Lo, 2021). In this study, we show that Tns3 expression timing during oligodendrogenesis, parallels that of integrin- $\beta 1$, and we provided evidence of Tns3 and integrin- $\beta 1$ co-localization in dotted structures resembling nascent and focal adhesion in the cytoplasm and processes of immature oligodendrocytes. Therefore, given the similar apoptotic phenotypes found in differentiating oligodendroglia upon integrin- $\beta 1$ (Benninger et al., 2006) or Tns3 oligodedendroglial specific knockout (this study), we suggest that Tns3 is required for integrin- $\beta 1$ survival signal in immature oligodendrocytes. Moreover, we suggest that this would lead to cellular stress of Tns3-deleted differentiating oligogodendroglia and to the upregulation of p53, master regulator of cellular stress and apoptosis, which has been previously been shown to be involved in the apoptosis of human oligodendrocytes in the context of MS (Ladiwala et al., 1999; Wosik et al., 2003) and in the cuprizone demyelination mouse model (Li et al., 2008; Luo et al., 2021).

In summary, here we have generated powerful genetic tools allowing to assess for the first time the role of Tns3 in the CNS, shown that Tns3 protein is found at high levels in the cytoplasm and main processes of immature oligodendrocytes thus constituting a new marker of this oligodendroglial stage, and demonstrated by different genetic approaches that Tns3deletion leads to a two-fold reduction in differentiating oligodendrocytes, explained at least in part by their increased apoptosis due to $p 53$ upregulation and likely the loss of integrin- $\beta 1$ mediated survival signaling. Follow up studies using these tools should unravel with more detail the molecular mechanisms mediated by Tns3 not only in immature oligodendrocytes during developmental myelination but also in pathological contexts such as preterm birth dysmyelination, adult demyelination in MS and glioblastoma, this last recently associated with reduced levels of Tns3 (Chen et al., 2017). 


\section{MATERIAL \& METHODS}

\section{Animals}

All animal procedures were performed according to the guidelines and regulations of the Inserm ethical committees (authorization \#A75-13-19) and animal experimentation license A75-17-72 (C.P.). Both males and females were included in the study. Mice were maintained in standard conditions with food and water ad-libitum in the ICM animal facilities. Tensin3 gene trap mouse line (Tns3 $\left.{ }^{\beta g e o}\right)$ was from Su Hao Lo lab (UC Davis, USA). Mice used for ChIPseq analysis were wild type Swiss obtained from Janvier Labs. Tns $3^{\text {flox }}$ were crossed with Pdgfra-CreER ${ }^{T}$ (Kang et al., 2010) and Rosa26 stop-floxed-YFP $^{\text {mice }}$ to generate Tns $3^{\text {flox; }}$ PdgfraCreER ${ }^{T}$; Rosa26 $6^{\text {stop-floxed-YFP }}$ mice line. Pdgfra-CreER $;$ Rosa26 stop-floxed-YFP mice were used as controls.

\section{Development of CRISPR/Cas9 tools}

CRISPOR software (http://crispor.tefor.net/) was used to design gRNAs with predicted cutting efficiency and minimal off-target and PCR amplification primers. The validation of Tns3targeting CRISPR/Cas9 system was performed in 3T3 cells by transfection with Lipofectamine 3000 of PX459 plasmids containing 4 different sgRNA sequences. After 2 days incubation, puromycin was added to medium for 4 days allowing survival of cells containing the $P X 459$ plasmid. Three days after proliferation in fresh medium without puromycin, DNA was extracted using DNeasy blood \& tissue kit (Qiagen). The target DNA for 5' Tns3 region was amplified by PCR using primers with the following sequences: Forward: 5'-AGG TGG CCT TCA GCT CAGT-3', Reverse: 5'-GCT ATC ATC CCC ACT CAC CA-3'; annealing temperature of $64^{\circ} \mathrm{C}$, with the PCR product expected to be 326bp. DNA from 3' Tns3 target region was amplified using primer with the following sequences: Forward: 5'-CCA GTC AGT GGT GAC ATT GTTT-3', Reverse: 5'-ACT GTT CCC AGG TTG CTA TCAT-3'), annealing temperature of $58^{\circ} \mathrm{C}$, with the PCR product expected to be $419 \mathrm{bp}$.

Cutting efficiency of sgRNA was verified by T7 endonuclease I, following the beta protocol of IDTE synthetic biology for amplification of genomic DNA and detecting mutations, using PAGE. In order to generate plasmids that will insert CRISPR tools into the genome of the transfected cells and lead to permanent expression of the targeting tools, the PX458 (GFP) or 
PX459 (Puromycin) plasmids were subcloned into a Tol2-containing sequence backbone (obtained from Tol2-mCherry expressing plasmid kindly provided by Jean Livet, Institut de la Vision, Paris).

\section{Generation of Tns3 $3^{\text {Tns3-V5 }}$ knock-in mice}

Tns $3^{V 5}$ mice were generated at the Curie Institute mouse facility. Briefly, the Cas 9 protein, the crRNA, the tracrRNA and a ssODN targeting vector for the Tns3 gene had been microinjected into a mouse egg cell, which was transplanted into a C57BL/6J-BALB/cJ female surrogate. Pups presenting HDR insertion of the V5 tag were selected after genotyping.

\section{Generation of $T n s 3^{4 d e l}$ and $T n s 3^{14 d e l}$ knockout mice}

Tns $3^{K O}$ mice were generated at the ICM mice facility. Briefly, the Cas9 protein, the crRNA, the tracrRNA and a targeting vector for the Tns3 gene had been microinjected into a mouse egg cell transplanted into a C57BL/6J female surrogate. Pups with NHEJ mutations inducing a gene frameshift were selected after genotyping and Sanger sequencing verification. Finally, only two lines containing indels of 4 and 14 nucleotide deletions were maintained and studied.

\section{Generation of $T n s 3^{\text {Flox }}$ mice and tamoxifen administration}

Tns3 conditional knockout mice (LoxP-Exon9-LoxP) were generated at the Transgenic Core Facility of the University of Copenhagen. The repair template contained homology arms of $771 \mathrm{bp}$ and $759 \mathrm{bp}$ length and loxP sequences flanking Exon 9, and was synthesized by Invitrogen and verified by Sanger sequencing. Two gRNAs were designed at the Transgenic Core Facility that target a DNA sequence in the proximity of each loxP site. The gRNAs were designed in a fashion where the insertion of the loxP disrupts the targeting site, thus preventing retargeting of the repaired DNA. mES (mouse embryonic stem cell) method was used for the generation of this mouse model by transfecting ES cells with the repair construct (dsDNA) together with two plasmids - each containing each gRNA. Identification of the positive mES clones was done via a combination of a PCR genotyping and Sanger sequencing confirmation.

Tamoxifen (Sigma, T5648) was dissolved in corn oil (Sigma, C-8267) and injected subcutaneously at $20 \mathrm{mg} / \mathrm{ml}$ concentration at P7 (30 $\mathrm{\mu l})$ in $\mathrm{Ctrl}$ and Tns3-iKO animals. Brains were then collected at P21. 


\section{Postnatal electroporation}

Postnatal brain electroporation (Boutin et al., 2008) was adapted to target the dorsal SVZ. Briefly, postnatal day 2 (P2) pups were cryoanesthetized for 2 min on ice and $1.5 \mu$ of plasmid was injected into their left ventricle using a glass capillary. Plasmids were injected at a concentration of $2-2.5 \mu \mathrm{g} / \mu \mathrm{l}$. Electrodes (Nepagene CUY650P10) coated with highly conductive gel (Signagel, signa250) were positioned in the dorso-ventral axis with the positive pole dorsal. Five electric pulses of $100 \mathrm{~V}, 50 \mathrm{~ms}$ pulse $\mathrm{ON}, 850 \mathrm{~ms}$ pulse OFF were applied using a Nepagene CUY21-SC electroporator. Pups were immediately warmed up in a heating chamber and brought to their cages at the end of the experiment.

\section{Demyelinating lesions}

Before surgery, adult (2-3months) WT mice were weighed and an analgesic (buprenorphine, $30 \mathrm{mg} / \mathrm{g}$ ) was administered to prevent postsurgical pain. The mice were anesthetized by anesthetized by induction of isoflurane (ISO-VET). Ocrygel (TVm) was put on their eye to prevent dryness and lidocaine in cream (Anesderm 5\%) was put on the ear bars to prevent pain. After cutting of the skin, a few drop of liquid lidocaine were put to prevent pain. Focal demyelinating lesions were induced by stereotaxic injection of $1 \mu \mathrm{l}$ of lysolecithin solution (LPC, Sigma, $1 \%$ in $0.9 \% \mathrm{NaCl}$ ) into the corpus callosum (CC; at coordinates: $1 \mathrm{~mm}$ lateral, $1.3 \mathrm{~mm}$ rostral to bregma, $1.7 \mathrm{~mm}$ deep) using a glass-capillary connected to a $10 \mu \mathrm{l}$ Hamilton syringe. Animals were left to recover in a warm chamber before being returned into their housing cages.

\section{Tissue processing}

Postnatal mouse were transcardially perfused with $15 \mathrm{ml}$ (P14) or $25 \mathrm{ml}$ (>P21) of $2 \%$ PFA freshly prepared from 32\% PFA solution (Electron Microscopy Sciences, 50-980-495). Perfused brains were dissected out, dehydrated in $10 \%$ sucrose followed by $20 \%$ sucrose overnight, and embedded in OCT (BDH) before freezing and sectioning (16 $\mu \mathrm{m}$ thickness) in a sagittal plane with a cryostat microtome (Leica). 


\section{Magnetic Assisted Cell Sorting (MACS)}

Dissociation of cortex and corpus callosum from mice brain was done using neural tissue dissociation kit (P) (Miltenyi Biotec; ref 130-093-231). Briefly, cortices were dissected from P7, P12, P14 or P21 mice and dissociated using a MACS dissociator (Miltenyi Biotec; ref 130096-427) followed by filtration through a $70 \mu \mathrm{m}$ cell strainer (Smartstainer; Miltenyi Biotec; ref 130-098-462). Myelin residues were eliminated from P12, P14 and P21 mice cortices during an additional step using the debris removal kit (Miltenyi Biotec; ref 130-090-101). Cells were suspended in a $0.5 \%$ NGS solution then incubated with anti-PDGFR $\alpha$ or anti-O4 coupled-beads (Miltenyi Biotec; ref 130-094-543 and 130-096-670). Unbound bead-coupled antibodies were washed away by centrifugation, leaving bound cells which were sorted using MultiMACS Cell24 Separator Plus (Miltenyi Biotec; ref 130-098-637). Sorted cells were either plated in culture plates for in vitro cell study or centrifuged at $1200 \mathrm{rpm}$ and used for Western blot analysis or ChIP-seq.

\section{Immunofluorescence staining and microscopy}

Postnatal mouse brain cryosections were dried for 20 minutes at room temperature, before adding the blocking solution (10\% normal goat serum (NGS, Eurobio, CAECHVOO-OU) and $0.1 \%$ Triton X-100 in PBS) for one hour at room temperature. Primary antibodies were diluted (dilutions indicated in Table1) in the same blocking solution and incubated on the slices overnight at $4^{\circ} \mathrm{C}$. After washing with $0.05 \%$ Triton X-100 in PBS, sections were incubated with secondary antibodies conjugated to AlexaFluor-488, AlexaFluor-594 and AlexaFluor-647 (Thermo, 1:1,000). Finally, cell nuclei were labeled with DAPI (1/10000, Sigma-Aldrich ${ }^{\circledR}$, D9542-10MG), and slices are mounted in Fluoromount-G ${ }^{\circledR}$ (SouthernBiotech, Inc. 15586276).

In Situ Cell Death detection kit (Roche, 12156792910) was used to do Tunel experiment on P21 mouse brains. Briefly, tissues were processed as mentioned above with anti-GFP and anti-CC1 and fixed in fixation solution for $20 \mathrm{~min}$ at room temperature. After washing, slices were permeabilized for $2 \mathrm{~min}$ in permeabilisation solution $(0.1 \%$ Triton $\mathrm{X}-100 ; 0.1 \%$ sodium citrate) and TUNEL reaction mixture was put on samples for one hour at $37^{\circ} \mathrm{C}$. Tissues were then mounted in Fluoromount-G ${ }^{\circledR}$.

Fixed coverslips were blocked in blocking solution (10\% normal goat serum (NGS, Eurobio, CAECHVOO-OU) and 0.1\% Triton X-100 in PBS) for 30 minutes at room temperature, incubated in the primary antibodies for 45 minutes at room temperature and washed 3 times 
in 1x PBS. Secondary antibodies were applied for 45 minutes at room temperature and washed 3 times in 1X PBS. Coverslips were then incubated with DAPI solution for 5 minutes at RT. A final washing was done before mounting the coverslips on slides to be visualized under the microscope.

Immunofluorescence was visualized with Zeiss ${ }^{\circledR}$ Axio Imager.M2 microscope with Zeiss $^{\circledR}$ Apotome system. Pictures were taken as stacks of $5-10 \mu \mathrm{m}$ with $0.5 \mu \mathrm{m}$ between sections. Image acquisition and processing are achieved by ZEN Microscopy and imaging Software, Z-projections and orthogonal projections were done in ImageJ and processed with Adobe Photoshop. Figures were made using Adobe Illustrator.

\section{Western blot}

Proteins from MACsorted cells were extracted during 30 minutes at $4^{\circ} \mathrm{C}$ in RIPA buffer (ThermoFisher; $50 \mu \mathrm{L}$ per million cells, 89901) supplemented with Halt ${ }^{\mathrm{TM}}$ Protease Inhibitor Cocktail (100X; ThermoFisher, 87786). Protein concentration in the supernatant was estimated using the Pierce Detergent Compatible Bradford Assay Kit (Thermofisher, 23246). For each Western Blot, we used $50 \mu \mathrm{g}$ of proteins denaturated for 10 minutes at $95^{\circ} \mathrm{C}$ with added $\beta$-mercaptoethanol (from $24 X$ stock) and BoltTM LDS Sample Buffer (4X) (ThermoFisher, B0007). Sodium dodecyl sulfate-polyacrylamide gel electrophoresis (SDSPAGE) was performed using precast 4-12\% polyacrylamide gradient gels (ThermoFisher, NW04122BOX), submerged at $4^{\circ} \mathrm{C}$ in Bolt ${ }^{\mathrm{TM}}$ MOPS SDS Running Buffer (ThermoFisher, B0001) using Mini Gel Tank and Blot Module Set (ThermoFisher, NW2000). Precision Plus Protein ${ }^{\mathrm{TM}}$ All Blue protein standards (BioRad, 1610373EDU) were run alongside the samples as a protein migration control. Proteins were separated for 90 minutes at $90 \mathrm{~V}$, after which gels were transferred onto Amersham Protran $0.2 \mu \mathrm{m}$ nitrocellulose membrane (Dutscher, 10600001) immersed at $4^{\circ} \mathrm{C}$ in NuPAGE Transfer Buffer (ThermoFisher, NP0006-1) for 90 minutes at 60V. Following transfer, membranes were incubated for $1 \mathrm{~h}$ in TBS-T, $10 \%$ dry milk to aid blocking of non-specific binding by the antibodies. Primary antibodies diluted in TBS-T were incubated with the membrane overnight at $4^{\circ} \mathrm{C}$ with shaking. After three washes in TBS-T, membranes were incubated with HRP-conjugated secondary antibodies diluted in TBS-T for $1 \mathrm{~h}$ at $4^{\circ} \mathrm{C}$ with shaking, then developed using Pierce ${ }^{\mathrm{TM}}$ ECL Western Blotting Substrate (ThermoFisher, 32109) 
and imaged with the ChemiDoc ${ }^{T M}$ Touch Imaging System (BioRad, 1708370). Western blot detection of actin was performed as loading control.

\section{Videomicroscopy}

Tamoxifen was administered to P5 Tns3 ${ }^{\text {flox; }}$ Pdgfro-CreER ; Rosa26 $6^{\text {stop-floxed-YFP }}$ and Tns3 $3^{\text {flox; }}$ Pdgfra-WT; Rosa26 $6^{\text {stop-floxed-YFP }}$ littermates. Brains were dissected out at P7 in order to MACSort OPCs using an anti-PDGFR $\alpha$ antibody coupled to magnetic beads. OPCs were plated in poly-L-ornithine (sigma, P4957) coated $\mu$-Slide 8 Well Glass Bottom slide (Ibidi, 80827) at 40,000 cells/mm2 in OPC proliferative medium : DMEM/F12 (life technology, 31331028), 5mM

HEPES buffer (life technology, 15630056), 0.6\% glucose (Sigma, G8769), 1x penicillin/streptomycin (life technology, 15140122), N2 supplement (life technology, 17502048), B27 supplement (life technology, 17504044), 20ng/ $\mu$ I EGF (Peprotech, AF-100-15),

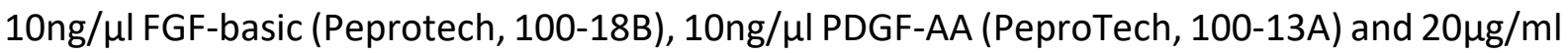
Insulin (Sigma, 16634). After 3 days of proliferation, medium was replaced by growth factor depleted medium. Cell differentiation was tracked for 3 days using time-lapse video recording. Cells were put in to a videomicroscope (Zeiss AxioObserver 7, provided by ICM-quant and CELIS facilities) with a humidified incubator at $37^{\circ} \mathrm{C}$ with a constant $5 \% \mathrm{CO} 2$ supply. Images for both FITC and bright field were acquired every 10 minutes.

\section{Chromatin immunoprecipitation (ChIP)}

ChIP-seq assays were performed as described previously (Marie et al; 2018), using iDeal ChIPseq kit for Transcription Factors (Diagenode, C01010055). Briefly, $\mathrm{O4}^{+} \mathrm{MACS}$ (1) fixed in $1 \%$ formaldehyde (EMS, 15714) for $10 \mathrm{~min}$ at room temperature and the reaction was quenched with $125 \mathrm{mM}$ glycine for $5 \mathrm{~min}$ at room temperature. Lysates were sonicated with a Bioruptor Pico sonicator (Diagenode, total time $8 \mathrm{~min}$ ) and $4 \mu \mathrm{g}$ of antibodies were added to sheared chromatin (from 4 million cells for Olig2 and from 1 million cells for histone marks) and incubated at $4^{\circ} \mathrm{C}$ overnight on 10rpm rotation. Antibodies used were: mouse anti-Olig2 antibody (Millipore, MABN50), rabbit anti-H3K4me3 antibody (Active motif, 39060), rabbit anti-H3K27Ac antibody (Active motif, 39034), rabbit anti-H3K4me1 antibody (Ozyme, 5326T), mouse anti-H3K27me3 antibody (Abcam, ab6002). Mock (Rabbit IgG) was used as negative control. Chromatin-protein complexes were immunoprecipitated with protein A/G magnetic beads and washed sequentially according to the manufacturer (Diagenode, C01010055). DNA 
fragments were then purified using IPure beads v2 (Diagenode, C01010055). Input (nonimmunoprecipitated chromatin) was used as control in each individual experiment.

The ChIP-seq libraries were prepared using ILLUMINA Truseq ChIP preparation kit and sequenced with ILLUMINA Nextseq 500 platform.

\section{ChIP-seq analysis}

All ChIP-seq analysis were done using the Galaxy Project (https://usegalaxy.org/). Reads were trimmed using Cutadapt (--max-n 4) and Trimmomatic (TRAILING 1; SLIDINGWINDOW 4 and cutoff 20; LEADING 20; MINLEN 50), and mapped using Bowtie2 onto mm10 mouse reference genome (-X 600; -k 2; --sensitive). PCR-derived duplicates were removed using PICARD MarkDuplicates. Bigwig files were generated with bamCoverage (binsize=1). Peak calling was performed using MACS2 callpeak with Input as control and with options: --qvalue 0.05; -nomodel; --keep-dup 1; --broad (only for histone marks). Blacklisted regions were then removed using bedtools Intersect intervals.

Visualization of coverage and peaks was done using IGV ((Robinson et al., 2011); http://software.broadinstitute.org/software/igv/home). Intersection and analysis of bound genes were done using Genomatix (https://www.genomatix.de/). Chd7, Chd8 and Mock ChIPseq datasets are from (Marie et al., 2018). Heatmap was done using R (4.0) using pheatmap package. GO analysis was done using Enrichr GO Biological Process 2021.

Two replicates were done for Olig2, with one of them of better quality $(53,960$ peaks for replicate 1 and 14,242 peaks for replicate 2). Only the peaks found in both replicates $(6,781)$ and the peaks from replicate 1 which were found in regulatory elements (13948) were considered (16578 in total). Three replicates were done for H3K4me3, two replicates were done for H3K27me3 and one replicate was done for H3K27Ac and H3K4me1. Intersection of these datasets was done using bedtools Intersect intervals.

Peaks overlapping with regions between 1000bp upstream of transcription start site (TSS) and 10bp downstream of TSS were identified as "promoters" (Genomatix). "Active promoters" were represented by peaks for H3K4me3 and H3K27Ac. "Repressed promoters" were represented by peaks for H3K27me3 and no active marks. "Poised promoters" were represented by peaks for $\mathrm{H} 3 \mathrm{~K} 4 \mathrm{me} 1$ and no active or repressed mark. Regions outside promoters containing histone marks were considered as "enhancers". "Active enhancers" 
were represented by peaks for H3K27Ac. "Repressed enhancers" were represented by peaks for H3K27me3 and no active marks. "Poised enhancers" were represented by peaks for H3K4me1 and no active or repressed mark. Genes were considered associated if the peaks were present in the promoter or within a range of $100 \mathrm{~kb}$ from the middle of the promoter and the gene expression was medium to high ("active"), low ("poised") or not ("repressed") expressed (based on control RNA-seq dataset in GSE116601).

\section{Analysis of RNAseq}

Raw data were downloaded from GEO datasets GSE107919 and GSE116601 and processed throught the Galaxy Project (https://usegalaxy.org/) using RNAstar for alignment on mm10 reference genome and featureCounts to obtain counts. CPM (count per million), FPKM and statistical analysis were done with $\mathrm{R}(4.0)$ using edgeR package.

Using control RNA-seq dataset in GSE116601, genes were classified as not (below first quartile), low (between first quartile and mean), medium (between mean and third quartile) and high (above third quartile).

\section{Analysis of scRNAseq}

Counts per gene were downloaded from GEO datasets GSE75330 and GSE95194, and processed in R (4.0) using the following packages: Seurat (3.0) for data processing, sctransform for normalization, and ggplot2 for graphical plots. Seurat objects were first generated for each dataset independently using CreateSeuratObject function (min.cells = 5, min.features $=100$ ). Cell neighbors and clusters were found using FindNeighbors (dims = 1:30) and FindClusters (resolution $=0.4$ ) functions. Clusters were manually annotated based on the top 50 markers obtained by the FindAllMarkers, adopting mainly the nomenclature from Marques 2016. Using the subset function, we selected only the clusters containing neural progenitors and oligodendroglia cells. Using the merge function, we combined both oligodendroglial datasets into a single Seurat object (OLgliaDevP) containing 5516 cells. The new object was subjected to NormalizeData, FindVariableFeatures, ScaleData, RunPCA, and RunUMAP functions with default parameters. Different OPC clusters were fused into a single one keeping apart the cycling OPC cluster. For DimPlots and Dotplots, clusters were ordered 
by stages of oligodendrogenesis from neural stem cells (NSCs) to myelinating OLs. R script is provided as supplementary file.

\section{Data Resources}

Raw data files have been deposited in the NCBI Gene Expression Omnibus under accession number GEO: XX

\section{Contact for Reagent and Resource Sharing}

Further information and requests for reagents may be directed to, and will be fulfilled by the corresponding author Carlos Parras (carlos.parras@icm-institute.org).

\section{ACKNOWLEDGMENTS}

We thank D. Bergles for the PDGFR $\alpha:$ :CreER ${ }^{T}$ mice. All animal work was conducted at the ICM PHENOPARC Core Facility. Data generated relied on ICM Core Facilities: PHENO ICMice, iGenSeq, iVector, CELIS, Histomics, and ICM Quant, and we thank all personnel involved for their contribution and help. The Core Facilities were supported by the "Investissements d'avenir" (ANR-10- IAIHU-06 and ANR-11-INBS-0011-NeurATRIS) and the "Fondation pour la Recherche Médicale". This work was supported by funding by grants from the National Multiple Sclerosis Society (NMSS RG-1501-02851), and the Fondation pour l'Aide à la Recherche sur la Sclérose en Plaques (ARSEP 2014, 2015, 2018, 2019, 2020). E.M, H.H, and C.M. were supported by funding from Sorbonne Université. C.M. was also supported by Fondation pour la Recherche Médicale (FRM, FDT20160435662) and ARSEP grant 2018-2020. 


\section{Table 1}

\begin{tabular}{|c|c|c|c|}
\hline \multicolumn{4}{|l|}{ Antibodies } \\
\hline Rabbit anti Tensin 1 & Su Hao Lo, UC Davis & None & 1:100 \\
\hline Rabbit anti Tensin2 & Su Hao Lo, UC Davis & None & 1:100 \\
\hline Rabbit anti Tensin3 & Sassan Hafizi, University of Portsmouth & None & $1: 1000$ \\
\hline Rabbit anti Tensin3 & Millipore & AB229 & $1: 500$ \\
\hline Rabbit anti Tensin3 & Thermo-Fisher & PA5-116022 & $1: 1000$ \\
\hline Mouse monoclonal anti Tensin3 & Santa Cruz Biotech & sc-376367 & $1: 500$ \\
\hline Rabbit anti Tns3 (C-terminal) & Sigma-Aldrich & SAB4200205 & $1: 200$ \\
\hline Rabbit anti Tns3 (TN-17) & Sigma-Aldrich & SAB4200416 & $1: 400$ \\
\hline Rabbit anti V5 tag & Millipore & AB3792 & $1: 2000$ \\
\hline Mouse monoclonal anti V5 tag & Invitrogen & R960-25 & $1: 1000$ \\
\hline Rat anti PDGFR $\alpha$ & BD Biosciences & 558774 & 1:250 \\
\hline mouse monoclonal anti Olig1 & NeuroMab & $75-180$ & $1: 1000$ \\
\hline Mouse monoclonal anti Olig2 & Millipore & MABN50 & $1: 500$ \\
\hline Mouse monoclonal anti CNPase & Millipore & MAB326R & $1: 250$ \\
\hline Rabbit anti IP3 receptor 2 (Itpr2) & Millipore & AB3000 & $1: 40$ \\
\hline mouse polyclonal anti Nkx2.2 & Developmental Studies Hybridoma Bank & None & $1: 4$ \\
\hline $\begin{array}{l}\text { mouse monoclonal anti CC1 } \\
\text { (Quaking 7) }\end{array}$ & Calbiochem & OP80 & $1: 100$ \\
\hline mouse monoclonal anti MOG & ICM, Paris Hybridoma & AA3 & $1: 20$ \\
\hline Mouse monoclonal anti Opalin & Santa Cruz Biotech & sc-374490 & $1: 500$ \\
\hline Chicken polyclonal anti GFP & Aves Labs & GFP-1020 & $1: 1000$ \\
\hline Rabbit anti-GFP & Life Technologies & A6455 & $1: 1000$ \\
\hline Mouse anti MCM2 (BM28) & BD Biosciences & 610701 & $1: 500$ \\
\hline Rabbit anti-p53 & Leica & P53-CM5P-L & $1: 500$ \\
\hline Rat anti F4/80 & Abd Serotec & MCA497 & $1: 100$ \\
\hline Integrin- $\beta 1$ & Proteintech & 12594-1-AP & $1: 100$ \\
\hline $\begin{array}{l}\text { Mouse monoclonal anti beta- } \\
\text { galactosidase }\end{array}$ & Promega & Z3783 & $1: 1000$ \\
\hline Chicken anti-GFAP & Aveslabs & $75-240$ & $1: 1000$ \\
\hline HRP-conjugated anti Rabbit & Biorad & 1706515 & $1: 5000$ \\
\hline HRP-conjugated anti Mouse & Sigma-Aldrich & NA931-1ML & $1: 5000$ \\
\hline HRP-conjugated anti Rat & Thermo Fisher Scientific & A10549 & $1: 5000$ \\
\hline rabbit anti-H3K4me3 & Active motif & 39060 & \\
\hline rabbit anti-H3K27Ac & Active motif & 39034 & \\
\hline mouse anti-H3K27me3 & Abcam & ab6002 & \\
\hline rabbit anti-H3K4me1 & Ozyme & $5326 \mathrm{~T}$ & \\
\hline
\end{tabular}


bioRxiv preprint doi: https://doi.org/10.1101/2022.02.25.481980; this version posted February 27, 2022. The copyright holder for this preprint (which was not certified by peer review) is the author/funder, who has granted bioRxiv a license to display the preprint in perpetuity. It is made available under aCC-BY-NC-ND 4.0 International license.

\section{REFERENCES}

Aubrey BJ, Kelly GL, Janic A, Herold MJ, Strasser A (2018) How does p53 induce apoptosis and how does this relate to p53mediated tumour suppression? Cell Death \& Differentiation 25:104-113.

Barres BA, Hart IK, Coles HS, Burne JF, Voyvodic JT, Richardson WD, Raff MC (1992) Cell death and control of cell survival in the oligodendrocyte lineage. Cell 70:31-46.

Benninger $\mathrm{Y}$, Colognato $\mathrm{H}$, Thurnherr T, Franklin RJM, Leone DP, Atanasoski S, Nave K-A, ffrench-Constant C, Suter U, Relvas JB (2006) B1-Integrin Signaling Mediates Premyelinating Oligodendrocyte Survival But Is Not Required for CNS Myelination and Remyelination. The Journal of Neuroscience 26:7665-7673.

Bergles DE, Richardson WD (2016) Oligodendrocyte Development and Plasticity. Cold Spring Harbor Perspectives in Biology 8.

Bin JM, Harris SN, Kennedy TE (2016) The oligodendrocyte-specific antibody 'CC1' binds Quaking 7. Journal of Neurochemistry 139:181-186

Boutin C, Diestel S, Desoeuvre Al, Tiveron M-C, Cremer H (2008) Efficient In Vivo Electroporation of the Postnatal Rodent Forebrain. PLoS ONE 3:e1883.

Bryois J et al. (2020) Genetic identification of cell types underlying brain complex traits yields insights into the etiology of Parkinson's disease. Nature Genetics 52:482-493.

Cao X, Kaneko T, Li JS, Liu A-D, Voss C, Li SSC (2015) A phosphorylation switch controls the spatiotemporal activation of Rho GTPases in directional cell migration. Nature communications 6:7721.

Carlström KE, Zhu K, Ewing E, Krabbendam IE, Harris RA, Falcão AM, Jagodic M, Castelo-Branco G, Piehl F (2020) Gsta4 controls apoptosis of differentiating adult oligodendrocytes during homeostasis and remyelination via the mitochondriaassociated Fas-Casp8-Bid-axis. Nature communications 11:4071.

Castelijns B, Baak ML, Timpanaro IS, Wiggers CRM, Vermunt MW, Shang P, Kondova I, Geeven G, Bianchi V, de Laat W, Geijsen N, Creyghton MP (2020) Hominin-specific regulatory elements selectively emerged in oligodendrocytes and are disrupted in autism patients. Nature communications 11:301.

Chamling X, Kallman A, Fang W, Berlinicke CA, Mertz JL, Devkota P, Pantoja IEM, Smith MD, Ji Z, Chang C, Kaushik A, Chen L, Whartenby KA, Calabresi PA, Mao H-Q, Ji H, Wang T-H, Zack DJ (2021) Single-cell transcriptomic reveals molecular diversity and developmental heterogeneity of human stem cell-derived oligodendrocyte lineage cells. Nature communications 12:652.

Chang A, Tourtellotte WW, Rudick R, Trapp BD (2002) Premyelinating oligodendrocytes in chronic lesions of multiple sclerosis. N Engl J Med 346:165-173.

Chen H-Y, Lin L-T, Wang M-L, Laurent B, Hsu C-H, Pan C-M, Jiang W-R, Chen P-Y, Ma H-I, Chen Y-W, Huang P-I, Chiou A, Chiou S-H (2017) Musashi-1 Enhances Glioblastoma Cell Migration and Cytoskeletal Dynamics through Translational Inhibition of Tensin3. Scientific Reports 7:8710.

Chiang M-K, Liao Y-C, Kuwabara Y, Lo SH (2005) Inactivation of tensin3 in mice results in growth retardation and postnatal lethality. Developmental Biology 279:368-377.

Colognato H, Ramachandrappa S, Olsen IM, ffrench-Constant C (2004) Integrins direct Src family kinases to regulate distinct phases of oligodendrocyte development. J Cell Biol 167:365-375.

Colognato H, Baron W, Avellana-Adalid V, Relvas JB, Evercooren AB-V, Georges-Labouesse E, ffrench-Constant C (2002) CNS integrins switch growth factor signalling to promote target-dependent survival. Nat Cell Biol 4:833-841.

Compston A, Coles A (2002) Multiple sclerosis. The Lancet 359:1221-1231.

Cui Y, Liao YC, Lo SH (2004) Epidermal growth factor modulates tyrosine phosphorylation of a novel tensin family member, tensin3. Molecular cancer research : MCR 2:225-232.

Elsesser O, Fröb F, Küspert M, Tamm ER, Fujii T, Fukunaga R, Wegner M (2019) Chromatin remodeler Ep400 ensures oligodendrocyte survival and is required for myelination in the vertebrate central nervous system. Nucleic acids research 47:6208-6224.

Emery B, Lu QR (2015) Transcriptional and Epigenetic Regulation of Oligodendrocyte Development and Myelination in the Central Nervous System. Cold Spring Harbor Perspectives in Biology 7. 
bioRxiv preprint doi: https://doi.org/10.1101/2022.02.25.481980; this version posted February $27,2022$. The copyright holder for this preprint (which was not certified by peer review) is the author/funder, who has granted bioRxiv a license to display the preprint in perpetuity. It is made available under aCC-BY-NC-ND 4.0 International license.

Fard MK, van der Meer F, Sánchez P, Cantuti-Castelvetri L, Mandad S, Jäkel S, Fornasiero EF, Schmitt S, Ehrlich M, Starost L, Kuhlmann T, Sergiou C, Schultz V, Wrzos C, Brück W, Urlaub H, Dimou L, Stadelmann C, Simons M (2017) BCAS1 expression defines a population of early myelinating oligodendrocytes in multiple sclerosis lesions. Science Translational Medicine 9:eaam7816.

Ffrench-Constant C, Raff MC (1986) The oligodendrocyte-type-2 astrocyte cell lineage is specialized for myelination. Nature 323:335-338.

Funfschilling U, Supplie LM, Mahad D, Boretius S, Saab AS, Edgar J, Brinkmann BG, Kassmann CM, Tzvetanova ID, Mobius W, Diaz F, Meijer D, Suter U, Hamprecht B, Sereda MW, Moraes CT, Frahm J, Goebbels S, Nave K-A (2012) Glycolytic oligodendrocytes maintain myelin and long-term axonal integrity. Nature 485:517-521.

Georgiadou M, Lilja J, Jacquemet G, Guzmán C, Rafaeva M, Alibert C, Yan Y, Sahgal P, Lerche M, Manneville J-B, Mäkelä TP, Ivaska J (2017) AMPK negatively regulates tensin-dependent integrin activity. Journal of Cell Biology 216:1107-1121.

Grubman A, Chew G, Ouyang JF, Sun G, Choo XY, McLean C, Simmons RK, Buckberry S, Vargas-Landin DB, Poppe D, Pflueger J, Lister R, Rackham OJL, Petretto E, Polo JM (2019) A single-cell atlas of entorhinal cortex from individuals with Alzheimer's disease reveals cell-type-specific gene expression regulation. Nature Neuroscience 22:2087-2097.

He D, Marie C, Zhao C, Kim B, Wang J, Deng Y, Clavairoly A, Frah M, Wang H, He X, Hmidan H, Jones BV, Witte D, Zalc B, Zhou $X$, Choo DI, Martin DM, Parras C, Lu QR (2016) Chd7 cooperates with Sox10 and regulates the onset of CNS myelination and remyelination. Nat Neurosci advance online publication.

Hughes EG, Stockton ME (2021) Premyelinating Oligodendrocytes: Mechanisms Underlying Cell Survival and Integration. Frontiers in Cell and Developmental Biology 9.

Hughes EG, Orthmann-Murphy JL, Langseth AJ, Bergles DE (2018) Myelin remodeling through experience-dependent oligodendrogenesis in the adult somatosensory cortex. Nature Neuroscience 21:696-706.

Ishimoto T, Ninomiya K, Inoue R, Koike M, Uchiyama Y, Mori H (2017) Mice lacking BCAS1, a novel myelin-associated protein, display hypomyelination, schizophrenia-like abnormal behaviors, and upregulation of inflammatory genes in the brain. Glia 65:727-739.

Kang SH, Fukaya M, Yang JK, Rothstein JD, Bergles DE (2010) NG2+ CNS Glial Progenitors Remain Committed to the Oligodendrocyte Lineage in Postnatal Life and following Neurodegeneration. Neuron 68:668-681.

Kastenhuber ER, Lowe SW (2017) Putting p53 in Context. Cell 170:1062-1078.

Katz M et al. (2007) A reciprocal tensin-3-cten switch mediates EGF-driven mammary cell migration. Nat Cell Biol 9:961-969.

Kessaris N, Fogarty M, lannarelli P, Grist M, Wegner M, Richardson WD (2006) Competing waves of oligodendrocytes in the forebrain and postnatal elimination of an embryonic lineage. Nat Neurosci 9:173-179.

Kumar CC (1998) Signaling by integrin receptors. Oncogene 17:1365-1373.

Küspert M, Wegner M (2016) SomethiNG 2 talk about-Transcriptional regulation in embryonic and adult oligodendrocyte precursors. Brain Research 1638, Part B:167-182.

Ladiwala U, Li H, Antel JP, Nalbantoglu J (1999) p53 Induction by Tumor Necrosis Factor- $\alpha$ and Involvement of p53 in Cell Death of Human Oligodendrocytes. Journal of Neurochemistry 73:605-611.

Lang J, Maeda Y, Bannerman P, Xu J, Horiuchi M, Pleasure D, Guo F (2013) Adenomatous Polyposis Coli Regulates Oligodendroglial Development. The Journal of Neuroscience 33:3113-3130.

Lee Y, Morrison BM, Li Y, Lengacher S, Farah MH, Hoffman PN, Liu Y, Tsingalia A, Jin L, Zhang P-W, Pellerin L, Magistretti PJ, Rothstein JD (2012) Oligodendroglia metabolically support axons and contribute to neurodegeneration. Nature advance online publication.

Li J, Ghiani CA, Kim JY, Liu A, Sandoval J, DeVellis J, Casaccia-Bonnefil P (2008) Inhibition of p53 transcriptional activity: a potential target for future development of therapeutic strategies for primary demyelination. J Neurosci 28:6118-6127.

Liao Y-C, Lo SH (2021) Tensins - emerging insights into their domain functions, biological roles and disease relevance. Journal of Cell Science 134:jcs254029.

Liao Y-C, Si L, deVere White RW, Lo SH (2007) The phosphotyrosine-independent interaction of DLC-1 and the SH2 domain of cten regulates focal adhesion localization and growth suppression activity of DLC-1. The Journal of cell biology 176:4349. 
bioRxiv preprint doi: https://doi.org/10.1101/2022.02.25.481980; this version posted February $27,2022$. The copyright holder for this preprint (which was not certified by peer review) is the author/funder, who has granted bioRxiv a license to display the preprint in perpetuity. It is made available under aCC-BY-NC-ND 4.0 International license.

Liu C, Sage Jonathan C, Miller Michael R, Verhaak Roel GW, Hippenmeyer S, Vogel H, Foreman O, Bronson Roderick T, Nishiyama A, Luo L, Zong H (2011) Mosaic Analysis with Double Markers Reveals Tumor Cell of Origin in Glioma. Cell 146:209-221.

Lu QR, Sun T, Zhu Z, Ma N, Garcia M, Stiles CD, Rowitch DH (2002) Common developmental requirement for Olig function indicates a motor neuron/oligodendrocyte connection. Cell 109:75-86.

Lu QR, Yuk D, Alberta JA, Zhu Z, Pawlitzky I, Chan J, McMahon AP, Stiles CD, Rowitch DH (2000) Sonic hedgehog--regulated oligodendrocyte lineage genes encoding bHLH proteins in the mammalian central nervous system. Neuron 25:317-329.

Luo F, Zhang Z, Luo Y (2021) Differential Role of p53 in Oligodendrocyte Survival in Response to Various Stresses: Experimental Autoimmune Encephalomyelitis, Cuprizone Intoxication or White Matter Stroke. International Journal of Molecular Sciences 22:12811.

Marie C, Clavairoly A, Frah M, Hmidan H, Yan J, Zhao C, Van Steenwinckel J, Daveau R, Zalc B, Hassan B, Thomas J-L, Gressens P, Ravassard P, Moszer I, Martin DM, Lu QR, Parras C (2018) Oligodendrocyte precursor survival and differentiation requires chromatin remodeling by Chd7 and Chd8. Proceedings of the National Academy of Sciences 115 (35).

Marques S et al. (2016) Oligodendrocyte heterogeneity in the mouse juvenile and adult central nervous system. Science 352:1326-1329.

Martuszewska D, Ljungberg B, Johansson M, Landberg G, Oslakovic C, Dahlbäck B, Hafizi S (2009) Tensin3 Is a Negative Regulator of Cell Migration and All Four Tensin Family Members Are Downregulated in Human Kidney Cancer. PLoS ONE 4:e4350.

McKenzie IA, Ohayon D, Li H, Paes de Faria J, Emery B, Tohyama K, Richardson WD (2014) Motor skill learning requires active central myelination. Science 346:318-322.

Meyer N, Richter N, Fan Z, Siemonsmeier G, Pivneva T, Jordan P, Steinhäuser C, Semtner M, Nolte C, Kettenmann H (2018) Oligodendrocytes in the Mouse Corpus Callosum Maintain Axonal Function by Delivery of Glucose. Cell Reports 22:2383-2394.

Mount CW, Monje M (2017) Wrapped to Adapt: Experience-Dependent Myelination. Neuron 95:743-756.

Nait-Oumesmar B, Decker L, Lachapelle F, Avellana-Adalid V, Bachelin C, Baron-Van Evercooren A (1999) Progenitor cells of the adult mouse subventricular zone proliferate, migrate and differentiate into oligodendrocytes after demyelination. Eur J Neurosci 11:4357-4366.

Nakatani H, Martin E, Hassani H, Clavairoly A, Maire CL, Viadieu A, Kerninon C, Delmasure A, Frah M, Weber M, Nakafuku M, Zalc B, Thomas JL, Guillemot F, Nait-Oumesmar B, Parras C (2013) Ascl1/Mash1 Promotes Brain Oligodendrogenesis during Myelination and Remyelination. J Neurosci 33:9752-9768.

Nawaz S, Sánchez P, Schmitt S, Snaidero N, Mitkovski M, Velte C, Brückner Bastian R, Alexopoulos I, Czopka T, Jung Sang Y, Rhee Jeong S, Janshoff A, Witke W, Schaap Iwan AT, Lyons David A, Simons M (2015) Actin Filament Turnover Drives Leading Edge Growth during Myelin Sheath Formation in the Central Nervous System. Developmental Cell 34:139-151.

Neumann B, Segel M, Chalut KJ, Franklin RJM (2019) Remyelination and ageing: Reversing the ravages of time. Multiple Sclerosis Journal 25:1835-1841.

Nishino T, Sasaki N, Chihara M, Nagasaki K-i, Torigoe D, Kon Y, Agui T (2012) Distinct Distribution of the Tensin Family in the Mouse Kidney and Small Intestine. Experimental Animals 61:525-532.

Nott $A$ et al. (2019) Brain cell type-specific enhancer-promoter interactome maps and disease-risk association. Science 366:1134-1139.

Pan S, Mayoral SR, Choi HS, Chan JR, Kheirbek MA (2020) Preservation of a remote fear memory requires new myelin formation. Nature Neuroscience 23:487-499.

Parras C, Marie C, Zhao C, Lu QR (2020) Chromatin remodelers in oligodendroglia. Glia 68: :1604- 1618.

Phan BN et al. (2020) A myelin-related transcriptomic profile is shared by Pitt-Hopkins syndrome models and human autism spectrum disorder. Nature Neuroscience 23:375-385.

Qi Y, Cai J, Wu Y, Wu R, Lee J, Fu H, Rao M, Sussel L, Rubenstein J, Qiu M (2001) Control of oligodendrocyte differentiation by the Nkx2.2 homeodomain transcription factor. Development 128:2723-2733.

Robinson JT, Thorvaldsdóttir H, Winckler W, Guttman M, Lander ES, Getz G, Mesirov JP (2011) Integrative genomics viewer. Nature Biotechnology 29:24-26. 
bioRxiv preprint doi: https://doi.org/10.1101/2022.02.25.481980; this version posted February $27,2022$. The copyright holder for this preprint (which was not certified by peer review) is the author/funder, who has granted bioRxiv a license to display the preprint in perpetuity. It is made available under aCC-BY-NC-ND 4.0 International license.

Steadman PE, Xia F, Ahmed M, Mocle AJ, Penning ARA, Geraghty AC, Steenland HW, Monje M, Josselyn SA, Frankland PW (2019) Disruption of Oligodendrogenesis Impairs Memory Consolidation in Adult Mice. Neuron.

Stolt CC, Rehberg S, Ader M, Lommes P, Riethmacher D, Schachner M, Bartsch U, Wegner M (2002) Terminal differentiation of myelin-forming oligodendrocytes depends on the transcription factor Sox10. Genes Dev 16:165-170.

Stuart T, Butler A, Hoffman P, Hafemeister C, Papalexi E, Mauck WM, Hao Y, Stoeckius M, Smibert P, Satija R (2019) Comprehensive Integration of Single-Cell Data. Cell 177:1888-1902.e1821.

Sun LO, Mulinyawe SB, Collins HY, Ibrahim A, Li Q, Simon DJ, Tessier-Lavigne M, Barres BA (2018) Spatiotemporal Control of CNS Myelination by Oligodendrocyte Programmed Cell Death through the TFEB-PUMA Axis. Cell 175:1811-1826.e1821.

Suzuki SO, Goldman JE (2003) Multiple cell populations in the early postnatal subventricular zone take distinct migratory pathways: a dynamic study of glial and neuronal progenitor migration. J Neurosci 23:4240-4250.

Thomason EJ, Escalante M, Osterhout DJ, Fuss B (2020) The oligodendrocyte growth cone and its actin cytoskeleton: A fundamental element for progenitor cell migration and CNS myelination. Glia 68:1329-1346.

Trapp BD, Nishiyama A, Cheng D, Macklin W (1997) Differentiation and Death of Premyelinating Oligodendrocytes in Developing Rodent Brain. Journal of Cell Biology 137:459-468.

Wang F, Ren S-Y, Chen J-F, Liu K, Li R-X, Li Z-F, Hu B, Niu J-Q, Xiao L, Chan JR, Mei F (2020) Myelin degeneration and diminished myelin renewal contribute to age-related deficits in memory. Nature Neuroscience 23:481-486.

Wheeler NA, Fuss B (2016) Extracellular cues influencing oligodendrocyte differentiation and (re)myelination. Experimental Neurology.

Wosik K, Antel J, Kuhlmann T, Brück W, Massie B, Nalbantoglu J (2003) Oligodendrocyte injury in multiple sclerosis: a role for p53. J Neurochem 85:635-644.

Xiao L, Ohayon D, McKenzie IA, Sinclair-Wilson A, Wright JL, Fudge AD, Emery B, Li H, Richardson WD (2016) Rapid production of new oligodendrocytes is required in the earliest stages of motor-skill learning. Nat Neurosci 19:1210-1217.

Xin W, Chan JR (2020) Myelin plasticity: sculpting circuits in learning and memory. Nature Reviews Neuroscience 21:682-694.

Yang SM, Michel K, Jokhi V, Nedivi E, Arlotta P (2020) Neuron class-specific responses govern adaptive myelin remodeling in the neocortex. Science 370:eabd2109.

Yu Y, Chen Y, Kim B, Wang H, Zhao C, He X, Liu L, Liu W, Wu Lai Man N, Mao M, Chan Jonah R, Wu J, Lu QR (2013) Olig2 Targets Chromatin Remodelers to Enhancers to Initiate Oligodendrocyte Differentiation. Cell 152:248-261.

Zaret KS, Mango SE (2016) Pioneer transcription factors, chromatin dynamics, and cell fate control. Curr Opin Genet Dev 37:76-81.

Zeisel A, Muñoz-Manchado AB, Codeluppi S, Lönnerberg P, La Manno G, Juréus A, Marques S, Munguba H, He L, Betsholtz C, Rolny C, Castelo-Branco G, Hjerling-Leffler J, Linnarsson S (2015) Cell types in the mouse cortex and hippocampus revealed by single-cell RNA-seq. Science 347:1138-1142.

Zhao C, Dong C, Frah M, Deng Y, Marie C, Zhang F, Xu L, Ma Z, Dong X, Lin Y, Koenig S, Nait-Oumesmar B, Martin DM, Wu LN, Xin M, Zhou W, Parras C, Lu QR (2018) Dual Requirement of CHD8 for Chromatin Landscape Establishment and Histone Methyltransferase Recruitment to Promote CNS Myelination and Repair. Developmental Cell,:753-768.e758.

Zhou Q, Anderson DJ (2002) The bHLH transcription factors OLIG2 and OLIG1 couple neuronal and glial subtype specification. Cell 109:61-73.

Zhou Q, Wang S, Anderson DJ (2000) Identification of a Novel Family of Oligodendrocyte Lineage-Specific Basic Helix-LoopHelix Transcription Factors. Neuron 25:331-343.

Zuchero JB, Fu M-m, Sloan Steven A, Ibrahim A, Olson A, Zaremba A, Dugas Jason C, Wienbar S, Caprariello Andrew V, Kantor C, Leonoudakus D, Lariosa-Willingham K, Kronenberg G, Gertz K, Soderling Scott H, Miller Robert H, Barres Ben A (2015) CNS Myelin Wrapping Is Driven by Actin Disassembly. Developmental Cell 34:152-167. 
Cioure 1 bioRxiv preprint doi: https://doi.org/10.1101/2022.02.25.481980; this version posted February 27, 2022. The copyright holder for this 1 preprint (which was not certified by peer review) is the author/funder, who has granted bioRxiv a license to display the preprint in perpetuity. It is made available under aCC-BY-NC-ND 4.0 International license.

A

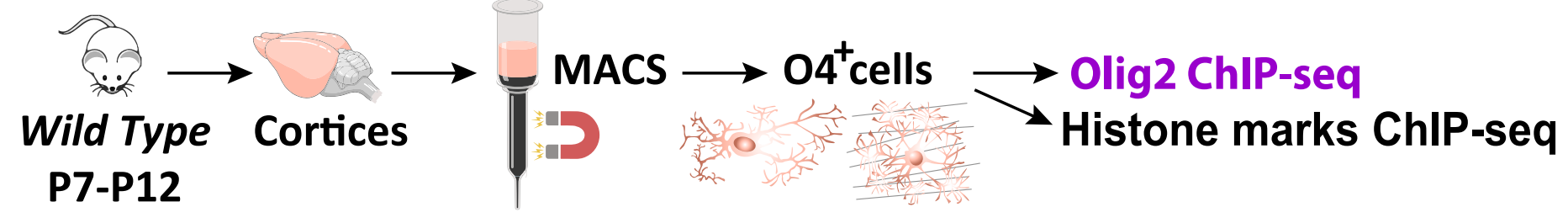

B

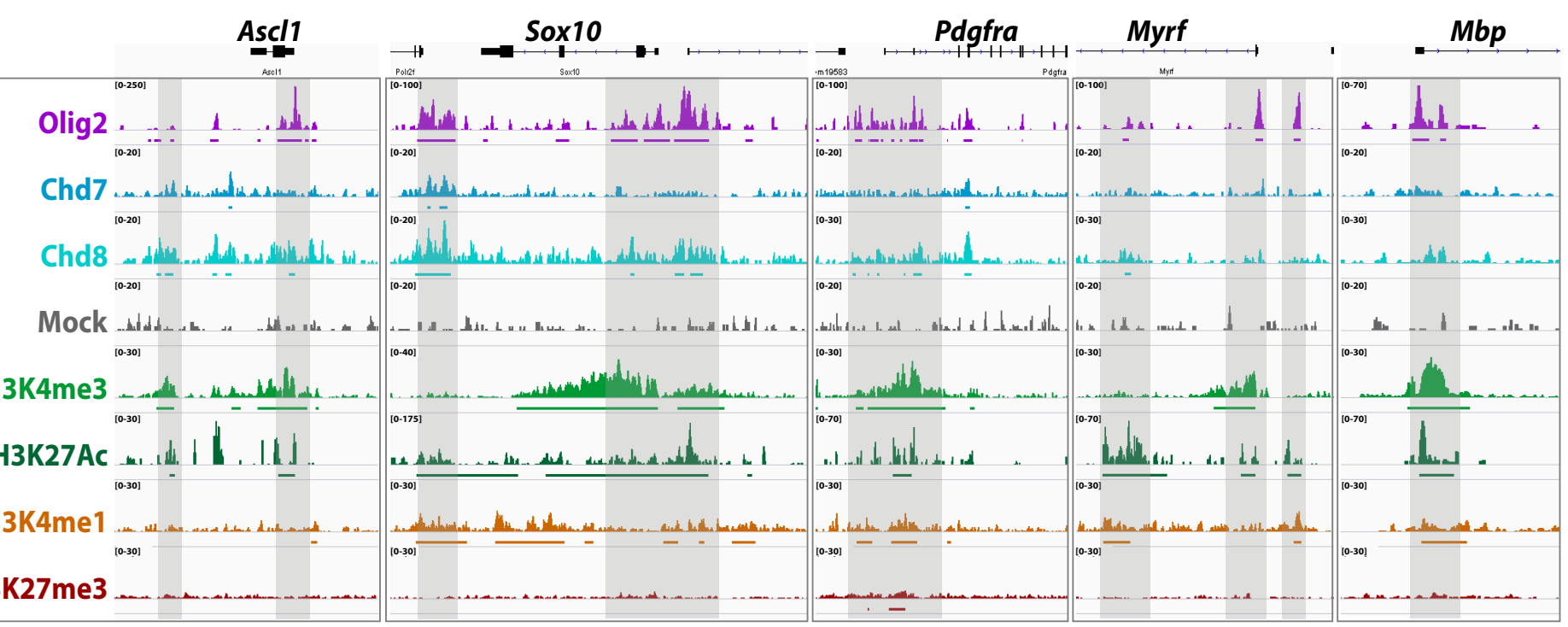

C

Binding sites

Chd7

5310
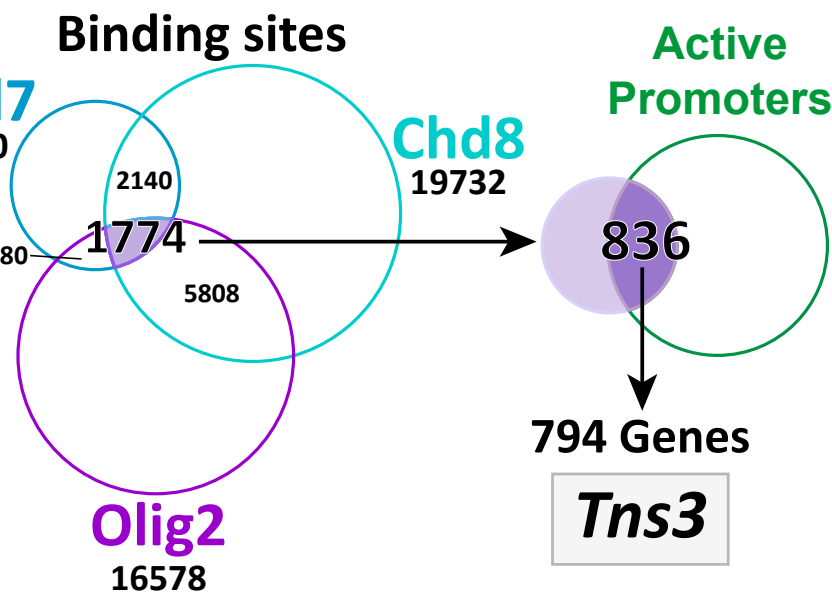

D

Mical3 3 Trca1

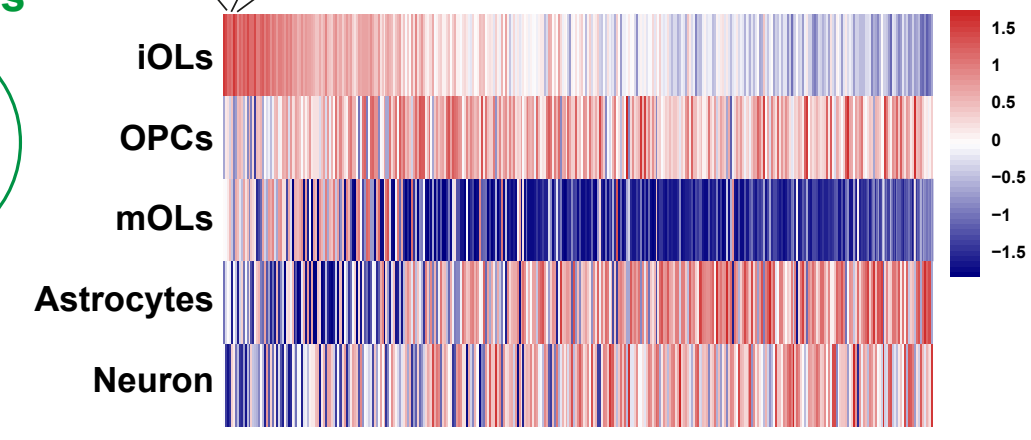

E

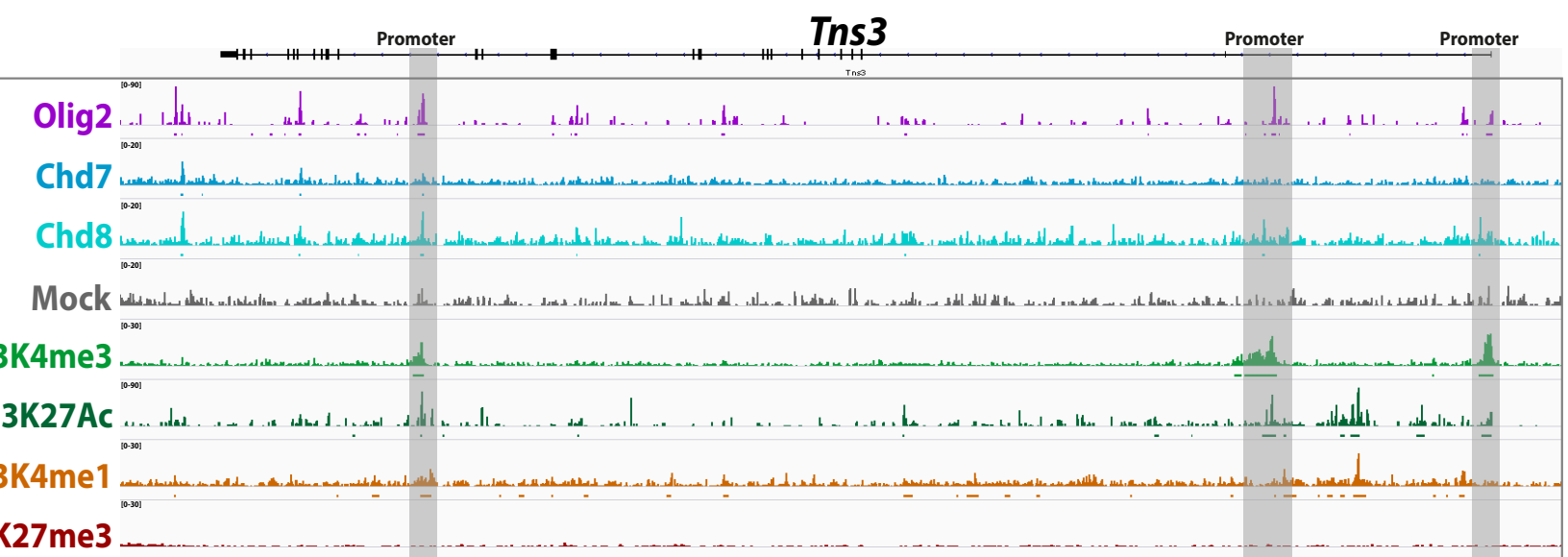
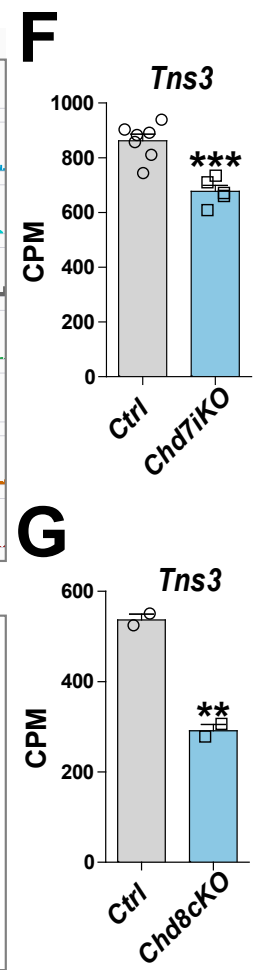
Figure 1. Tns3 is a target gene of Olig2 and Chd7/8 regulators of oligodendrocyte differentiation. (A) Scheme representing MACSorting of $\mathrm{O}^{+}$cells from wild type cortices followed by ChIP-seq for Olig2. (B) Schematic from IGV browser of Ascl1, Sox10, Pdgfra, Myrf and $\mathrm{Mbp}$ gene regions depicturing ChIP-seq data in $\mathrm{O}^{+}$cells (OPCs/OLs) for transcription factor Olig2, chromatin remodelers Chd7 and Chd8 and epigenetic marks (H3K4me3, H3K27ac, H3K4me1 and H3K27me3). Mock (control IgG) shows no peaks in the regions of interest. Lines present below peaks indicate statistical significance (peak calling). (C) Strategy used to identify Tns3 as a gene target of Olig2, Chd7, and Chd8, potentially involved in oligodendrogenesis. Left, Venn diagrams depicting the overlap of binding peaks between Chd7 (blue), Chd8 (cyan) and Olig2 (purple) in $\mathrm{O}^{+}$cells. Right, Venn diagram showing that 836 (47\%) of the 1774 common regions have marks of active promoters, corresponding to 794 genes, including Tns3. (D) Heatmap representing the expression of the 794 genes in iOLs compared to OPCs, mOLs, astrocytes and neurons. Tns3 is the third most specific. Data from Zhang et al., 2014 (E) Schematic from IGV browser of Tns3 gene region depicturing ChIPseq data in $\mathrm{O}^{+}$cells (OPCs/OLs) for transcription factor Olig2 and epigenetic marks (H3K4me3, $\mathrm{H} 3 \mathrm{~K} 27 \mathrm{ac}, \mathrm{H} 3 \mathrm{~K} 4 \mathrm{me} 1$ and H3K27me3), zooming in Tns3 alternative promoters. Mock (control $\lg G$ ) shows no peaks in the regions of interest. Lines present below peaks indicate statistical significance (peak calling). (F,G) Barplots showing Tns3 transcript count per million (CPM) in O4+ cells upon tamoxifen-induced Chd7 deletion (Chd7iKO, F) or Chd8 deletion (Chd8cKO, G) compared to control (Ctrl). Statistics were done using EdgeR suite. 
Figure 2
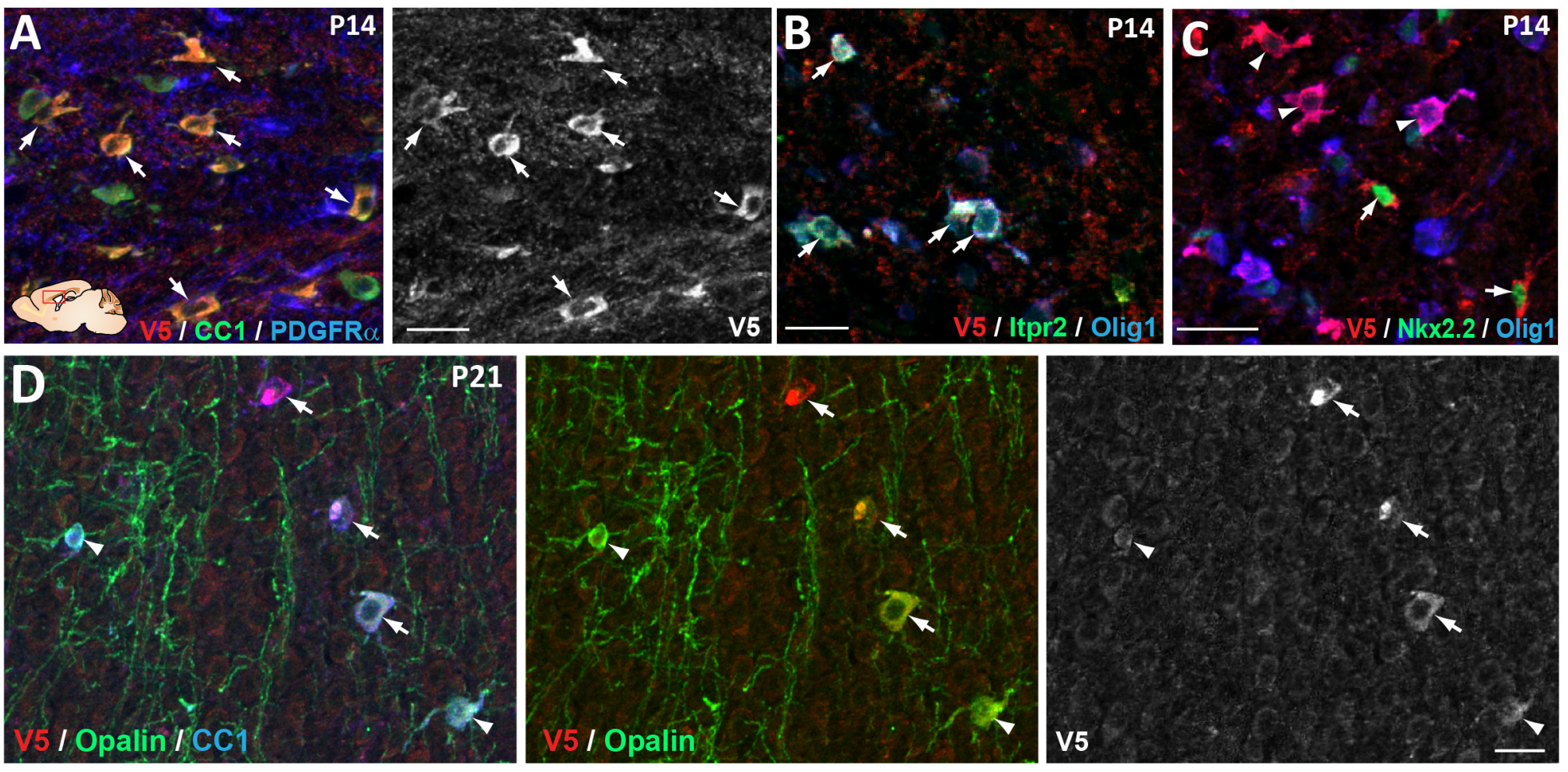

Y5 I Opalin / CC1
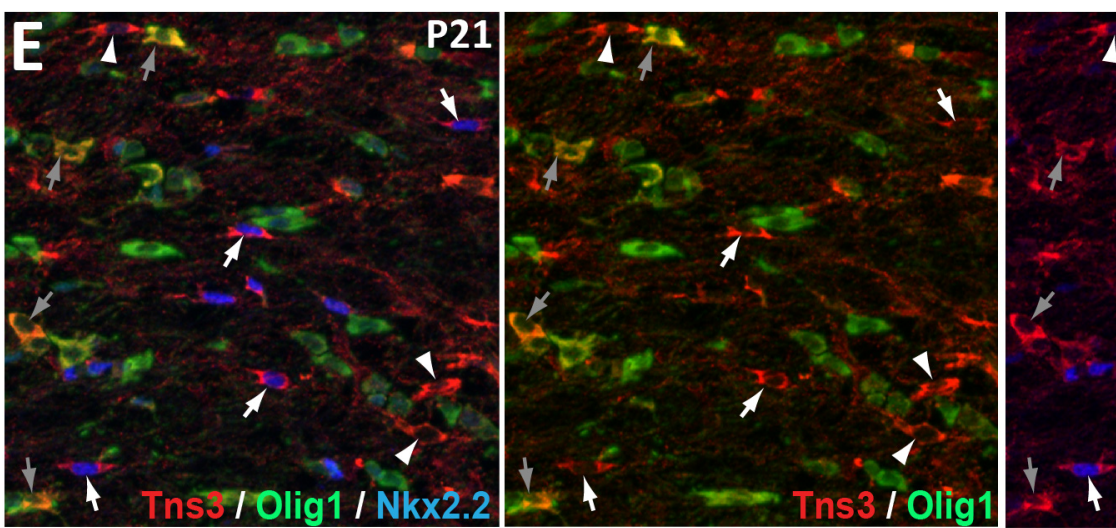

14

E

$F$

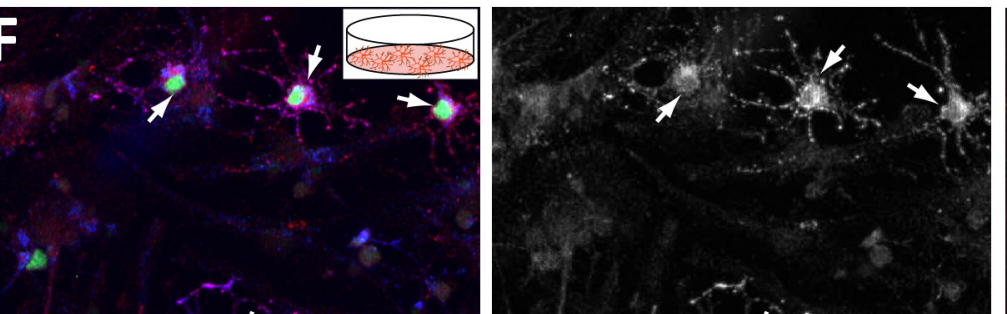

$$
5
$$

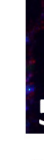

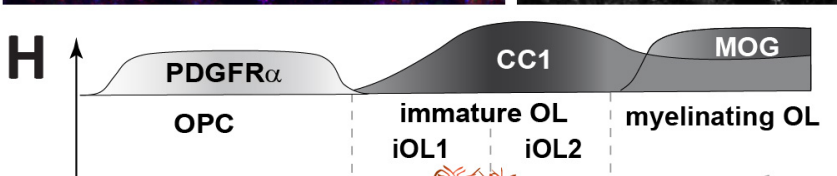
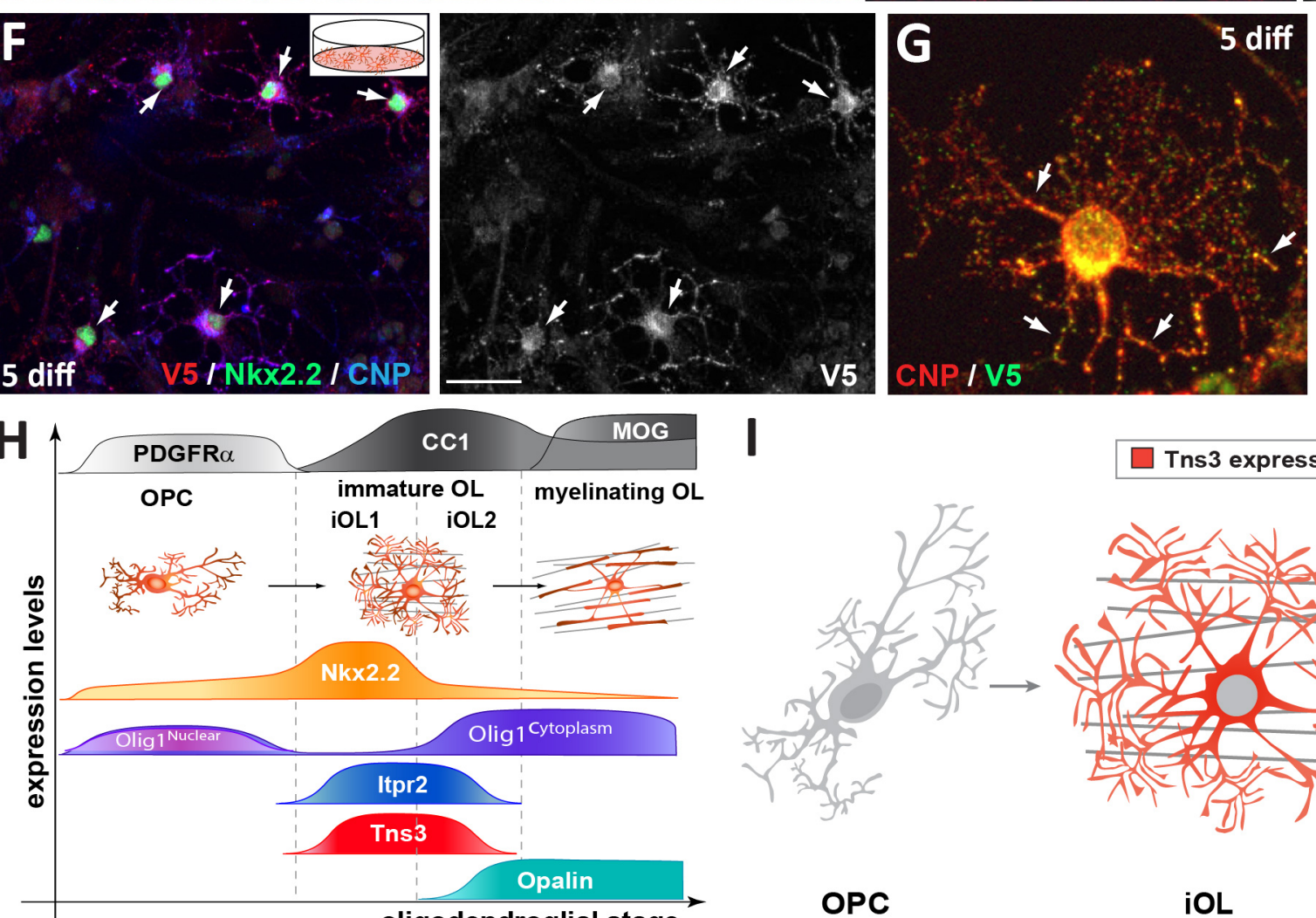

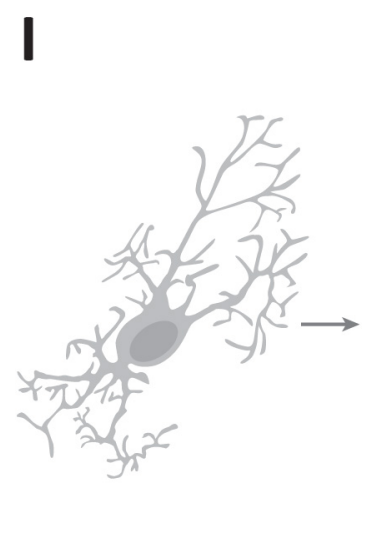

OPC

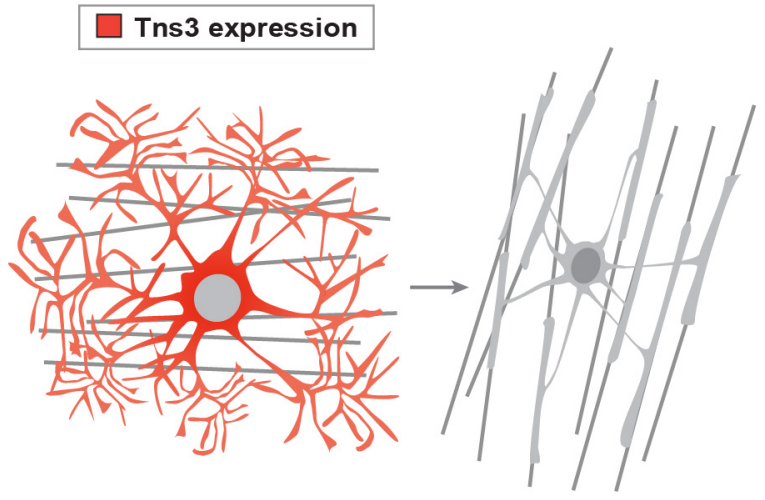

iOL

mOL 
Figure 2: Tns3 protein is detected at high levels in the cytoplasm and main cell processes of immature oligodendrocytes in the postnatal brain

Immunofluorescence in sagittal sections of postnatal brain at the level of the corpus callosum at P14 (A-C) and P21 (D-E) using V5 and Tns3 antibodies. Arrows indicate examples of labelled iOLs. (A) Tns3-V5 is detected at high levels in CC1+ OLs but not in PDGFR $\alpha+$ OPCs. (B) Tns3-V5 expression overlap well with Itpr2, with some of them being Olig $1^{\text {high-cytoplamic }}$ cells. (C) Tns3-V5 overlaps with high levels of $\mathrm{Nkx2.2}$ expression (arrows) and also in Nkx2.2\%/Olig1 ${ }^{\text {high-cytoplamic }}$ cells (arrowheads). (D) Tns3-V5 expression overlap with Opalin in iOLs (arrows, CC1 ${ }^{+}$cells with large cytoplasm) but is downregulated in Opalin ${ }^{+} \mathrm{mOLs}$ (arrowheads, $\mathrm{CC}^{+}$cells with small cytoplasm and myelin segments). (E) Tns3 (Sigma-Ct antibody) is detected at high levels in

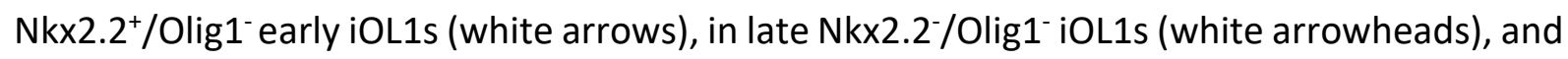
in Nkx2.2\%Olig1 high-cytoplamic iOL2s (grey arrows). (F) Tns3-V5 expression NSC cultures after 5 days in differentiation. Note the Tns3 expression in Nkx2.2 $/ \mathrm{CNP}^{+}$OLs (arrows). (G) Subcellular localization of Tns3 expression in $\mathrm{CNP}^{+}$OLs present in the cytoplasm and in dots distributed along the cell processes, overlapping with CNP signal (arrows). (H) Schematic representation of Tns3 expression together with key markers of different oligodendroglial stages summarizing data shown in A- E panels. (I) Schematic representation of Tns3 expression and subcellular localization in oligodendroglia. Scale bars: A-F, $20 \mu \mathrm{m} ; \mathrm{G}, 10 \mu \mathrm{m}$. 
Figure 3: Oligodendrogenesis is normal in Tns3 constitutive mutant mice, which still express Tns3 full-length transcripts in the brain. $\left(A, A^{\prime}\right)$ Immunofluorescence for PDGFR $\alpha^{+}$OPCs and $\mathrm{CC}^{+}$OLs showing similar cell numbers in the CC of $\operatorname{Tns} 3^{\beta g e o /+}(\mathbf{A})$ and $\operatorname{Tns} 3^{\beta g e o / \beta g e o}\left(\mathbf{A}^{\prime}\right)$ brains. (B) Histograms representing density of PDGFR $\alpha^{+} \mathrm{OPCs}$ and $\mathrm{CC} 1^{+} \mathrm{OLs}$ in the $\mathrm{CC}$ and the fimbria. (C, $\left.\mathbf{C}^{\prime}\right)$ Immunofluorescence for Olig2, CC1 and Olig1 allowing to identify three OL stages: CC1 $1^{\text {high }} / O$ lig1 $1^{-}$cells (named iOL1), CC1 $1^{\text {high }} / O$ Olig $1^{\text {high-cytoplasmic }}$ cells (iOL2) and CC1 ${ }^{\text {low }} / O$ lig $1^{\text {cytoplasmic }}$ (mOLs), showing similar numbers of each OL stage in the CC of Tns $3^{\beta g e o /+}$ (C) and Tns $3^{\beta g e o / \beta g e o}$ (C') brains. (D) Histograms representing the quantification of the density of iOL1s, iOL2s, and mOLs, in the CC and the fimbria. (E) Genotyping by TIDE analysis of Tns $3^{4 d e l}$ and Tns $3^{14 d e l}$ heterozygous mice showing wild type Tns3 allele and the 4 nucleotides deletion in Tns $3^{4 d e l}$ allele and the 14 nucleotides deletion in $T n s 3^{14 d e l}$ allele. (F) Pie charts representing the genotypes of E14.5 embryos obtained from Tns $3^{4 d e l}$ heterozygous inter-crosses. Note the sublethal phenotype indicated by the reduced number of $T n s 3^{4 d e / 4 d e l}$ embryos compared to Mendelian ratios. (G) Tns $3^{4 d e l}$ mice presenting growth defects in homozygous pups (arrow) at P14 compared to their heterozygous littermates. $(\mathbf{H}, \mathbf{I})$ Immunofluorescence showing that Tns3 is still detected in CC1+ OLs of Tns $3^{\text {4de/4del }}$ P21 mice, with four different Tns3 recognizing antibodies: (H) Sassan Hafizi lab and Santa Cruz, (I) Millipore and Sigma. (J) Histogram representing $\mathrm{qPCR}$ on cDNA from P21 brains showing no differences in the amplification of Tns3 Exon13-15, Exon 17 and Exon31 between Tns3 $3^{4 d e l / 4 d e l}$, Tns $3^{4 d e l /+}$ or WT mice, indicating the presence of a long Tns3 transcript containing these exons in Tns $3^{4 d e l / 4 d e l}$ brains. CC, corpus callosum. Scale bar, $20 \mu \mathrm{m}$. 
A
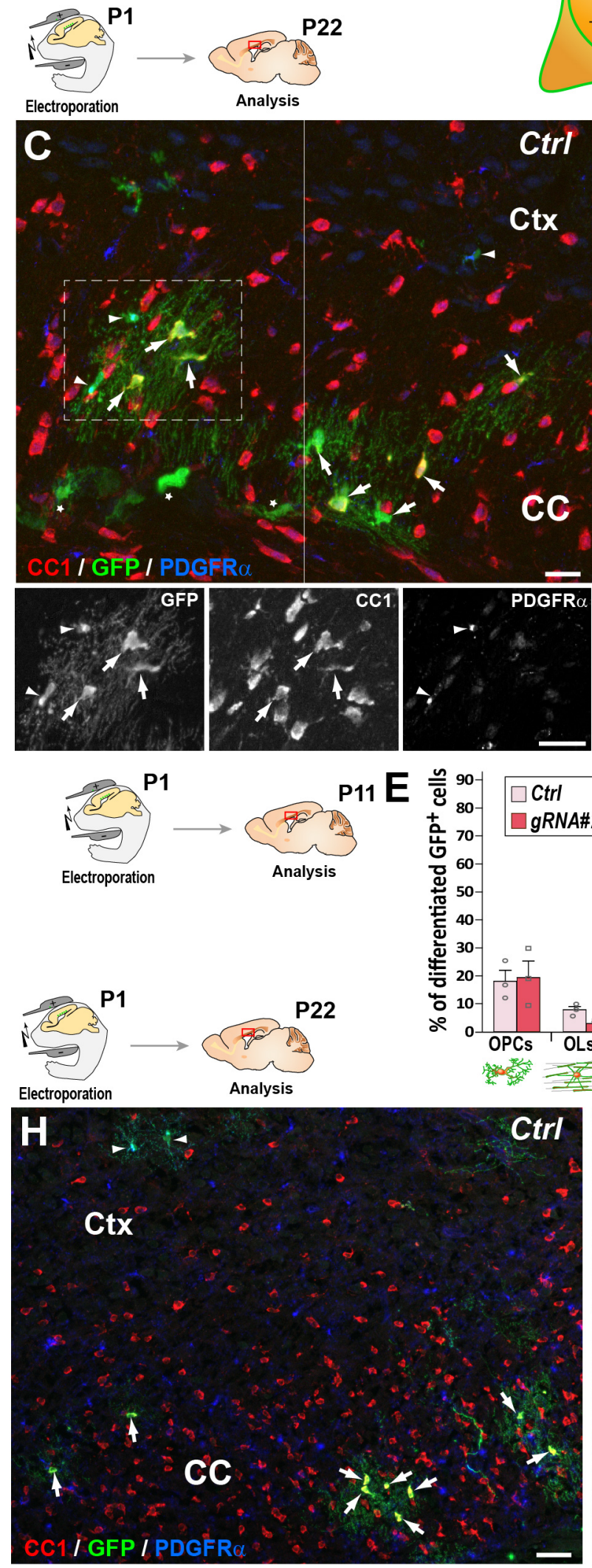

B

Integrative CRISPR/Cas9 vector To/2 pU6 $p C A G$

$2 \mathrm{~A} \quad \mathrm{Tol} 2$ GFP, P22

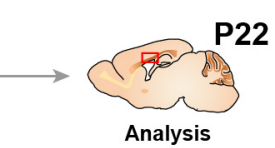

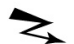

Tol2-gRNA-Cas9-GFP

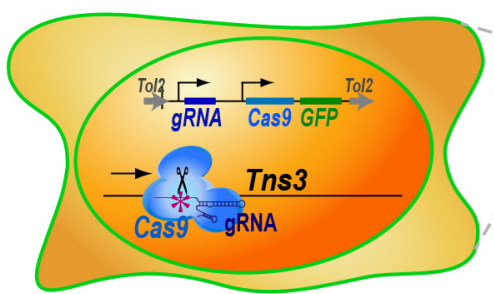

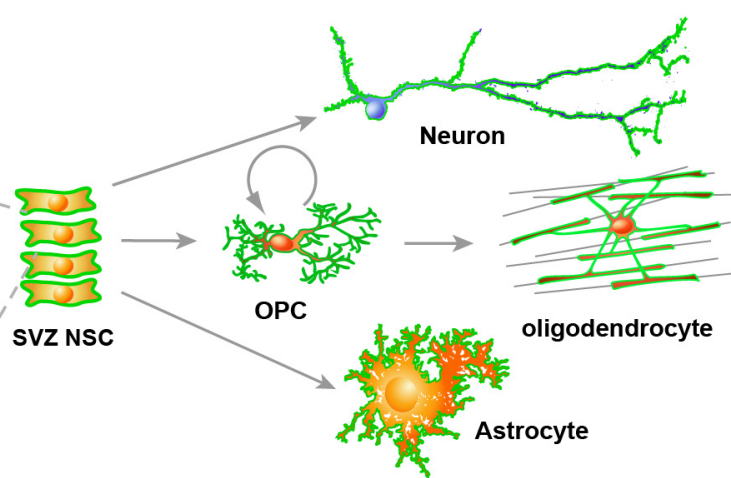

Mstrocyte
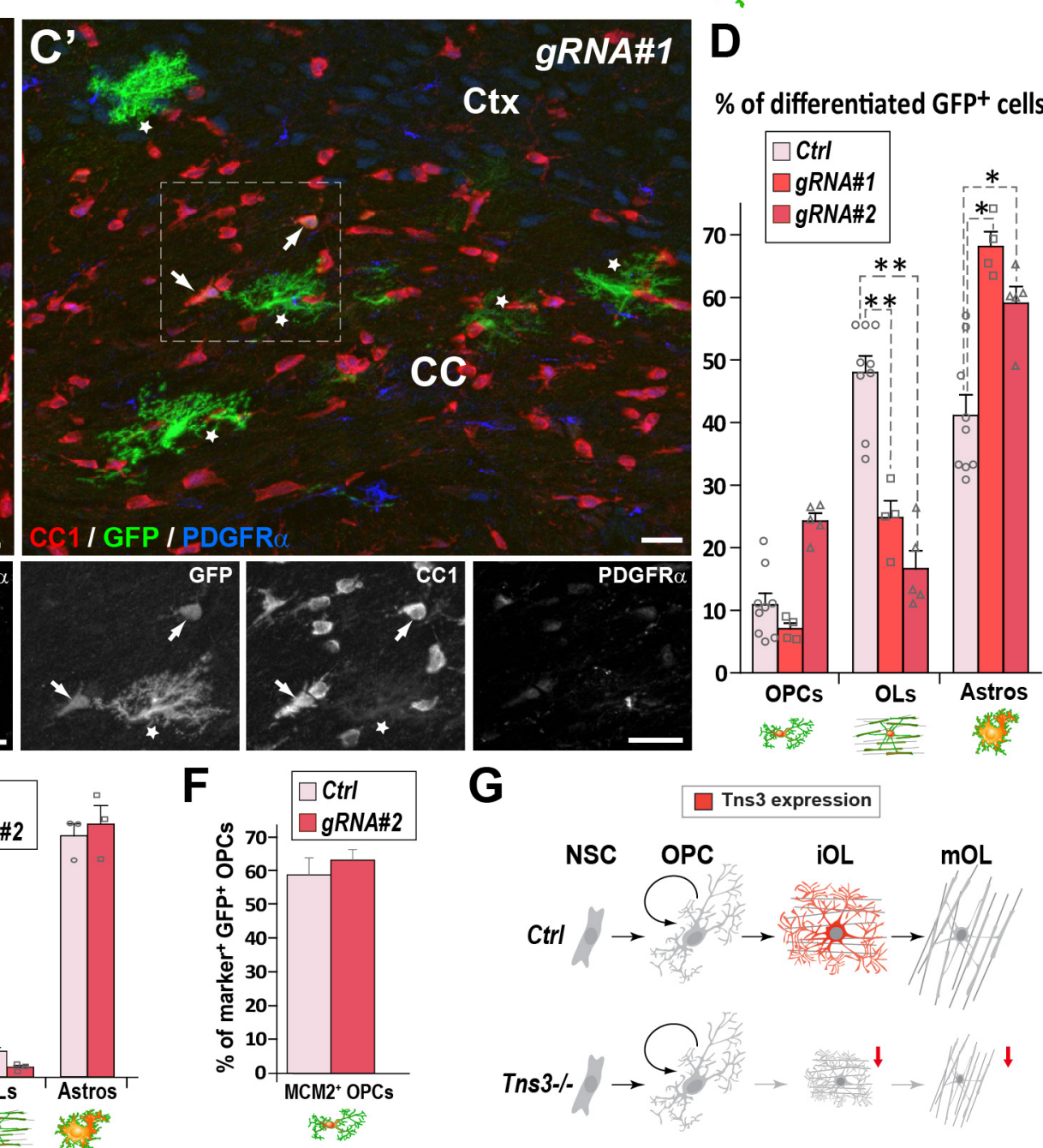

$\mathbf{G}$

$\square$ Tns3 expression

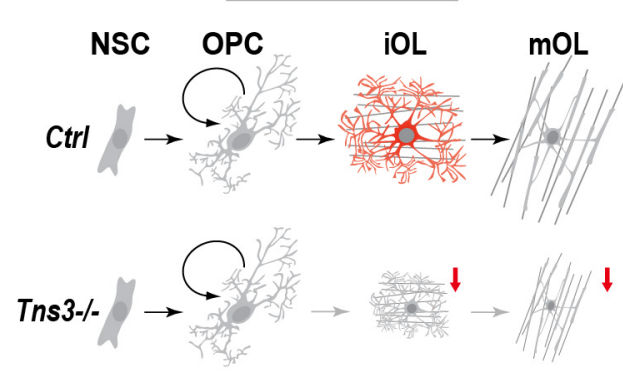

\section{5'-3' gRNAS I}

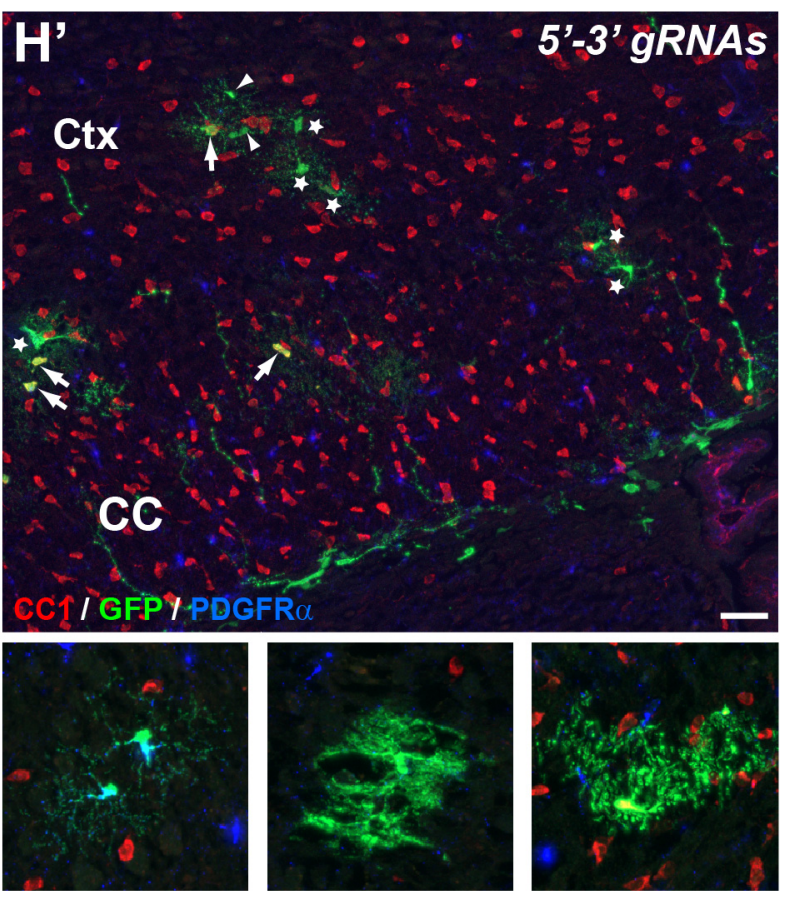

$\%$ of differentiated GFP+ cells

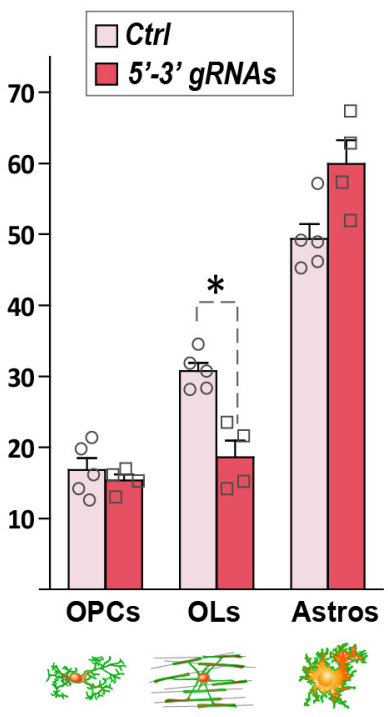


Figure 4: CRISPR-mediated Tns3 mutation in NSCs reduces oligodendrocyte differentiation in the postnatal brain. (A) Schematic of the CRISPR/Cas9 expression vector allowing Tol2-DNA integration driving Cas9 and GFP expression (from polycistronic 2A-mediated cleaved) from CAG promoter and sgRNA expression from U6 promoter. (B) Schematic of the dorsal SVZ electroporation of CRISPR-plasmid at postnatal day 1 (P1) and immunofluorescence analysis carried out in sagittal brain sections at P22. (C) Immunofluorescence of representative P22 sagittal sections of the dorsal telencephalon showing GFP+ cells being either PDGFR $\alpha^{+}$OPCs (arrowheads), CC1 $1^{\text {high }}$ OLs (arrows) or $\mathrm{CC} 1^{\text {low }}$ astrocytes (asterisks) progeny of P1 NSCS electroporated either with Ctrl plasmid (C) or Tns3-gRNA\#1 plasmid (C'). (D) Histogram showing the percentage of $\mathrm{GFP}^{+}$glial cell types found in Ctrl, gRNA\#1 or gRNA\#2 electroporated brains being PDGFR $\alpha^{+}-\mathrm{OPCs}, \mathrm{CC} 1^{\text {high }}$-OLs and $\mathrm{CC} 1^{\text {low }}$-astrocytes. Note the 2fold reduction of $\mathrm{CC}^{+}$OLs in Tns3-gRNA transfected brains, as illustrated in $\mathrm{D}^{\prime}$ compared to D. (F) Histograms representing the percentage of $\mathrm{GFP}^{+}$differentiated cells at P11. Note the lack of changes in OPCs, and the incipient reduction in OLs. (F) Histograms quantifying the proportion of proliferative ( $\mathrm{MCM}^{+}$cells) $\mathrm{GFP}^{+}$OPCs in electroporated P11 mice brain. (G) Schematic of Tns3 expression in mice (Upper) and of the effects of Tns3 CRISPR-mediated deletion (Lower). ( $\left.\mathbf{H}, \mathbf{H}^{\prime}\right)$ Representative P22 sagittal sections of the dorsal telencephalon showing $\mathrm{GFP}^{+}$cells being either PDGFR $\alpha^{+}$OPCs (arrowheads), CC1 $1^{\text {high }}$ OLs (arrows) or $\mathrm{CC} 1^{\text {low }}$ astrocytes (asterisks) progeny of P1 NSCs electroporated either with Ctrl plasmid (H) or Tns3$5^{\prime}-3^{\prime}$ targeting plasmid (labeled as 5'-3' gRNAs) ( $\left.\mathbf{H}^{\prime}\right)$. (I) Histograms showing the percentage of $\mathrm{GFP}^{+}$glial cell types found in Ctrl or $3^{\prime}-5^{\prime}$ gRNA electroporated brains being PDGFR $\alpha^{+}-\mathrm{OPCS}$, $\mathrm{CC} 1^{\text {high }}$ OLs and CC1 $1^{\text {low }}$ astrocytes in the corpus callosum (CC) and cortex (Ctx). Note the 2-fold reduction of $\mathrm{CC}^{+}$OLs in Tns3-5'-3' gRNA transfected brains. Scale bar, $20 \mu \mathrm{m}$. 
Figure 5: OPC-specific Tns3 deletion reduces the number of differentiating oligodendrocytes in the postnatal brain. (A) Scheme of tamoxifen administration to Tns3-iKO and control (Cre+; Tns $^{+/+}$) mice, Cre-mediated genetic changes, and timing of experimental analysis. (B-B', C-C') Immunofluorescence in P21 sagittal brain sections for CC1, GFP and PDGFR $\alpha$ illustrating similar density of OPCs and 2-fold reduction in OL density in Tns3-iKO $\left(\mathbf{B}^{\prime}, \mathbf{C}^{\prime}\right)$ compared to control (B,C) in the fimbria (B) and the cortex (C). (D) Histograms showing OPC and OL density in P21 Tns3-iKO and control (Ctrl) mice, in the corpus callosum, fimbria, cortex, and striatum. Note the systematic OLs decrease of $40-50 \%$ in each region. (E- $\left.\mathbf{E}_{1}{ }^{\prime}\right)$ Immunofluorescence in P21 sagittal brain sections for Olig1, GFP and CC1 to distinguish three stages of oligodendrogenesis: OPCs (Olig1 $\left.1^{+} / \mathrm{CC} 1^{-}\right)$, iOL1s (CC1 $\left.{ }^{+} / \mathrm{Olig} 1^{-}\right)$and $\mathrm{iOL} 2 \mathrm{~s} / \mathrm{mOLs}\left(\mathrm{CC} 1^{+} / \mathrm{Olig} 1^{+}\right)$in Ctrl (E) or Tns3-iKO mice (E'). $E_{1}$ and $E_{1}^{\prime}$ are higher magnification of the squared area in $E$ and $E^{\prime}$. (F) Histograms showing the OPCs, the iOL1s and the iOL2s/mOLs density in P21 Tns3-iKO and control mice, in the corpus callosum, fimbria, cortex, and striatum. Note the decrease of iOL1s and iOL2s over $40 \%$ in each area quantified (except for iOL1 density in the corpus callosum). (G) Schematic representing defects in oligodendrogenesis found in Tns3-iKO compared to control. CC, corpus callosum; Ctx, cortex. Scale bar, $20 \mu \mathrm{m}$. 
A

Tns3-iKO: Pdgfra-CreER'; Tns3 $3^{n \text {; }}$; Rosa26R

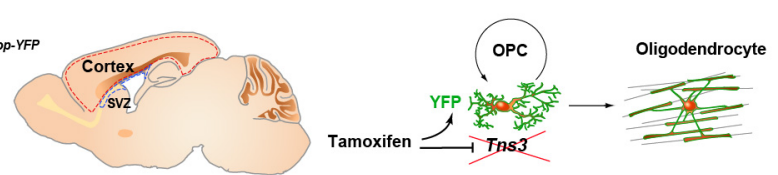

\section{B} Tamoxifen

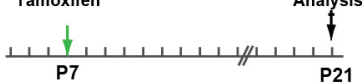

Tamoxifen

\section{Tns3-iKO}

Cortex

\section{Ctrl}

\section{$B^{\prime}$}

1
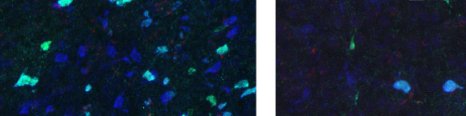

$\mathrm{B}^{2}$

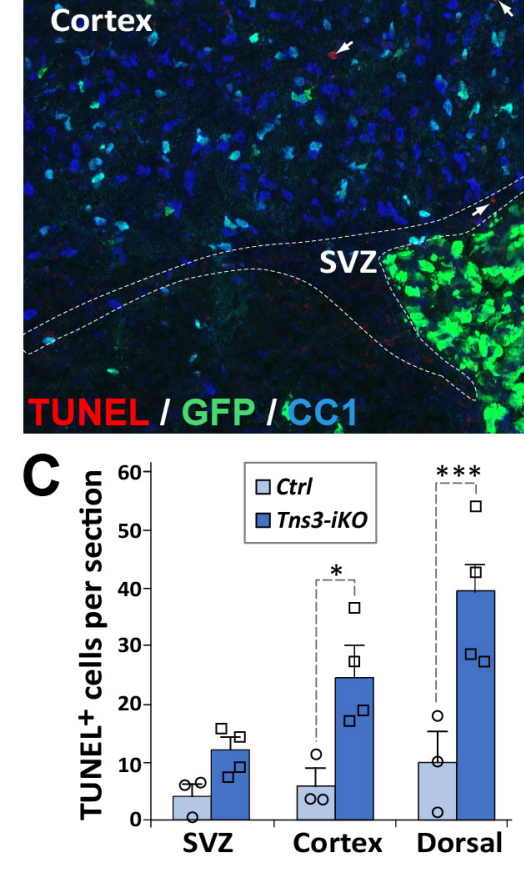

Cortex

\section{D}

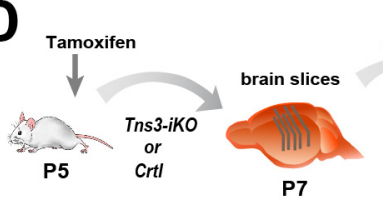

$E$

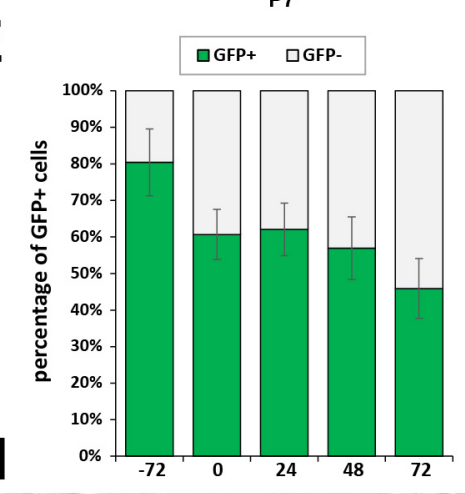

\section{c.}

cortex
dissection

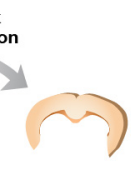
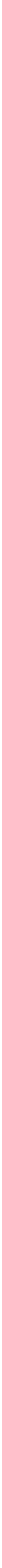

F

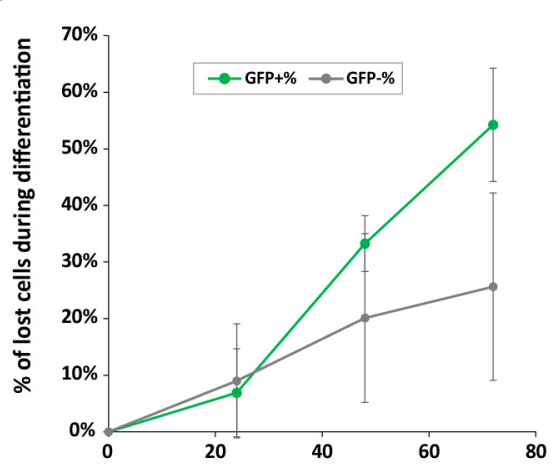

G

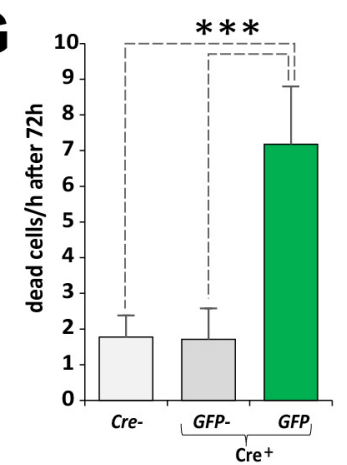

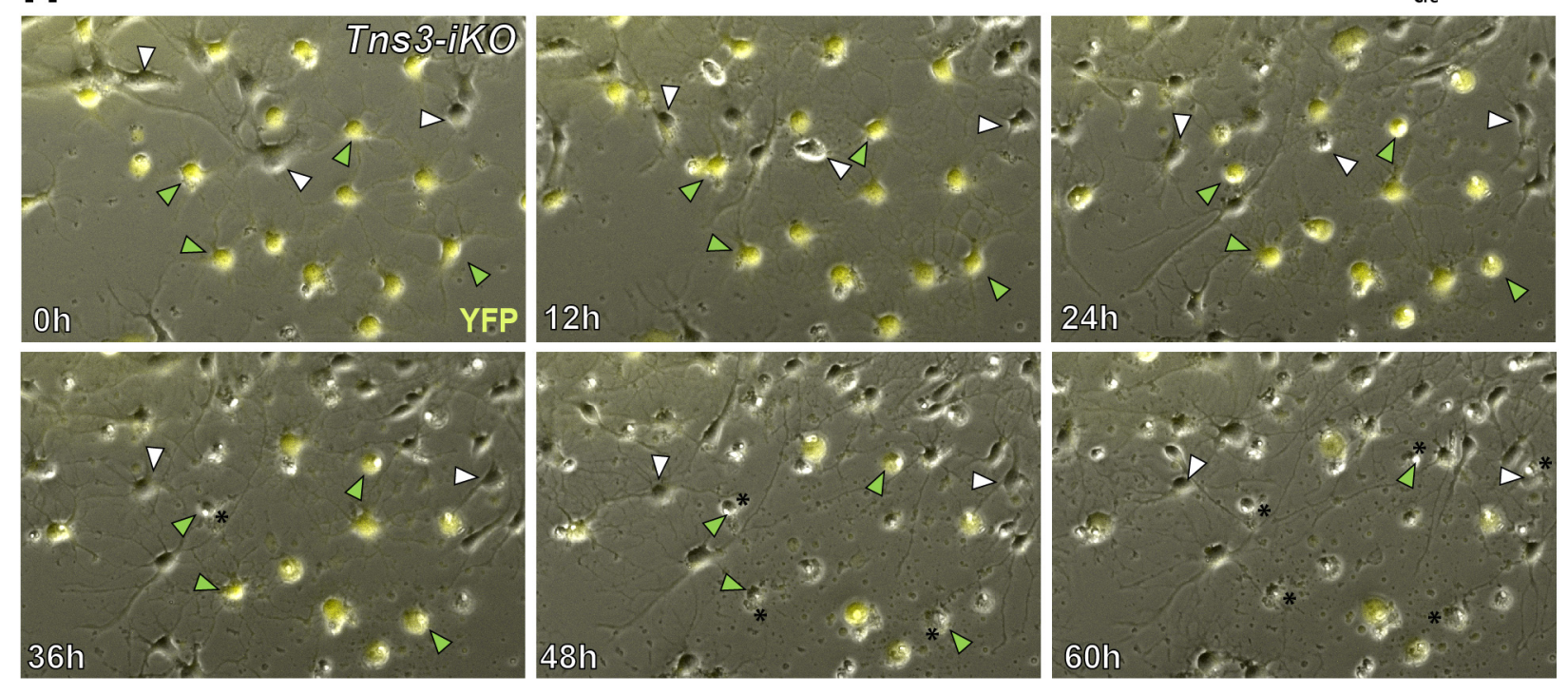

Figure 6 preprint (which was not certified by peer review) is the author/funder, who has granted bioRxiv a license to display th
perpetuity. It is made available under aCC-BY-NC-ND 4.0 International license.

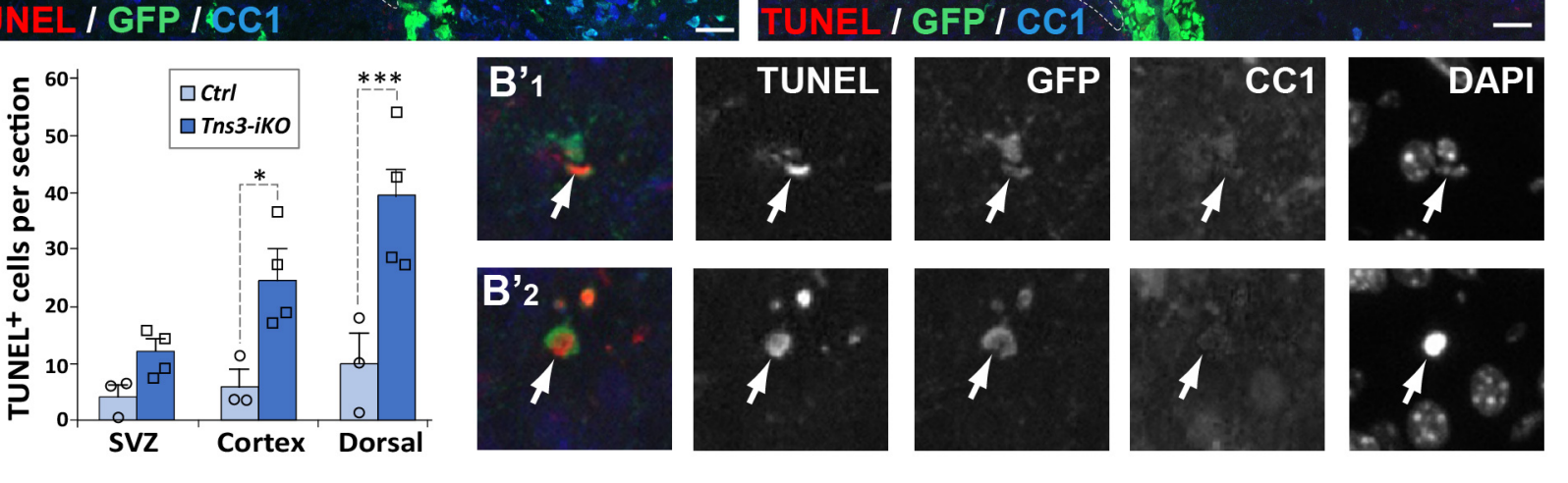


Figure 6 Increased cell death of Tns3-iKO oligodendroglia. (A) Scheme of tamoxifen administration to Tns3-iKO and control $\left(\mathrm{Cre}^{+} ; \mathrm{Tns}^{+/+}\right)$mice, Cre-mediated genetic changes, and timing of experimental analysis. (B-B') Immunodetection of death cells by TUNEL, together with recombined cells (GFP) and OLs (CC1+ cells) showing increase number of TUNEL cells in the cortex and corpus callosum of Tns3-iKO mice (B') compared to control mice (B), including some $\mathrm{GFP}^{+} / \mathrm{CC}^{-}$OPCs (insets $\mathrm{B}^{\prime}{ }_{1}$ and $\mathrm{B}^{\prime}{ }_{2}$ ). (C) Histograms quantifying the number of TUNEL ${ }^{+}$cells in the SVZ, cortex and both together (dorsal) per section. (D) Scheme of the video microscopy protocol in MACSorted OPCs purified from Tns3-iKO and control P7 mice. Cells were imaged every 10 minutes during $72 \mathrm{~h}$ in differentiation medium. (E) Histograms showing the reduction of the GFP+ cells proportion from the plating $(-72 h)$ to the end of the experiment (72h after differentiation onset). (F) Time curve quantifying the loss of GFP+ OLs compared to GFP- OLs during the $72 \mathrm{~h}$ of differentiation. (G) Histograms representing the quantification of cells lost per hour during the $72 \mathrm{~h}$ differentiation period, showing a 5-fold increase in loss of $\mathrm{GFP}^{+}$Tns3-iKO cells compared to GFP- cells (non-recombined cells from Tns3-iKO mice, internal negative control) or cells coming from $\mathrm{Cre}^{-}$littermates ( $\mathrm{Cre}^{-}$, external negative control). (H) Time lapse frames showing cells every 12 hours illustrating both GFP' (green arrowheads, recombined Tns3-iKO cells) and GFP- (white arrowheads, non-recombined Tns3-iKO cells) that die over the time of video microscopy. Note the larger number of GFP+ OLs (cells with multibranched OL morphology) dying compared to GFP- OLs. SVZ, subventricular zone. Scale bar, $20 \mu \mathrm{m}$. 


\section{Figure 7}

A Tns3-iKO: Pdgfra-CreERT'; Tns $3^{f / i t} ;$ Rosa26R
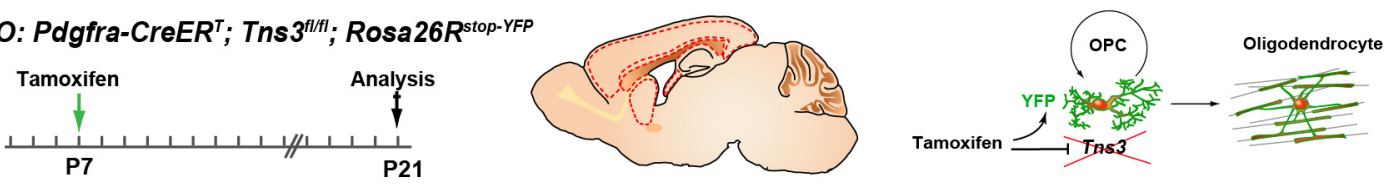

B :

\section{Tns3-1KO B'}

Ctrl

$\cdot x^{3}$

त)

Ctx

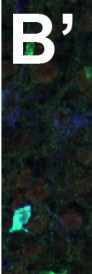

Ctx
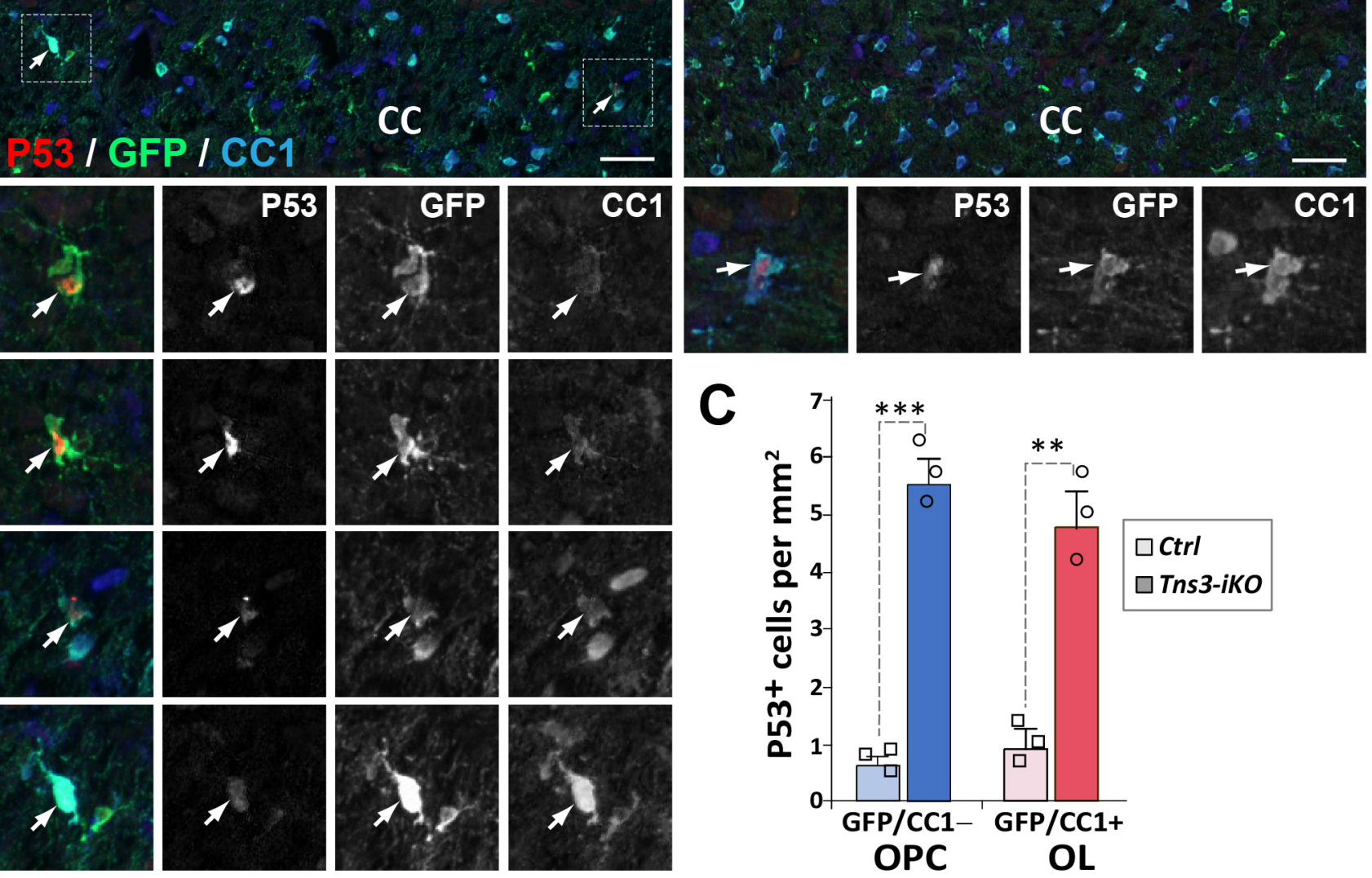

D
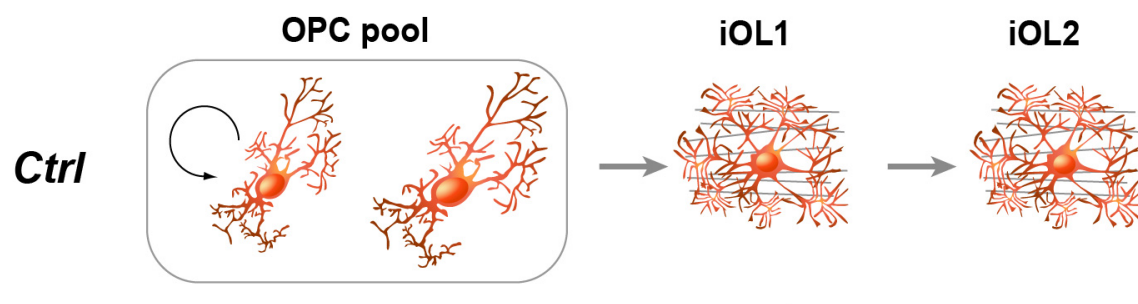

mOL

P53 $\uparrow$

Tns3-iKO
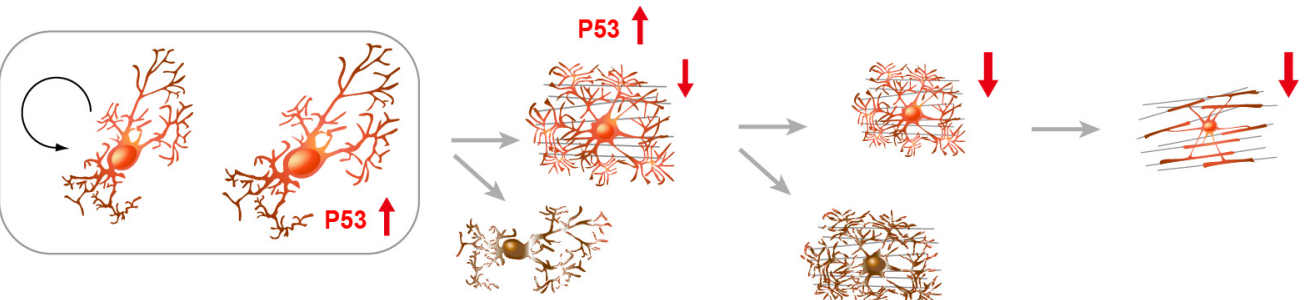
Figure 7. Mechanisms involved in Tns3-iKO oligodendroglial defects. (A) Scheme of tamoxifen administration to Tns3-iKO and control $\left(\mathrm{Cre}^{+} ; \mathrm{Tns}^{+/+}\right)$mice, Cre-mediated genetic changes, and timing of experimental analysis. (B-B') Immunodetection of p53, together with GFP to label recombined cells and CC1 to label OLs showing a strong increase number of $\mathrm{p} 53^{+} / \mathrm{GFP}^{+} / \mathrm{CC1}^{-}$OPCs and $\mathrm{p} 53^{+} / \mathrm{GFP}^{+} / \mathrm{CC1}^{+}$OLs in the cortex and corpus callosum of Tns3-iKO mice ( $\left.\mathbf{B}^{\prime}\right)$ compared to control mice (B). Dotted squares highlight some cases of p53+ cells, shown at higher magnification below. (C) Histograms quantifying the number of $\mathrm{p} 53^{+}$cells per area $\left(\mathrm{mm}^{2}\right)$ in the dorsal telencephalon. CC, corpus callosum; Ctx, cortex. Scale bar, $20 \mu \mathrm{m}$. (D) Schematics representing Tns3 function in oligodendrogenesis. CC, corpus callosum; Ctx, cortex. Scale bar, $20 \mu \mathrm{m}$. 
Figure SipRxiv preprint doi: https://doi.org/10.1101/2022.02.25.481980; this version posted February 27, 2022. The copyright holder for this foreprint (which was not certified by peer review) is the author/funder, who has granted bioRxiv a license to display the preprint in
perpetuity. It is made available under aCC-BY-NC-ND 4.0 International- license--

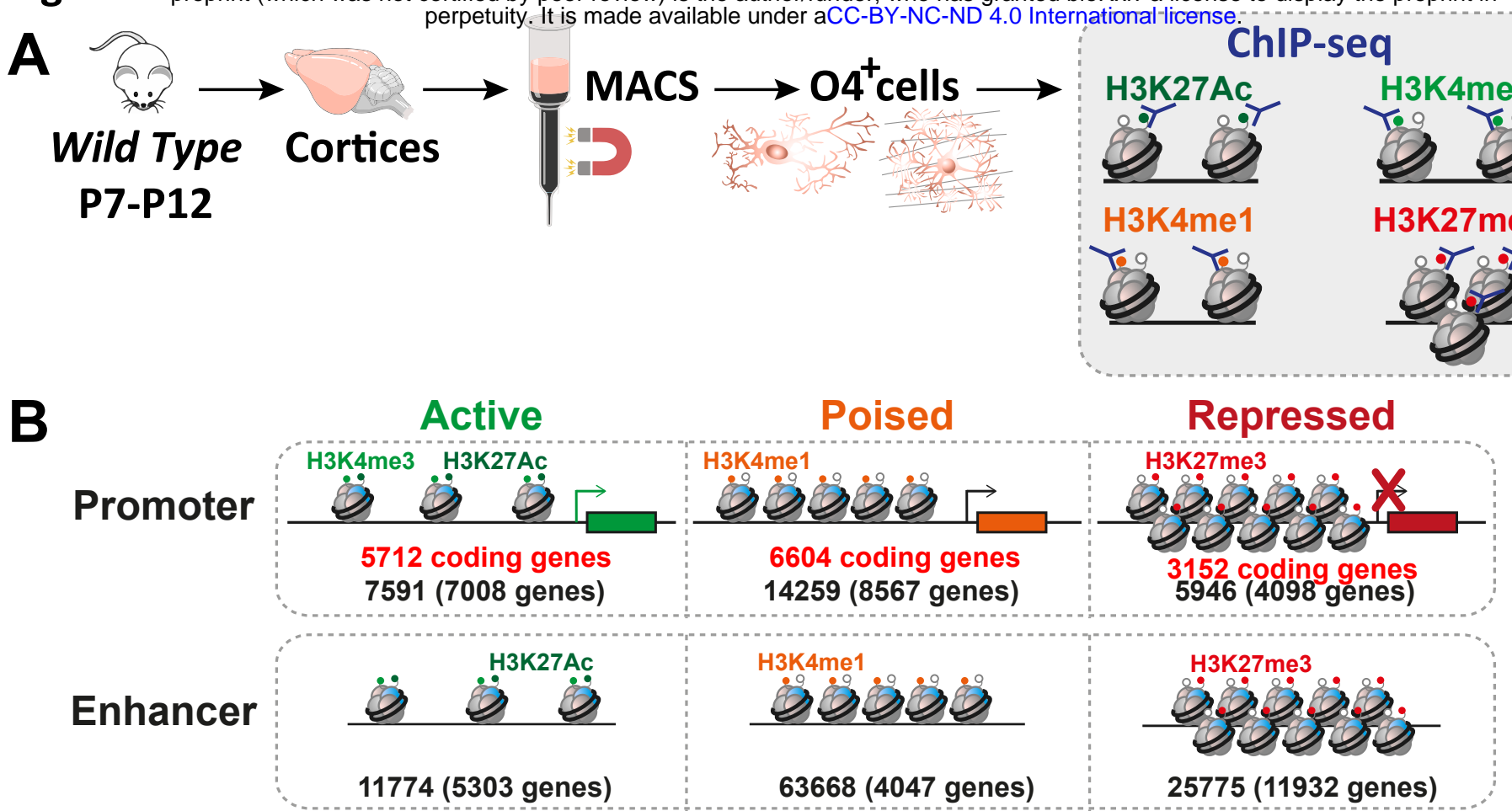

C

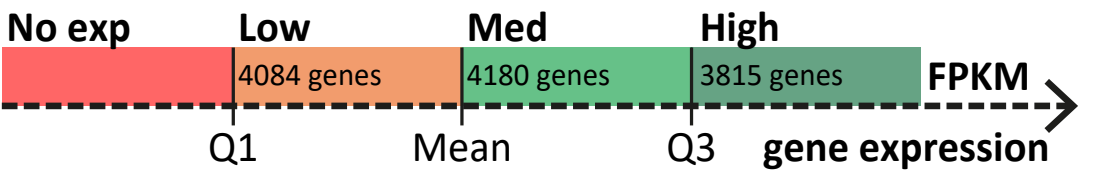

Gene expression: $\square$ Med/High Low No

D

Olig2 Replicate 2

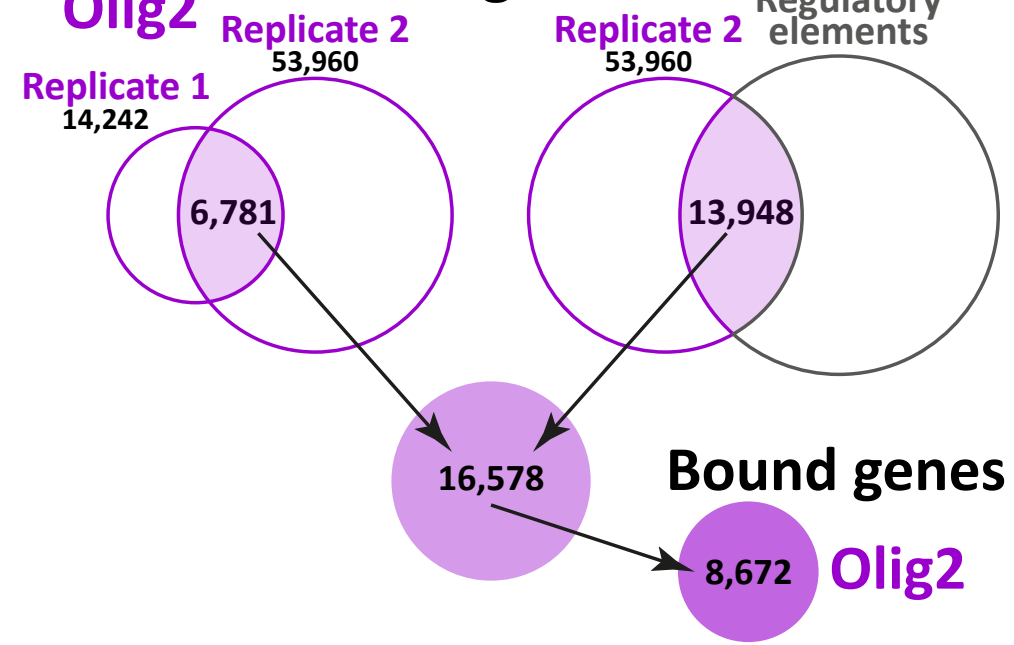

G Chd7 and/or Chd8

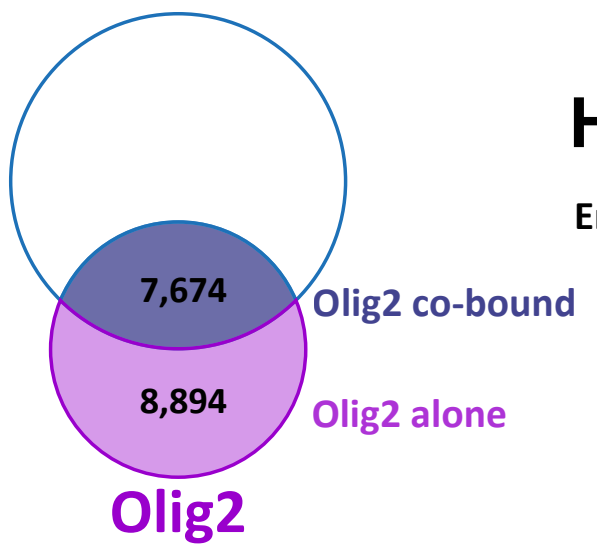

\section{H Olig2 co-bound}
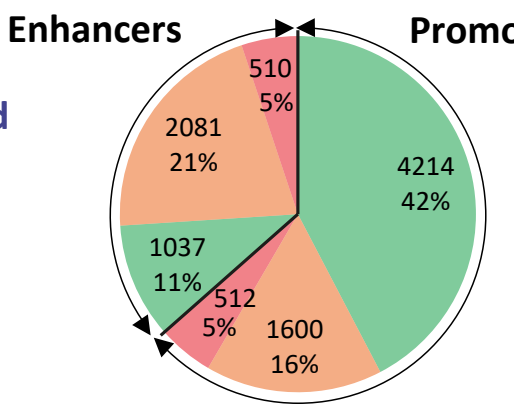

Active

Poised

Repressed

\section{Olig2}

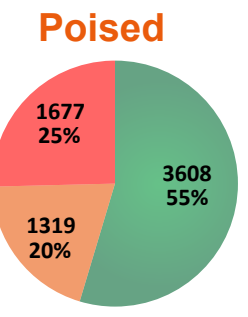

Repressed

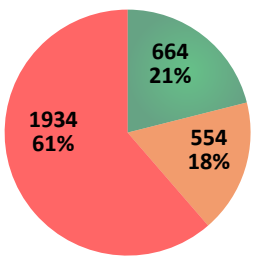

regulation of cell population proliferation 60:0042127

central nervous system development G0:0007417 glial cell differentiation 60:0010001 myelination 60:0042552 glial cell development 60:0021782
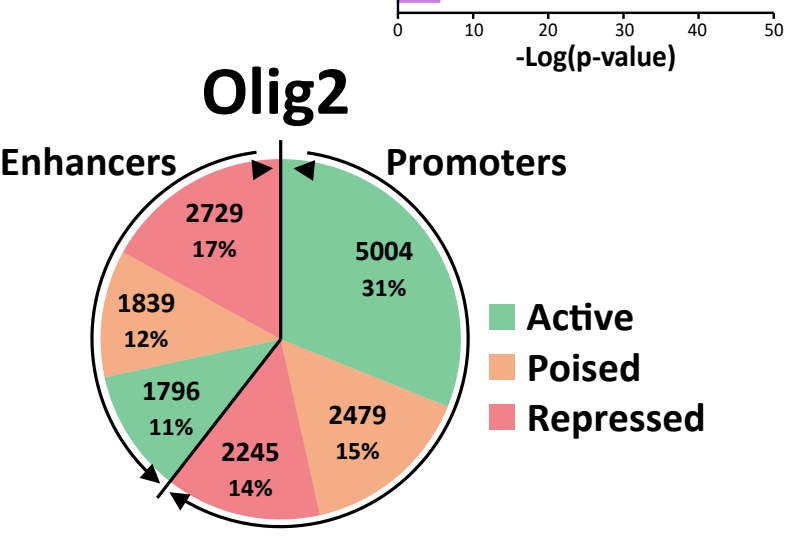

Olig2 alone

Promoters 
Figure S1. Tns3 is a target gene of Olig2 and Chd7/8 regulators of oligodendrocyte differentiation. (A) Scheme representing MACSorting of $\mathrm{O}^{+}$cells from wild type cortices followed by ChIP-seq for histone marks H3K4me3, H3K27Ac, H3K4me1 and H3K27me3. (B) Schematic representing gene regulatory elements in $\mathrm{O}^{+}$cells as active, poised, and repressed, according to their histone marks. (C) Left, diagram representing the classification of gene expression. Data from Marie et al, 2018. Right, Pie chart representing percentage of medium-to-high, low or not expressed genes having an active, poised and repressed promoter in $\mathrm{O}^{+}$cells. Note the high percentage of medium-to-high expressed gene having active marks in their promoter and the high percentage of not expressed genes having repression marks in their promoter. (D) Venn diagram representing the method made to determine Olig2 binding peaks. As the quality of the peaks in replicate 2 was better than in replicate 1, we considered peaks common in both replicate and the peaks of replicate 2 that were present in regulatory elements (regions with histone marks). 16,578 peaks were found corresponding to 8,672 genes bound by Olig2. (E) Gene ontology category found enriched in Olig2 bound genes. (F) Pie chart representing the number and repartition of Olig2 binding sites in regulatory regions with different activity histone marks. Note that Olig2 binds in both promoters (60\%) and enhancers (40\%), and that it is found in either active, poised or repressed regions. (G) Venn diagram representing the amount of Olig2 binding sites which are also bound by Chd7 and/or Chd8 (7,674; Olig2 co-bound) or not (8,894; Olig2 alone). (H-I) Pie chart representing the number and repartition of Olig2 co-bound $\mathbf{( H )}$ or alone $\mathbf{( I ) ~ s i t e s ~ i n ~ r e g u l a t o r y ~}$ regions with different activity histone marks. Note that $42 \%$ Olig2 co-bound sites are in active promoters, whereas $48 \%$ of Olig2 alone sites are in repressed promoter or enhancers. 
Figure S2

A

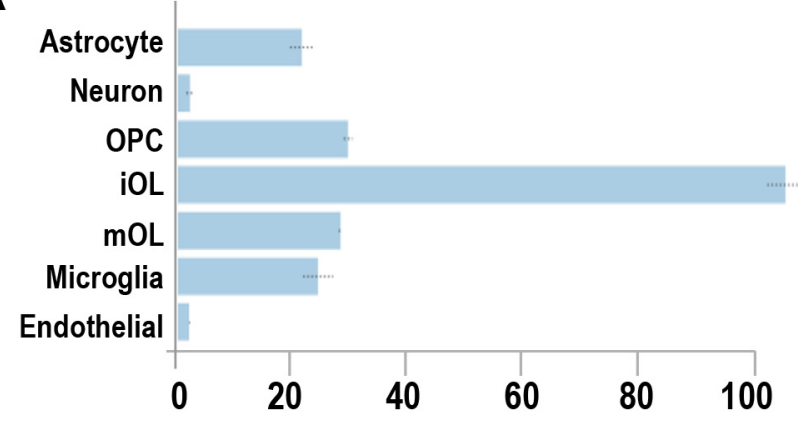

Mouse

B

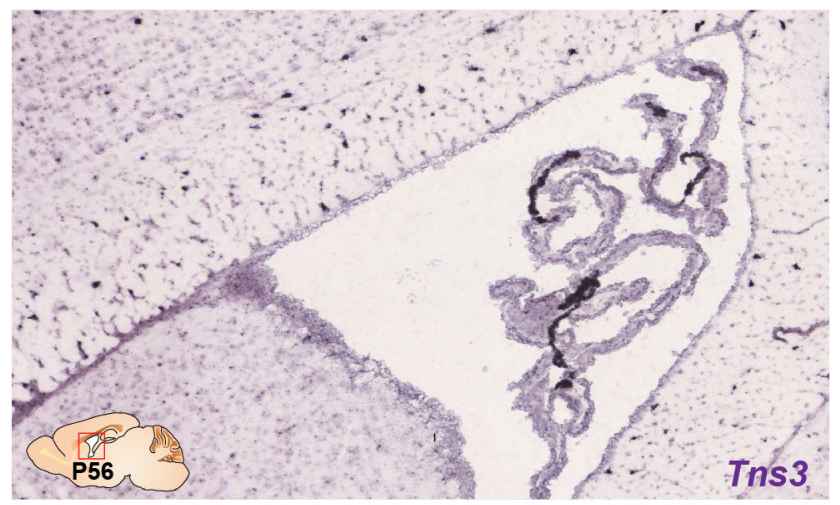

C

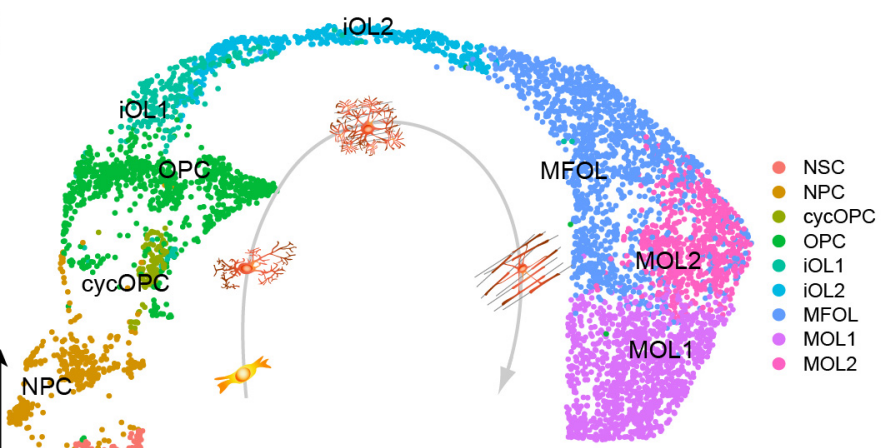

D

Tns3

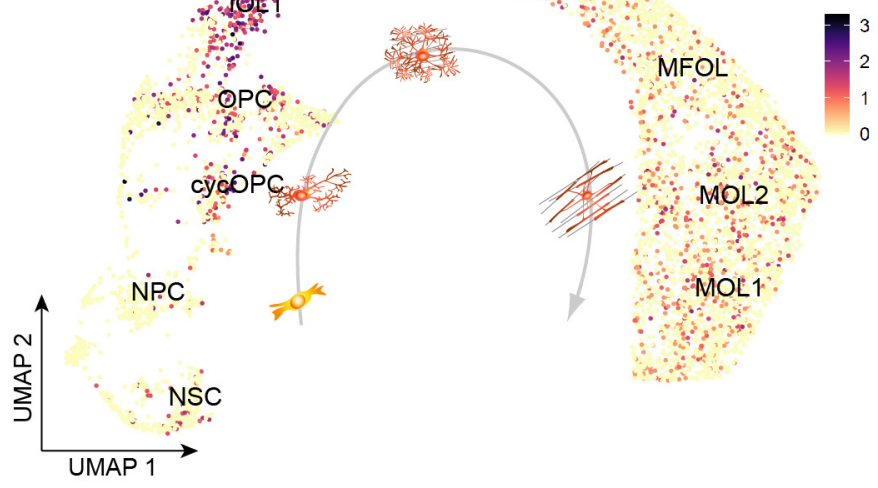

E

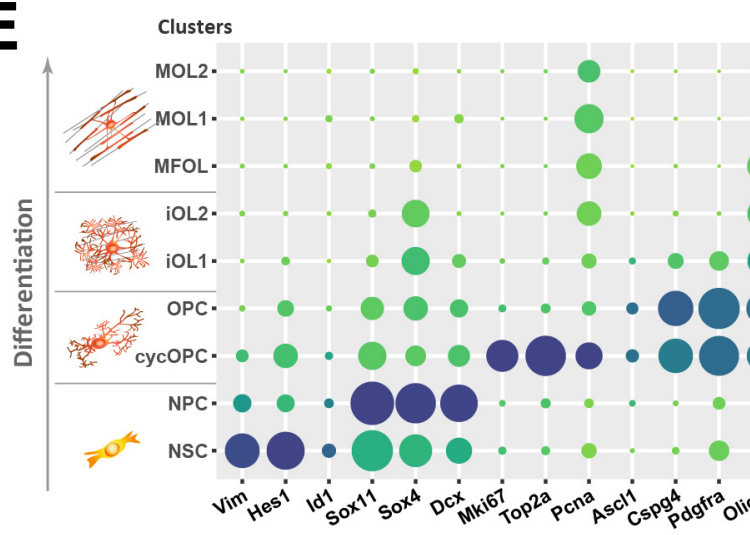

00 UMAP 1

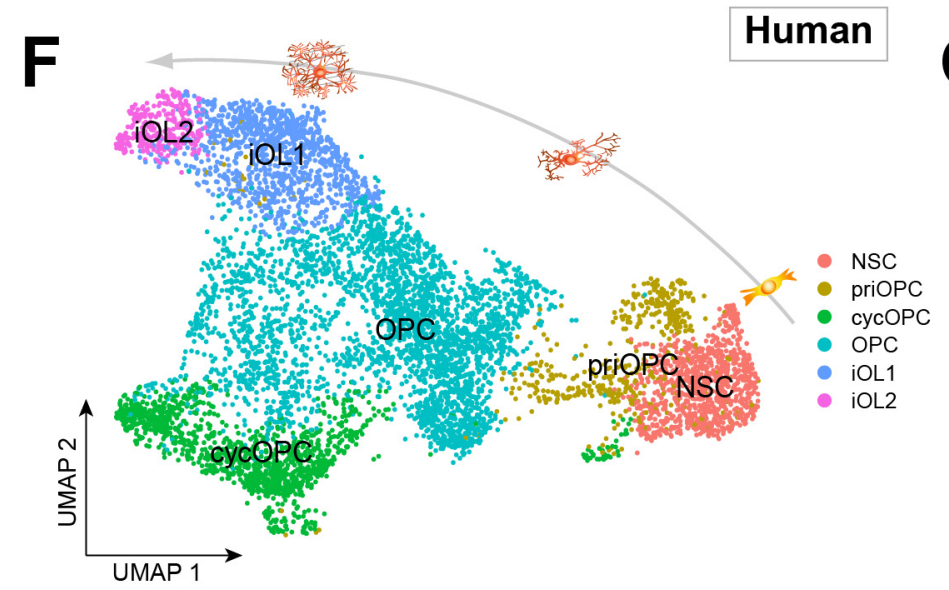

G

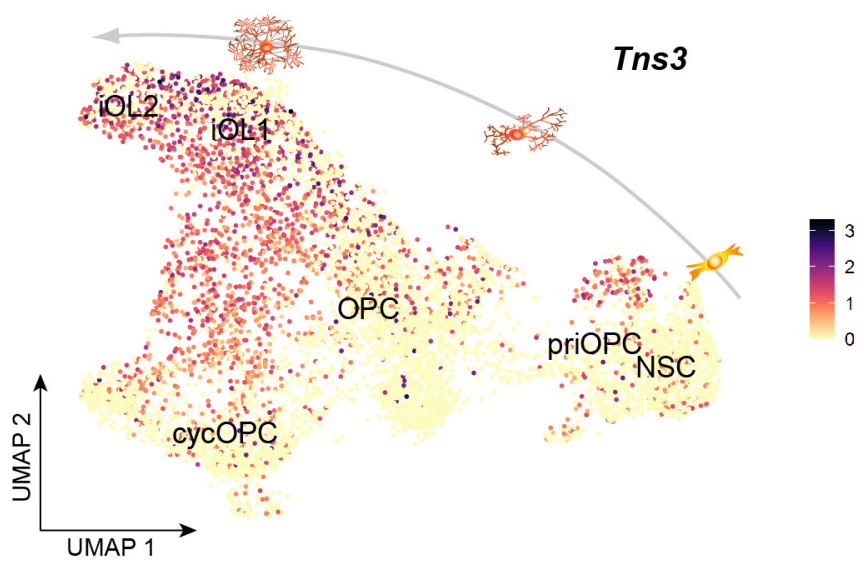

H

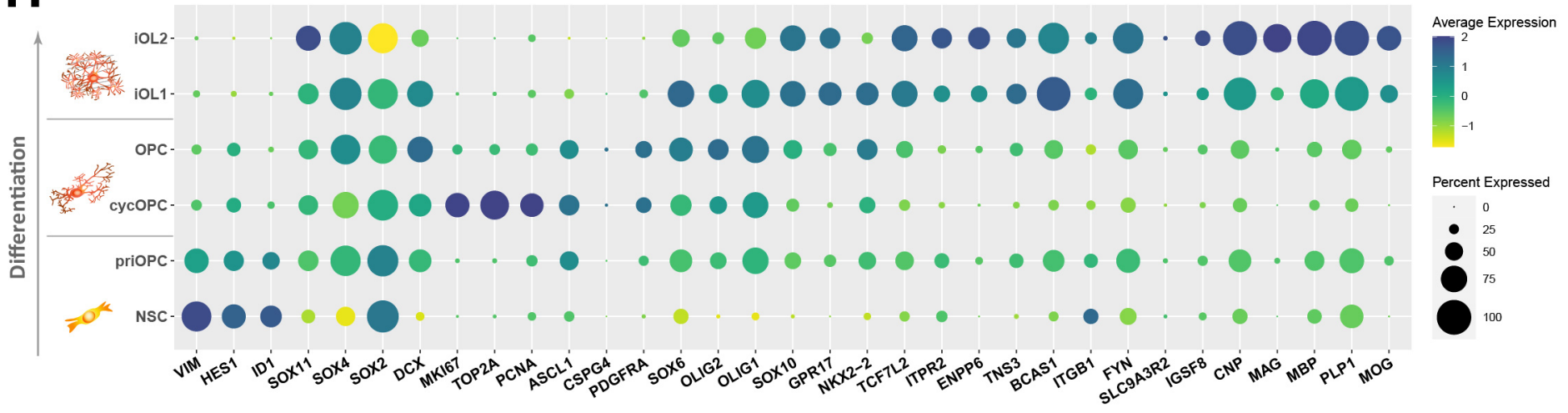


Figure S2: Strong expression of Tns3 transcripts in mouse \& human immature oligodendrocytes. (A) Barplot of Tns3 mRNA transcript levels (FKPM) in postnatal brain cell types (Zhang et al., 2014; brainrnaseq.org). (B) In situ hybridization in sagittal section of the adult (P56) mouse brain at the level of the lateral ventricles showing Tns3 transcript expression in sparse white matter cells (Allen brain atlas, portal.brain-map.org). (C) UMAP representation of neural progenitors and oligodendroglial cells extracted and integrated using Seurat from scRNA-seq datasets (Marques et al., 2016; 2018), representing different clusters corresponding to different oligodendroglial stages of NSCs to mOLs. (D) Feature plot representing relative expression levels of Tns3 transcript. (E) Dot plot representing the transcript expression of key markers for each cell stage/subtype and key oligodendroglial factors in the different clusters showing the predominant expression of Tns3 in iOL1s and iOL2s clusters, similar to Enpp6 and Itpr2. NSC, neural stem cells; NPC, neural progenitor cells; cycOPC, cycling OPC; MFOL, myelin forming OL; MOL1/2, myelinating OL 1/2. (F-H) TNS3 expression in human oligodendroglial cells differentiated from iPSCs by scRNA-seq (Chamling et al., 2021), showing TNS3 high levels of expression in immature oligodendrocytes. (F) UMAP representation showing six clusters (NSC: neural stem cells, cycOPC: cycling OPCs, OPCs, and two iOL stages, iOL1 and iOL2, similar to mouse oligodendroglia). (G) Feature plot showing TNS3 expression levels. (H) Dotplots showing TNS3 expression patter together with key markers of each cluster and regulators of oligodendrogenesis. 
Figure S3
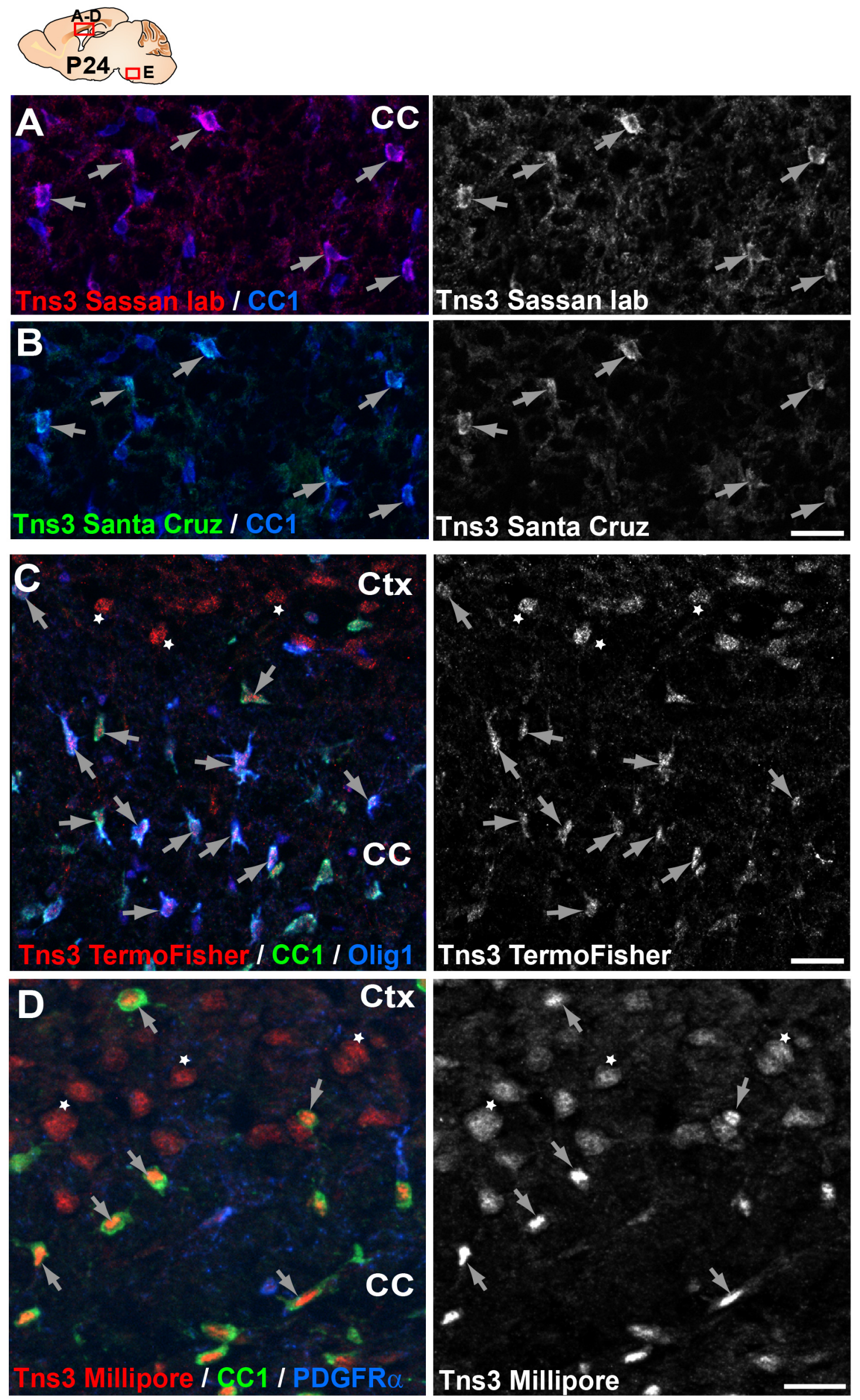
bioRxiv preprint doi: https://doi org/10.1101/2022.02.25.481980; this version posted February 27, 2022. The copyright holder for this

Figure S3: Immunodetection of Tns3 protein in immature oligodendrocytes of the postnatal

brain. Tns3 expression in $\mathrm{CC}^{+}$OLs (arrows) at the level of the corpus callosum (CC) of P24 mice, using a homemade antibody from Sassan Hafizi lab (University of Portsmouth) (A), and commercial antibodies from Santa Cruz (B), ThermoFisher (C), and Millipore (D). Note that Millipore antibody is the only one to show clear nuclear localization in OLs. ThermoFisher and Millipore antibodies also recognize a nuclear signal in cortical neurons (C-D, stars), not found with other Tns3 antibodies. Scale bar, $20 \mu \mathrm{m}$. 


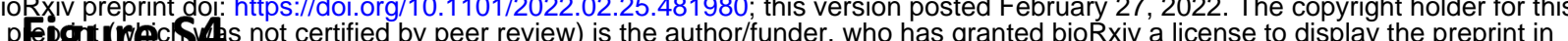

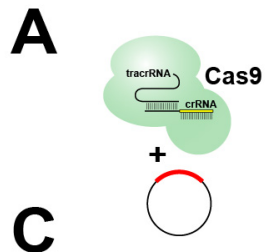
perpetuity.
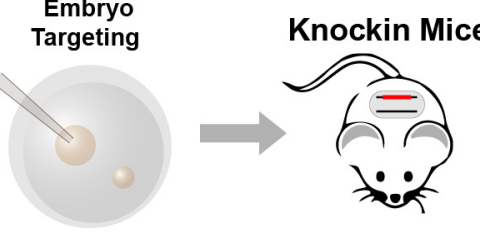

B

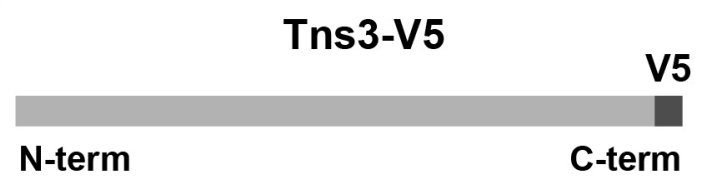

Tns3 wild type allele $3^{\prime}$ region of Tns 3 including the stop codon (TGA) (5'-3')

ACAATCAGAGAAAGCTCTTCTTCCGGAGGCATTACCCGGTCAGCAGTGTGATTTTCTGTGCTTTGGATCCACAAGACAGGAAGTGGATCAAAGATGGCCCTTCTTCT AAAGTCTTTGGATTTGTGGCCCGGAAACAGGGCAGTGCTACAGACAACGTATGCCACCTGTTTGCAGAGCATGA

TGAgccttcctagccaaacctgccagatgcctcatagggccctcaacatggcagtggggtgaggcaggg ccccattcgtaccagattgacaatcttcacattccagactcaacaagaggagaagaatatgacaactcttcaaaccaagaacaaacaatgtcaccactgactggcc ttaaagaagctgctcttccgacacacctatatagtcaggcgcattttgagtggatggatcttggcccgggaaatgcctcatcagggccccaagg

CCCCGAGCAACCTGC 5' homology arm

Tggggtgaggcaggg 3' homology arm

TGA stop codon

cottcctagccaaacctgccaga PAM and gRNA targeted sequence

Targeting sSODN primer for Tns3-V5 tagging (5'-3')

cCCCGAGCAACCTGCCAGTGCCATTGTCAACTTTGTGTCAAAGGTCATGATTGGCTCCCCTAAGAAGATCggtaagcctatcCc

CTt modified PAM

Tns3-V5 tagged allele sequence (5'-3')

ACAATCAGAGAAAGCTCTTCTTCCGGAGGCATTACCCGGTCAGCAGTGTGATTTTCTGTGCTTTGGATCCACAAGACAGGAAGTGGATCAAAGATGGCCCTTCTTCT AAAGTCTTTGGATTTGTGGCCCGGAAACAGGGCAGTGCTACAGACAACGTATGCCACCTGTTTGCAGAGCATGA

ICCCTAAGAAGATCggtaagcctatccctaaccetctcctcggtctcgattctacgTGAgcccttcctagccaaacctgccaga tgcctcatagggccctcaacatggcagtggggtgaggcagggcccccattcgtaccagattgacaatcttcacattccagactcaacaagaggagaagaatatgaca actcttcaaaccaagaacaaacaatgtcaccactgactgqccttaaagaagctgctcttccgacacacctatatagtcaggcgcattttgagtg

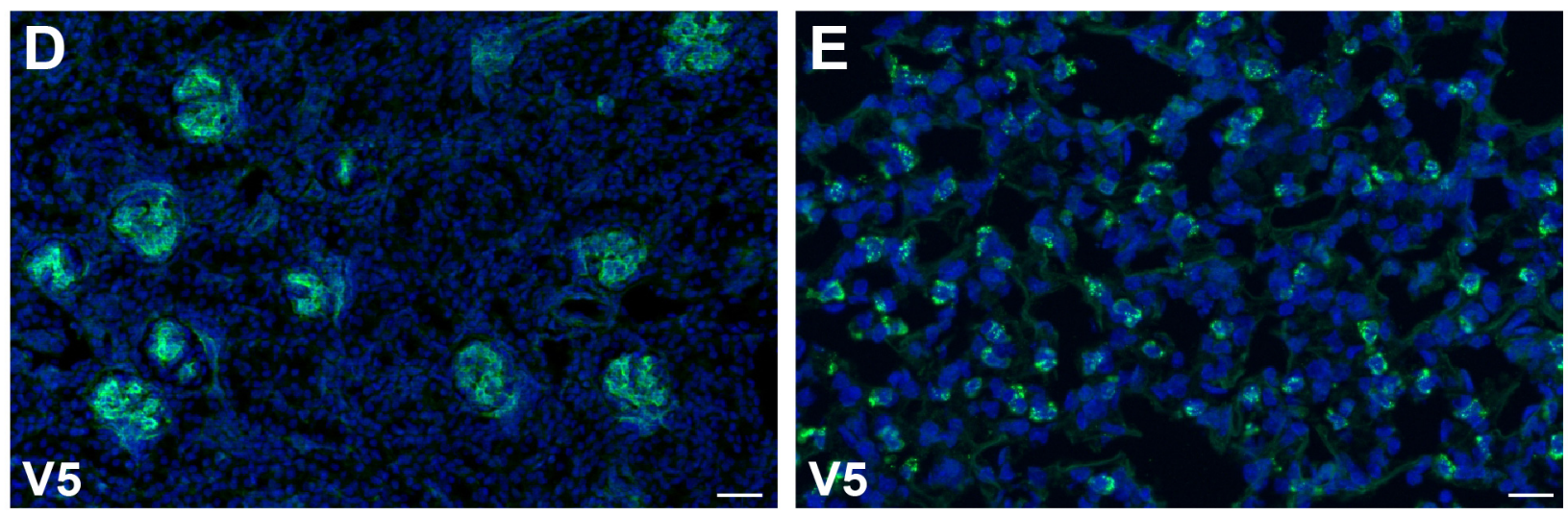

$\mathbf{F}$

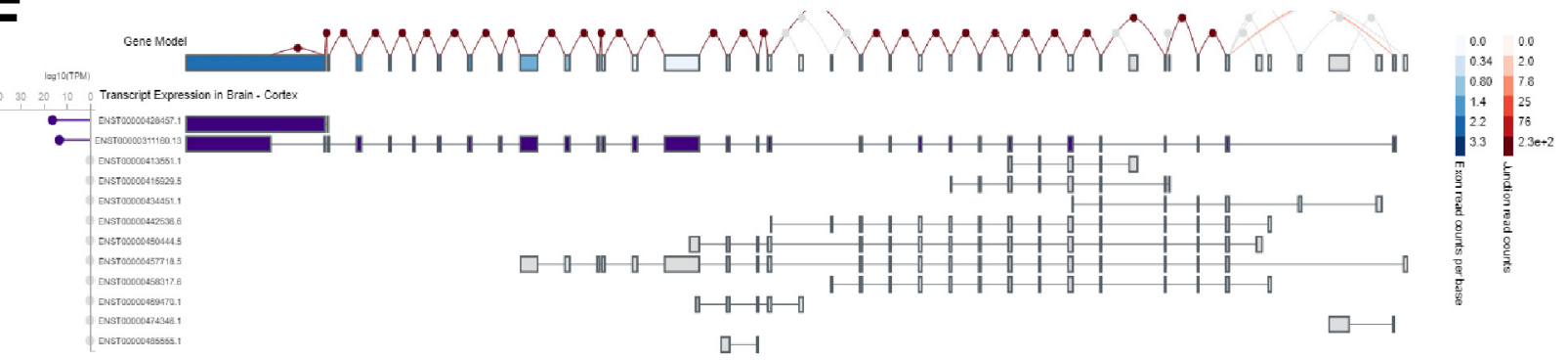

$\mathbf{G}$

\section{MACSorted $\mathrm{O4}^{+}$cells}

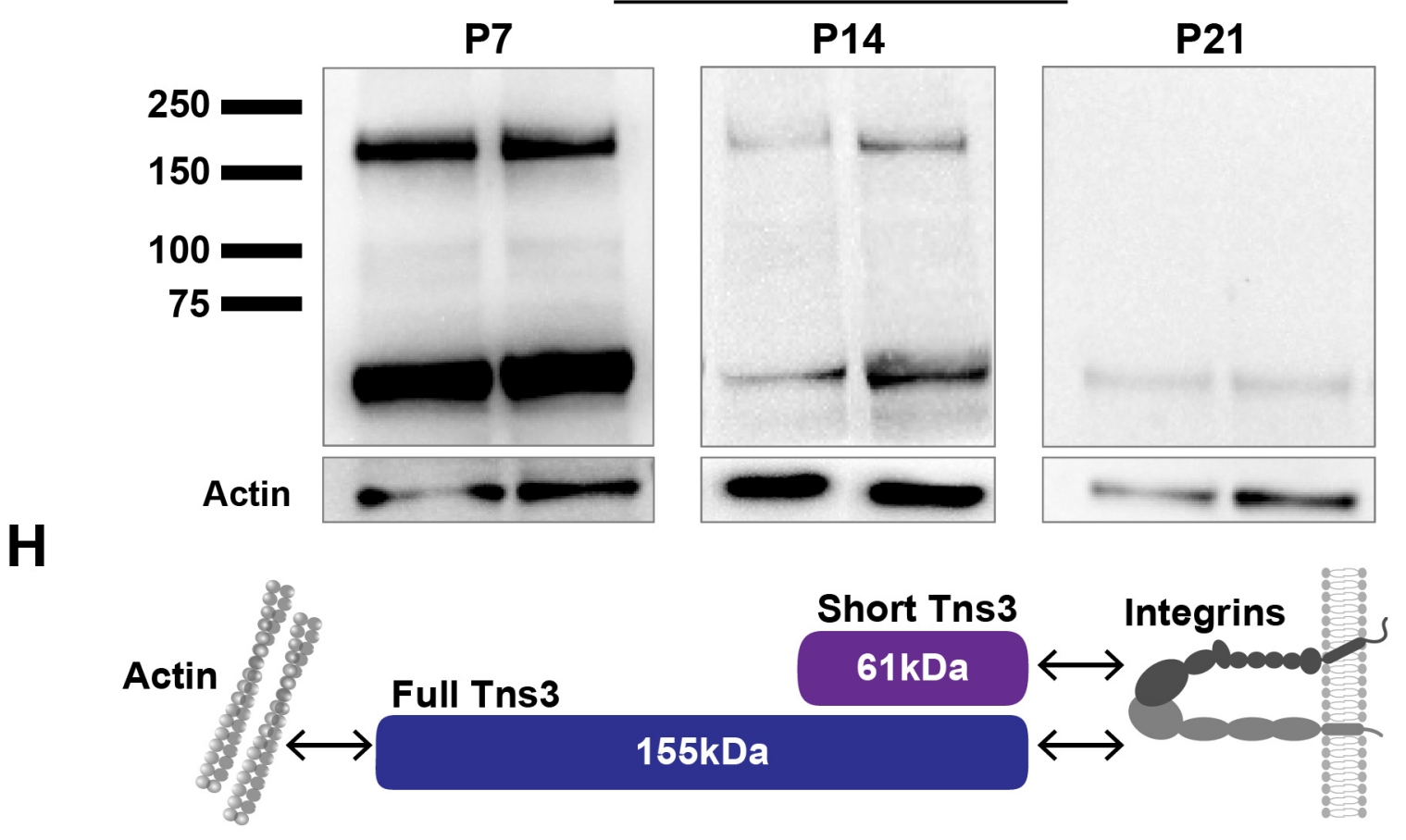


Figure S4: Generation of Tns3 ${ }^{\text {Tns3-V5 }}$ knock-in mice. (A) Scheme of V5 tag knock-in strategy in mice. (B) Schematic of Tns3 protein with a V5 tag at the C-terminal. (C) Nucleotide sequence of a wild type mice Tns3 3' region (Upper), of the targeting ssODN vector (Middle) and of the $3^{\prime}$ region of the first Tns3 ${ }^{\text {Tns3-V5 }}$ knock-in mice generated (Lower). (D,E) Immunostaining using V5 antibody in kidney (D) and lung (E) of P14 Tns $3^{\text {Tns3-V5 }}$ mice. Note the expression of Tns3 in kidney glomeruli and lung alveolar cells (F) Schematic of the two main TNS3 isoforms exon expressed in the human brain (gtexportal.org/home/gene/TNS3). A dark color is associated with higher expression. (G) Western blot of V5 antibody in 04+ MACSorted cells from P7 OPCs/iOLs (left), P14 iOLs (middle) and P21 OLs (right) of Tns3 $3^{\text {Tns3-v5 }}$ mouse brains. Bottom, Actin loading control. Note the presence of the full-length isoform only in OPC/iOLs. (H) Schematic of Tns3 protein brain isoforms and their interactions with actin and integrins. 


\section{Figure S5}
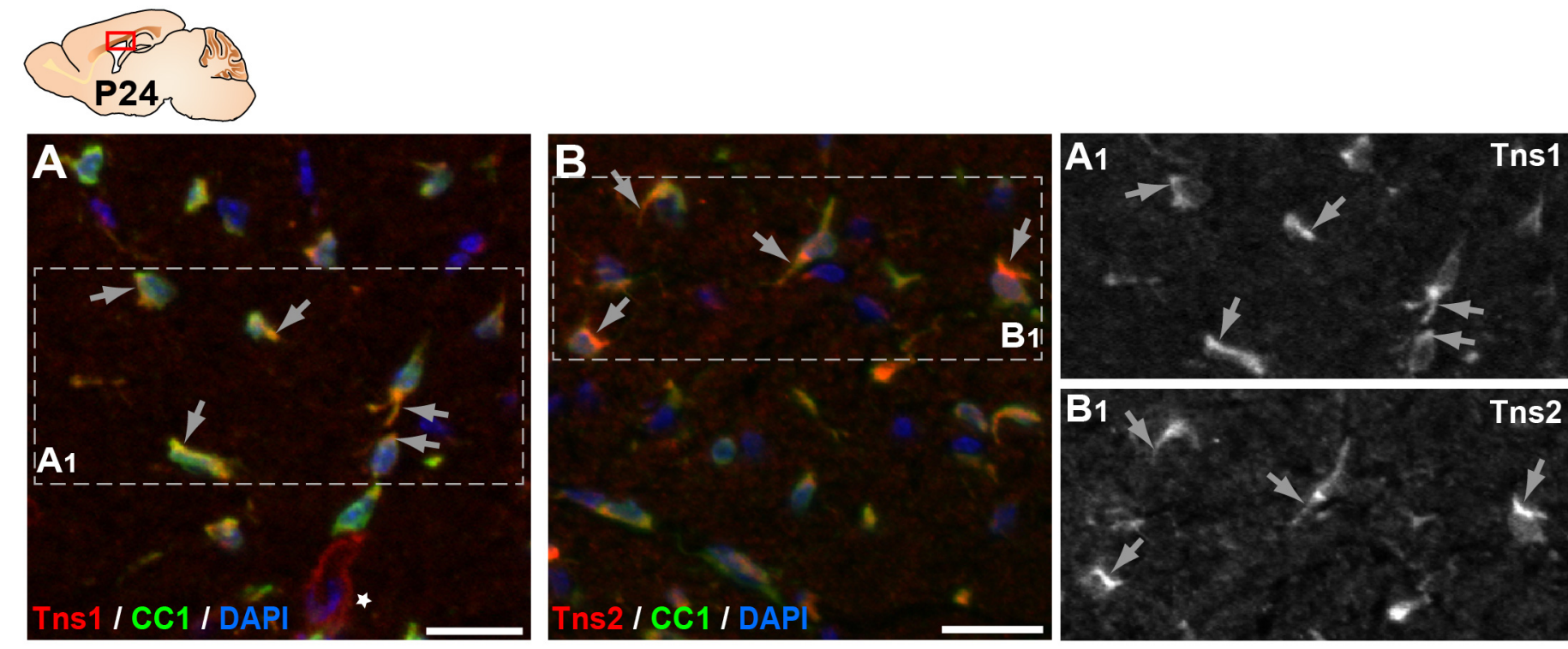

C
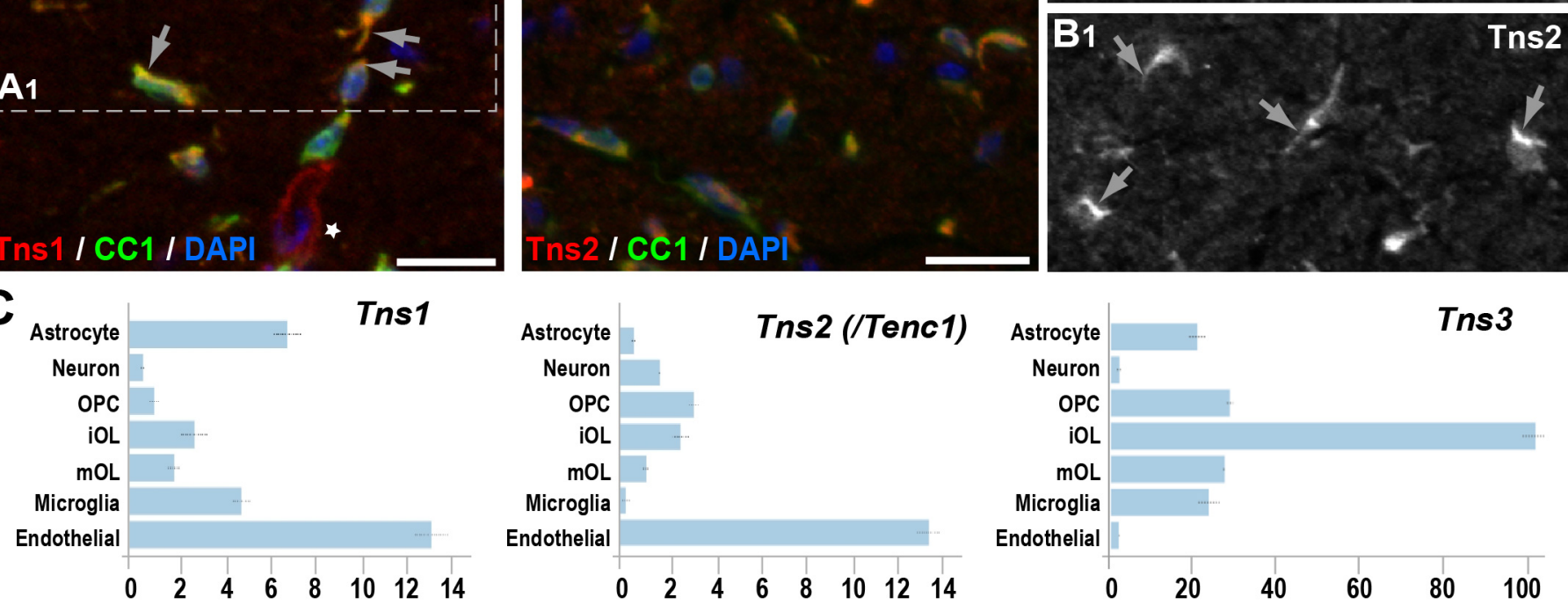
bioRxiv preprint doi: https://doi org/10.1101/2022 02.25.481980; this version posted February 27, 2022. The copyright holder for this preprint (which was not certified by peer review) is the author/funder, who has granted bioRxiv a license to display the preprint in perpetuity. It is made available under aCC-BY-NC-ND 4.0 International license.

Figure S5: Tns1 and Tns2 proteins are detected at low levels in immature oligodendrocytes. Immunofluorescence in sagittal section of P24 mouse brain. Tns1 (A) and Tns2 (B) expression in $\mathrm{CC}^{+}$OLs of the corpus callosum. Tns1 expression is cytoplasmic and excluded from OL nucleus $\left(\mathbf{A}_{1}\right)$, as is Tns2 (B $\left.\mathbf{B}_{1}\right)$. (C) Tns1, Tns2 and Tns3 mRNA expression in postnatal brain (brainrnaseq.org). Note that Tns3 is the main Tensin expressed in oligodendroglia. Scale bar, $20 \mu \mathrm{m}$. 
Figure S6

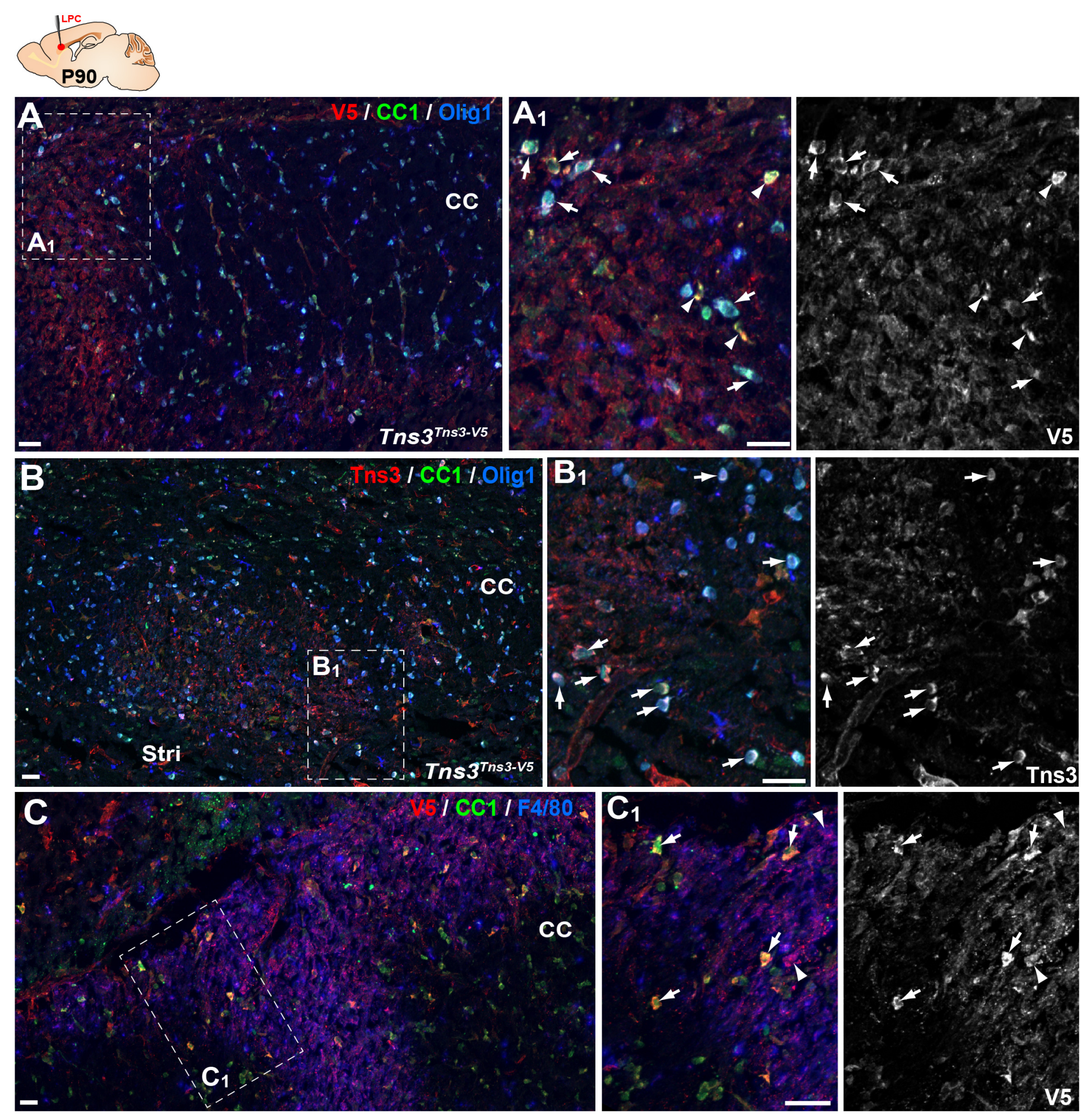

D

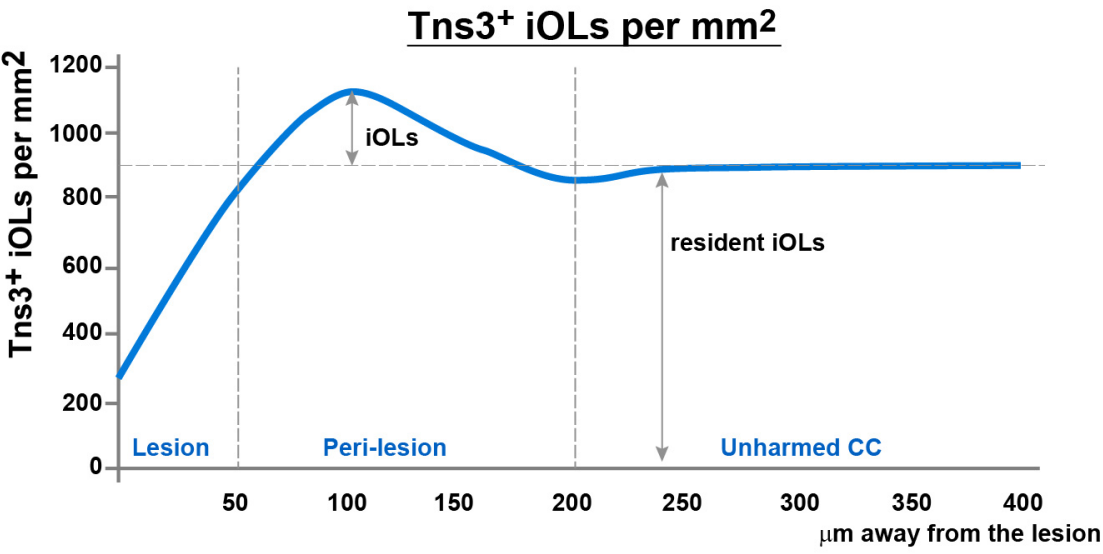


Figure S6: Tns3 is expressed in newly formed oligodendrocytes during adult brain remyelination. (A,B) Tns3-expressing cells in the CC of adult (P90) mice, 7 days after chemically induced demyelination, showing strong Tns3 expression in CC1 ${ }^{+}$Olig1- iOL1s (arrowheads) and $\mathrm{CC1}^{+} / \mathrm{Olig} 1^{\text {cytoplasmic }} \mathrm{iOL2s}$ (arrows) around the lesion using either anti-V5 antibody in Tns ${ }^{\text {Tns3-V5 }}$ mice $\left(\mathbf{A}-\mathbf{A}_{\mathbf{1}}\right)$ or anti-Tns3 antibody in wild type mice $\left(\mathbf{B}-\mathbf{B}_{1}\right)$. Note the absence of $\mathrm{CC}^{+} / \mathrm{Olig}^{+}$OLs in the lesion. (C- $\mathbf{C}_{1}$ ) Tns3 expression in $\mathrm{CC}^{\text {high }}$ iOLs (arrows) around the lesion and in some $\mathrm{F} 4 / 80^{+}$microglia (arrowheads) in the lesion area. $A_{1}, B_{1}$ and $C_{1}$ are higher magnification of corresponding insets (squares). (D) Quantification of the $\mathrm{Tns} 3^{+} \mathrm{OLs}$ density each $50 \mu \mathrm{m}$ distance away from the lesion. Scale bar, $20 \mu \mathrm{m}$. 


\section{Figure S7}

A

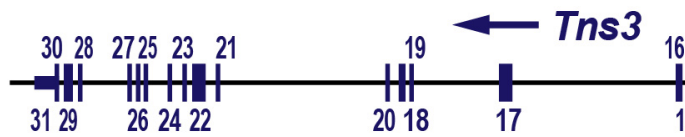

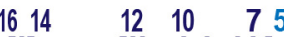

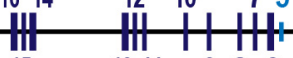

$\begin{array}{lll}1311 & 9 & 8 \\ & 6\end{array}-$ primers

primers

primers -

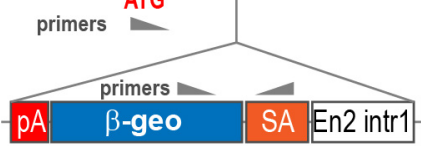

primers

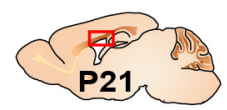

$\beta$ geo insertion
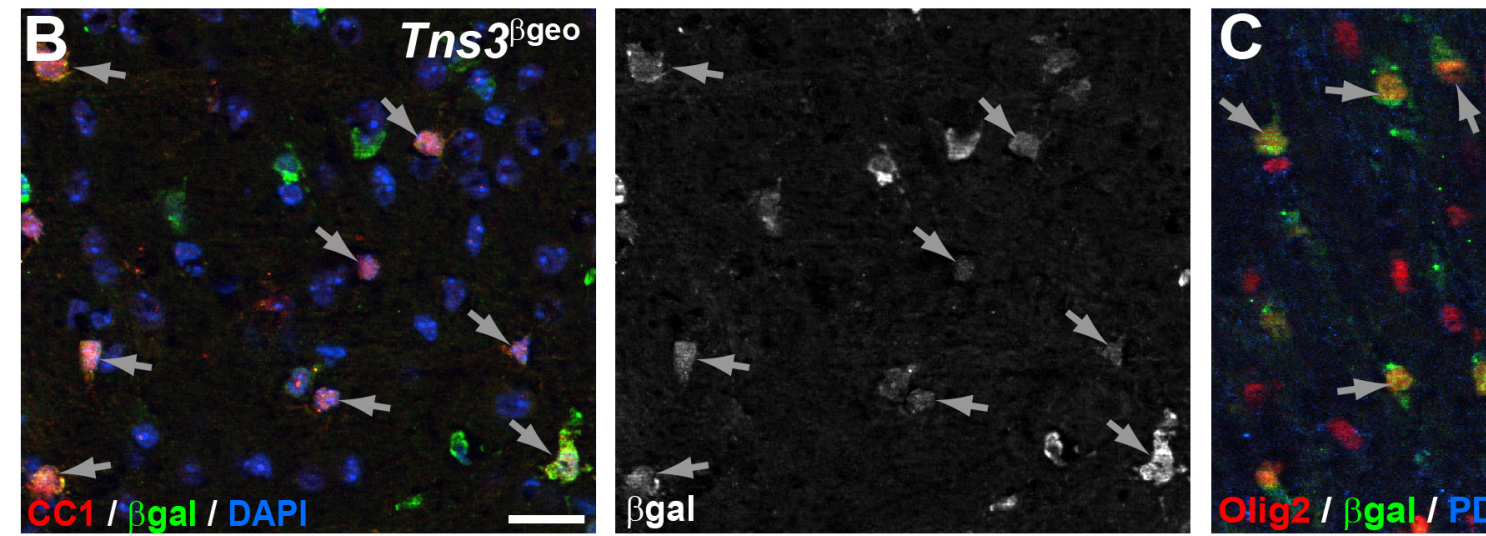

Tns3 $3^{\text {Bgeo }}$

D

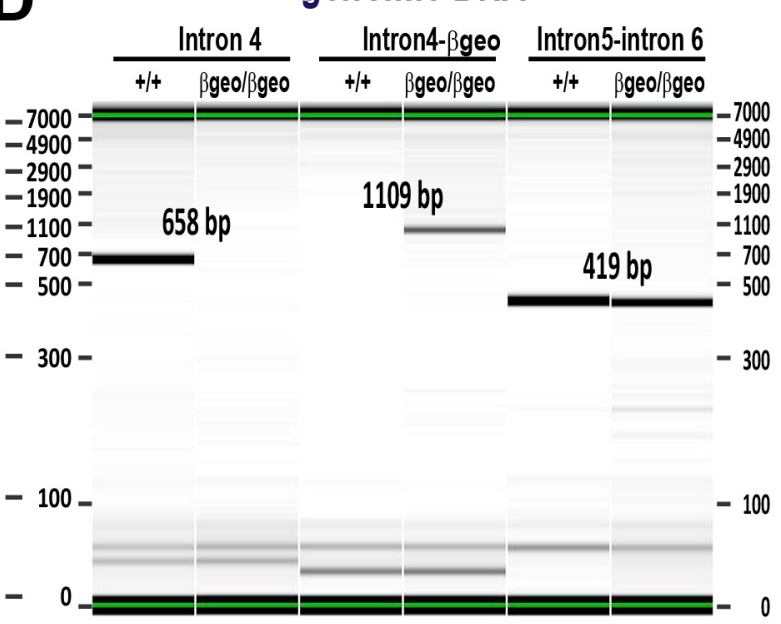

E
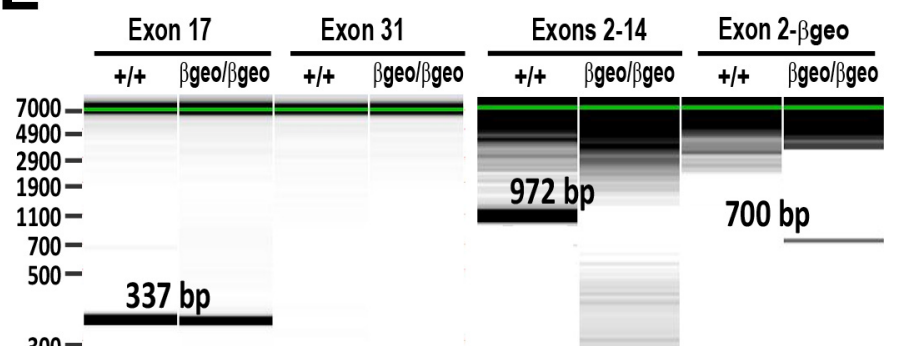

$181 \mathrm{bp}$ non-coding exon coding exon 
Figure S7: Tns $3^{\beta g e o}$ mice still expresses Tns3 full-length transcripts in the brain. (A) Schematic of Tns3 $3^{\beta g e o}$ allele, showing localization of primers used for PCR amplification. $(\mathbf{B}, \mathbf{C})$ $\beta$-galactosidase immunofluorescence in P21 sagittal brain sections, correspond to $\mathrm{CC}^{+} \mathrm{OLS}$ (B) or Olig2 $2^{\text {high }} /$ PDGFR $\alpha^{-}$OLs (C) immature OLs. (D) PCR from genomic DNA showing the presence of the native Intron 4 only in wild type $(+/+)$ mice, of the Intron $4-\beta g e o$ only in

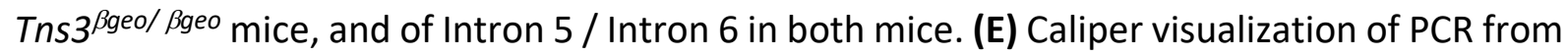
cDNA showing the presence of Exon17 and Exon31 both in Tns3 $3^{\beta g e o / \beta g e o}$ and wild type mice, despite the amplification with primers for Exons 2-14 only in wild type mice, and with primers for Exon2- $\beta$ geo only in Tns $3^{\beta g e o / \beta g e o}$ mice. CC, corpus callosum. Scale bar, $20 \mu \mathrm{m}$. 


\section{Figure S8}

A

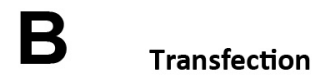

Selection of

transfected cells Amplification DNA extraction

SgRNA ${ }_{\text {Cas9 }}^{\text {Tol2 puroR }}{ }^{\text {pcMv }}$

Integrative CRISPR/Cas9 vector

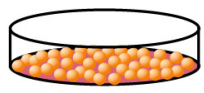

cell line

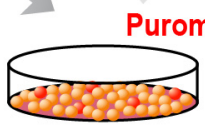

2 days

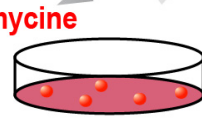

3-5 days

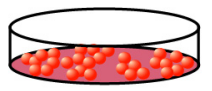

3-5 days mutation detention

C

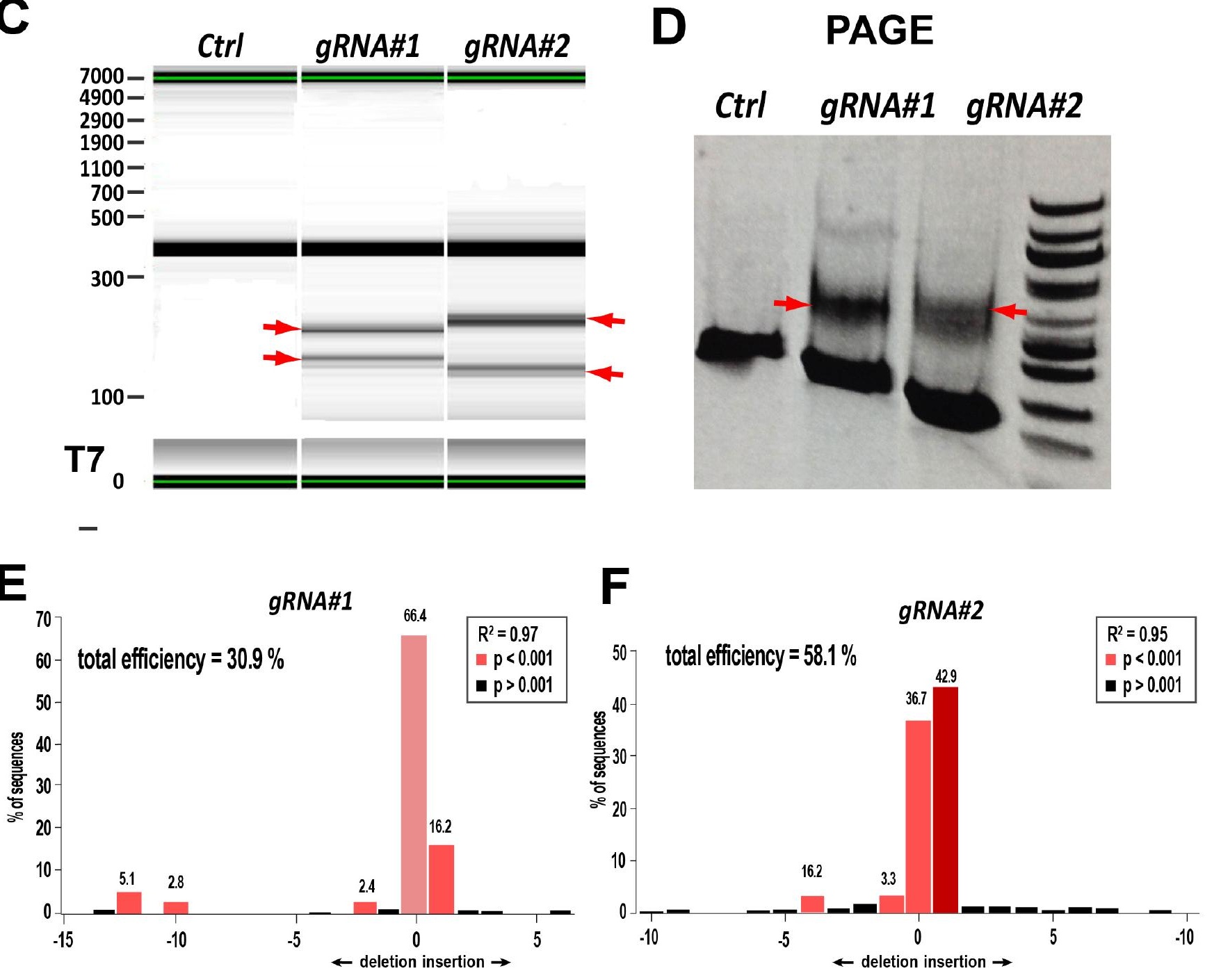

들
들 
Figure S8: Generation and validation of Tns3-targeting CRISPR/Cas9 tools. (A) Schematic of the Tol2-PX459 CRISPR/Cas9 expression vector allowing Tol2-DNA integration driving Cas9 \& puromycin resistance expression (from polycistronic $2 \mathrm{~A}$-mediated cleaved) driven by CMV promoter and sgRNA expression from U6 promoter. (B) Scheme of C17.1 neuroblastoma cell line lipofection followed by puromycin selection of transfected cells used to amplify by PCR the Tns3 targeted region and assess for indel mutations. (C) Caliper visualization of PCR products obtained after T7-mediated cleavage of DNA heteroduplex due to the indel mutations, showing smaller products (arrows) generated by Cas9 cutting with gRNA\#1 or gRNA\#2. (D). Visualization Tns3-targetted region amplified by PCR run in a PAGE gel. Note the extra bands in gRNA\#1 or gRNA\#2 transfected cells corresponding to Cas9 cut products, contrary to Tol2-PX459 empty plasmid (control). (E,F) TIDE analysis representing the percentage of sequences presenting a given indel mutation in cells transfected with gRNA\#1 (E) or gRNA\#2 (F). 


\section{Figure S9}

A

TNS3 tensin 3

Category Expected SNVs Observed SNVs Constraint metrics

\begin{tabular}{|c|c|c|c|c|}
\hline Synonymous & 378.8 & 409 & $\begin{array}{l}Z=-1.22 \\
o / e=1.08(0.99-1.17)\end{array}$ & 0 \\
\hline Missense & 863.1 & 785 & $\begin{array}{l}Z=0.94 \\
o / e=0.91(0.86-0.96)\end{array}$ & $0 \_0$ \\
\hline pLoF & 61.7. & 6 & $\begin{array}{l}\mathrm{pLI}=1 \\
\text { o/e }=0.1(0.05-0.19\end{array}$ & $0 \underline{0} 1$ \\
\hline
\end{tabular}

Constraint metrics based on Ensembl canonical transcript (ENSTO0000398879.1).

C

CHD7 chromodomain helicase DNA binding protein 7

Category Expected SNVs Observed SNVs Constraint metrics

\begin{tabular}{|c|c|c|c|c|}
\hline Synonymous & 611.6 & 637 & $\begin{array}{l}Z=-0.81 \\
o / e=1.04(0.97 .-1.11)\end{array}$ & $0 \_\triangleright_{1}$ \\
\hline Missense & 1618.5 & 1254 & $\begin{array}{l}Z=3.22 \\
o / e=0.77(0.74-0.81)\end{array}$ & 0 으 1 \\
\hline pLoF & 137.8 & 5 & $\begin{array}{l}\mathrm{pLI}=1 \\
\mathrm{o} / \mathrm{e}=0.04(0.02-0.08\end{array}$ & 00 \\
\hline
\end{tabular}

Constraint metrics based on Ensembl canonical transcript (ENST00000423902.2).

E

NKX2-2 NK2 homeobox 2

Category Expected SNVs observed SNVs Constraint metrics

\begin{tabular}{|c|c|c|c|c|}
\hline Synonymous & 73.9 & 100 & $\begin{array}{l}Z=-2.38 \\
o / e=1.35(1.15-1.6)\end{array}$ & $0 \longrightarrow \triangleright_{1}$ \\
\hline Missense & .161 .1 & 161 & $\begin{array}{l}Z=0 \\
o / e=1(0.88-1.14)\end{array}$ & 0 \\
\hline pLoF & 9.5 & 2 & $\begin{array}{l}\mathrm{pLI}=0.41 . \\
\mathrm{o} / \mathrm{e}=0.21 .(0.09-0.67)\end{array}$ & $0 \underline{0} 1$ \\
\hline
\end{tabular}

Constraint metrics based on Ensembl canonical transcript (ENSTO0000377142.4).
B

SOX10 SRY-box transcription factor 10

Category Expected.SNVs Obseryed SNVs Constraint metrics

\begin{tabular}{|c|c|c|c|c|}
\hline Synonymous & 145.5 & 130 & $\begin{array}{l}Z=1.01 \\
o / e=0.89(0.77 .-1.03)\end{array}$ & 0 \\
\hline Missense & 301.1 & 166 & $\begin{array}{l}Z=2.77 . \\
o / e=0.55(0.48-0.63)\end{array}$ & 0 \\
\hline pLoF & 14.3 & 0 & $\begin{array}{l}\mathrm{pLI}=0.99 \\
\mathrm{o} / \mathrm{e}=\underline{0}(0-0.21)\end{array}$ & 00 \\
\hline
\end{tabular}

Constraint metrics based on Ensembl canonical transcript (ENST00000396884.2).

D

CHD8 chromodomain helicase DNA binding protein 8 Category Expected.SNVs observed.SNVs Constraint metrics

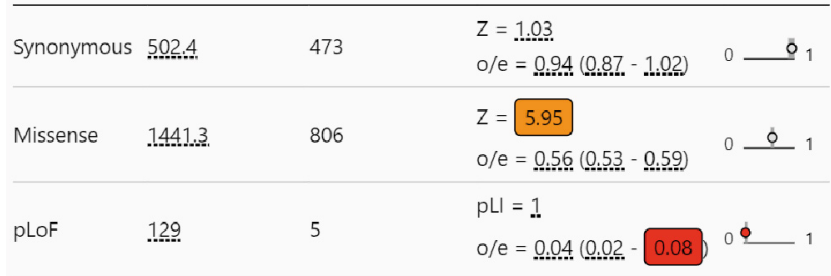

Constraint metrics based on Ensembl canonical transcript (ENSTO0000399982.2).

$\mathbf{F}$

OLIG1 oligodendrocyte transcription factor 1

Category Expected_SNVs observed SNVs Constraint metrics

\begin{tabular}{|c|c|c|c|c|}
\hline Synonymous & 46.6 & 45 & $\begin{array}{l}Z=0.18 \\
o / e=0.97 .(0.76-1.24)\end{array}$ & 0 \\
\hline Missense & 91.2 & 53 & $\begin{array}{l}Z=1.42 \\
o / e=0.58(0.47 .-0.73)\end{array}$ & $0 \underline{0} 1$ \\
\hline pLoF & 3.5 & 0 & $\begin{array}{l}\mathrm{pLI}=0.66 \\
\mathrm{o} / \mathrm{e}=\underline{0}(\underline{0}-0.85\end{array}$ & 00 \\
\hline
\end{tabular}

Constraint metrics based on Ensembl canonical transcript (ENSTO0000382348.1). 
Figure S9: Intolerance for TNS3 loss-of-function variants in the human population. (A-F) GnomAD data for TNS3 and key regulators of oligodendrogenesis (SOX10, CHD7, CHD8, NKX21 , and OLIG1) showing frequency and scores of different genetic variants in the human population: synonymous, missense, and pLOF (nonsense, splice acceptor, and splice donor variants). The numbers highlighted colored squares correspond to the LOEF (loss-of-function observed/expected upper bound fraction), that it is a conservative estimate of the observed/expected ratio. Low LOEUF scores indicate strong selection against predicted lossof-function (pLoF) variation in a given gene, while high LOEUF scores suggest a relatively higher tolerance to inactivation. Note that TNS3, like SOX10, CHD7, and CHD8 have very low LOEUF scores indicating high intolerance for their inactivation, contrary to NKX2-1 and OLIG1, which are show more tolerance to their inactivation. 


\section{Figure S10}

A

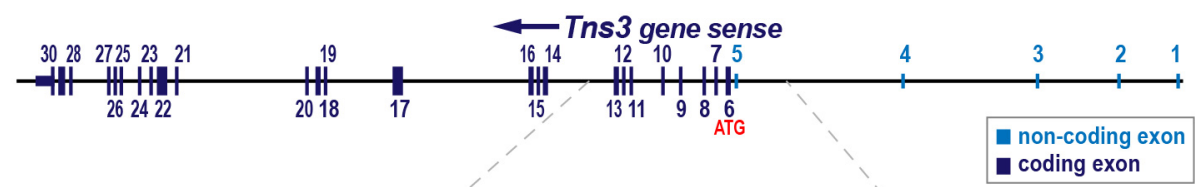

B

Tns3 $3^{\text {Flox }}$ allele

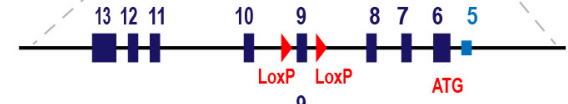

Targeting construct $\underset{\text { LoxP }}{\stackrel{\text { Toupp }}{\text { LoxP }}} \stackrel{9}{\text { Trobp }}$

ATG

89

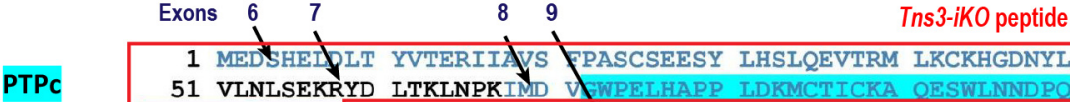

Tyr phosphatase 101 HVWVIHCRGG KGRIGVVISS YMHFTNVSAS ADOATDRFAM KKFYDDKTS

domain 151 IMEPSQKRYV QFLSGLLSGA MKMNTSPLEL HFVIMHGVPS FDTGGACRPF

PTEN C2 201 LKLYOAMOPV YTSGIYNVGS ENPSRIRIAI EPAQLLKGDI MVKCYHKKFR

251 SATRDVIFRL QFHTGAVQGY GLLFGKEELD SACKDDRFPD YGKIELVFSA

301 TPEKIQGSEH LYSDQGVTVD YNTADPLIRW DSYENMSADG EVLHTQGPVD

351 GSLYAKVRKK SASDTGIPSS PQGMPATSSP DHGDHTLSVS SDSGHSTASA

401 RTDKTEERLT PGARRGLSPQ EKAELDQLLS GFGLEDSASS HKDMTDMRSK

451 YSGTRHVVPA QVHVNGDAAL KDRETDILDD EMPHHDLHSV DSLGTLSSSE

501 GPQSTHLGPF TCLKSSQNSL LSDGFGNGVA EDHNGVLSPD LGLGVDTLYD

551 RERMCGGREQ KPLQPLLRKP SAPTPVQAYG QSNYSTQTWV RQQQMVAAHQ

601 YSFASDGEAR LGSRSTVDNT GLAOPPPHIP VTPNRGASSR VAVQRGISNG

651 PNPPDTQQLC PGKALQPRFQ DDRVTNGVHQ EPNTGSSPGS PTLDIDQSIE

$\begin{array}{clll}\text { ABD } & 701 & \text { QLNRLILELD } & \text { PTFEPIPTHL NALGISAVCP DGVGSGLRCS GRIDSVDGPG } \\ \text { An binding } & 751 \text { RSPGRQGDDP IGGRLRKLSI GQYDNDAASQ VTESKCGWGK AGVDPAPSLG }\end{array}$

Actin binding 801 SFSSPEDIKE TVITAYPSDL NMIDGRIPNS KESSMCLTPS FPVSPETPY

851 KTSPRYPPFS PPEPQLSSPA SLHKGREPRG CPEIISHTVG MSESPVGPKP

901 TMLRADMPAT PNFQQVFASS CTVSSNGPGQ RRESPPSAER QWVESSPKST

951 LTLLGNSHPS ESPLGTHEFC SSGKDSPGLP CFOSSELQAS FHSHELSMSE

1001 PQGALPPAGS QTFLGFNTVT TATSVLPPGE DAGTLLVNSH GTSPAPGTPI

1051 LTTGAADNGF LPHNFLTVSP GASSHHSPGL QNQNVSLPGQ PPLPEKKRAS

1101 EGDRSLGSVS PSSSGFSSPH SGSTMSIPFP NVLPDFCRPS EVASPLPDSP

$\begin{array}{llll}1101 & \text { EGDRSLGSVS } & \text { PSSSGFSSPH } & \text { SGSTMSIPFP NVLPDFCKPS EVASPLPDSP } \\ \text { SH2 } & 1151 \text { NDKLVIVKFV ODISKFWYKA DISREOAIAM LKDKAPGSFI VRDSHSERG }\end{array}$

Signal

transduction

PBT

Signal

transduction

FSLWALV OHSITPLATP CKLIIPHRDP LEEIAENSPQ TAANSAAELI

1301 KQGA ACNVWY LNSVEMESLT GHOAVOKALS MTLVOEPPPV STVVHEKVSZ

1351 QGITLTDNQR KLFFRRHYPV SSVIFCALDP QDRKWIKDGP SSKVFGFVAR

1401 KQGSATDNVC HLFAEHDPEQ RASAIVNEVS KVMIGSPKKI

\section{Tns3-iKO peptide}

1 MEDSHELDLT YVTERIIAVS FRASCSEESY LHSLOEVTRM LKCKHGDNYL 51 VLNLSEKRYD LTKLNPKIMD VGWPELHAPP IDKMCTICKA QESWLNNDPQ 101 HVVVIHCRC*

\section{Tns3 floxed targeting construct sequence}

gat aTCAGTTGTAAAGTTCCCTCTTGTGTGCACAAGGTTTGACTCTAGTACGGACTCTTGGTGAGCTACAAAGCAGTGACCTGCCAGTTPGGCCTTTGTATGGCTTGG

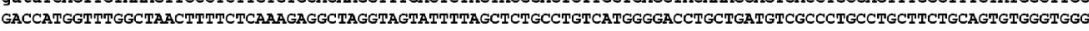
GGCTGCTGGAACAGATCAGCCAGTCTCCAGGCAGACGATAAGATGATGATGGTTAAGTITTGTGTGACTTTCACATCTCACAGACTAGTTCTGTTTTGATTTTTTTT TTTTTTGCAATGCTTAAAATATGTGATAATGTGTTTACAGATTGTAGGAAAAGAGTAGGTGGTCAGAATGGAACTGGCTTTGGGTTACCGTTTGCAGAGCCCTGTTA GTAGGTCACAACAGTGTGTTCTGAAGTTTCAGACCAGTACATGGTTCTCTGTGAGGCTGCACTGCCCCACAGAGCAGTAGAGGAAGCTCTACTATTGGTGATGTTGA CTGTGAATATGGCTGTGTTTAGGAGGATTTCCATAGCACCAGCCTGGGATGTGGTTTCCATGTGTATAACCCCCCCCTCCCCCCACACACACACACATACAGTGAAT AAAGTGTGAGGTTTGGCACCAGTGTAGGATACACCAGTT TACCAGGTTCCGAGGTCCTGCCACTTAGAGCCGTATACACAGACGGCTGTCACTTGTCAGCCCCTGGG CAGACATTCATTCCTGTITCACctcgagATAACTTCGTATAGCATACATTATACGAAGTPATgtcgaCGTGGTGAGTGACTGGCTGCTGCCGTGTGGATATGCCCTC CGGGGACTTACACAGACACCCAGCTAGCACCTGCTCTTGTTATGGGCCCCTGTGTGAAGAGTCTCTGCTTAACTCTGGTGCTCTAATGTVTAGGGAGGCAGAGGGGTT AATACTTGTTCTCTGCTACTCTGTTCATGATTGAGCATGCTCCTGCCTCATTCCGTCGGTATATAGGATTCTATAGAAAGAACATTGGTPTCTCTCTGGTCTCCTGC AGGGTGGTAAAGGACGCATTGGAGTGGTCATATCGTCCTACATGCACTTCACCAACGTCTCAGCCAGGTAAGATGGAGAGGGGGCTCATPTCCACGGGGTGTTGGAA GTTGGTTCACGCATGAGGTCTICCTGGGAGTCATCTTGACCTGACTTGTCCCTPAGTGCCAGAGTGTTGTGTGCCTAGTCTCATTGTCCAGCAGGATGGGAAAGATA TATTCTTGTTTGGGCTGTTCTGTGTGACTGATTCCCAGGAGCTCTATGGAGGCAAGCAGACTAAGTGAGCCCAGTTATTCTCTATGAAAACAGTCGTGTTGATGTCA cgtcgacATAACTTCGTATAGCATACATTATACGAAGTTATctcgagTACACCTTGTAAGTTTAATAAAAACAGCCATCATGTTCTGTTTGTTCTGAAAGTCCACTG TATGTTATCTCTAAGATGATAGGACCCTGGTCTGTGCCCAGCCATTGTGGCAATGGACAGTCAGGGTGTCTCCTGATGTGCTTTCTGAAGAAAGGCCAAATTTCAG TTTGGAAAATGTCATTGTTGGGTAGGAGTATAGTTTTGTTTAATGCACTTGCCTAGTGGGTGCAAGACCTCAGCATCCTTCCCAACCCTGCACATGAAAATCATTTG CACGCTCCTGGGTGCAGGAGCTGTATGCCCAGGCCAGTCATTTGCACACTCCTGGTCGTGCAGGAGCTGTATGCCCAGGCCAGTCACTIGCACACTCCTGGTTGTGC AGGAGCTGTATGGCCAGGCCAGTCACTTGCACACTCCTGGTTGTGCAGGAGCTGTATGCCCAGGCCAGTCACTTGCACACTCCTGGTTGTGCAGGAGCTGTATGCCC AGGCCAGTCATTTGCACACTCCTGGTTGTGCAGGAGCTGTATGCCCAGGCCAGTCACTTGCACACTCCTGGTCGTGCAGGAGCTGTATGCCCAGGCCAGTCATTTGC ACACTCCTGGTTGTCTCAGGAGCTGCGCTGTGTTTTGTGCCCAGGCCTITCCATCCTAAGGAAATAGGAGCCTTCTCAGCTCCCTTATGAGCCTGCTCCTCTGCTGT GTGAAGGAGTGAAATTGGACIPTITGGAATGTTITCTCTGGAAACAAATCAAGAtatC 
Figure S10: Generation of Tns3 floxed allele. (A) Schematic of Tns3 locus, Tns $3^{\text {Flox }}$ allele and targeting vector with LoxP sites flanking Exon9, used to induce homologous recombination in mouse ESCs. (B) Full-length Tns3 amino-acid sequence indicating its protein domains. Squared in red highlights the 109 amino-acid sequence of the Tns3 peptide produced upon Cremediated recombination in Tns $3^{\text {flox }}$ expressing cells. (C) Peptide sequence of protein product predicted for Tns3-iKO expressing cells. (D) Tns $3^{\text {Flox }}$ targeting vector sequence used for homologous recombination in mouse ESCs and generation of Tns $3^{\text {Flox }}$ mice, with LoxP sites highlighted in blue and Exon 9 highlighted in red. 


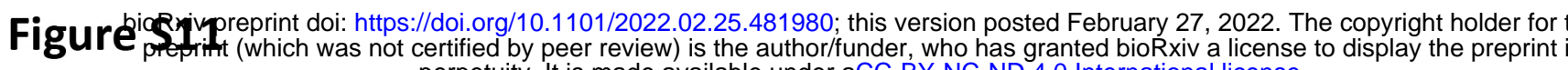

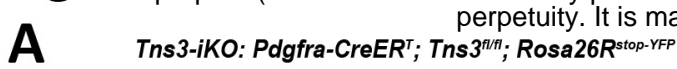
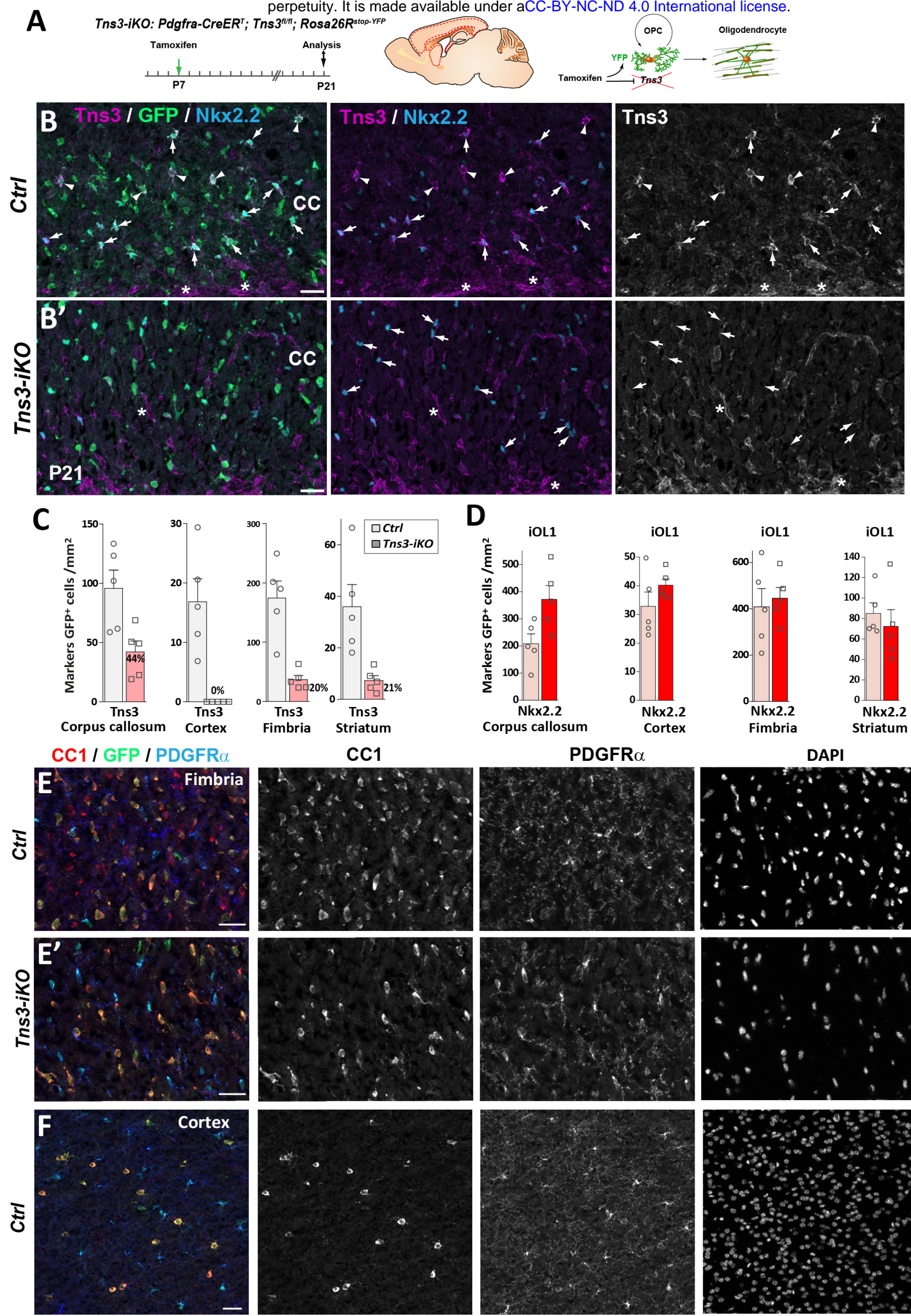

곤

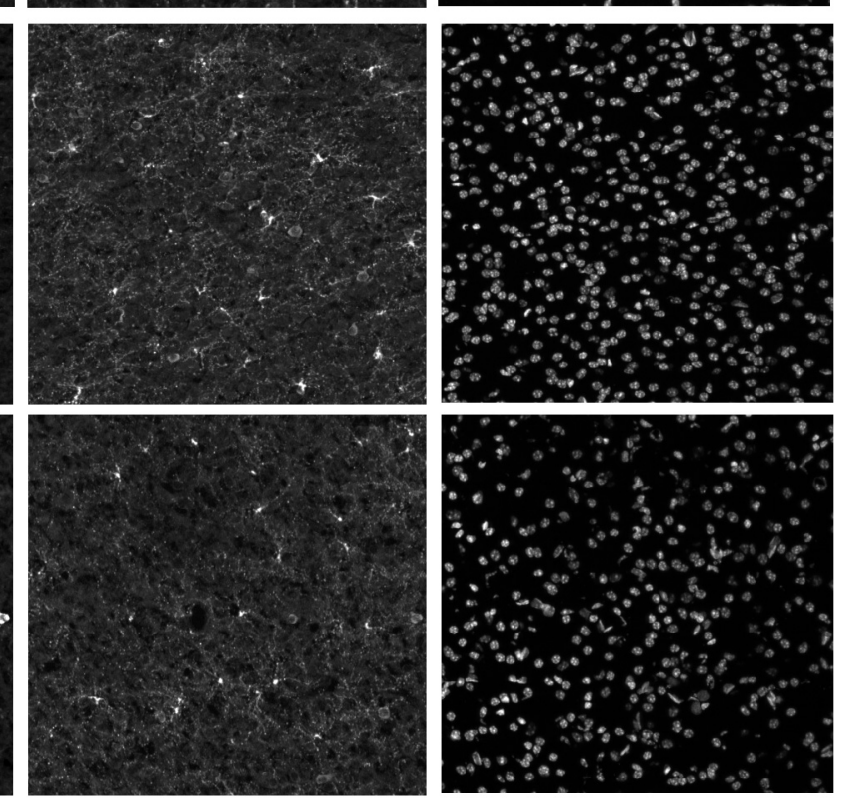


Figure S11: Efficient OPC-specific Tns3 deletion in the postnatal brain and reduced oligodendrocyte generation from OPCs. (A) Scheme of tamoxifen administration to Tns3-iKO and control $\left(\mathrm{Cre}^{+} ; \mathrm{Tns}^{+/+}\right)$mice, Cre-mediated genetic changes, and timing of experimental analysis. (B-B') Immunofluorescence for Tns3, GFP and Nkx2.2 in P21 mice sagittal brain sections at the level of the corpus callosum, illustrating the loss of Tns3 signal in Nkx2.2 $2^{\text {high }}$ iOL1s (arrows) but not in vessels (asterisk) of Tns3-iKO (B') compared to control (B). Note that Nkx2.2 $2^{\text {high }}$ iOL1s do not change in density in Tns3-iKO compared to control. (C) Histograms showing the Tns3+ cells density in the corpus callosum, fimbria, cortex, and striatum, in Tns3-

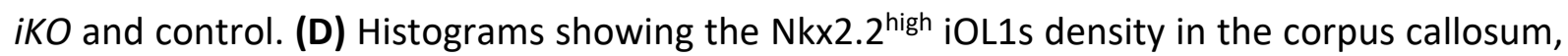
fimbria, cortex, and striatum, in Tns3-iKO and control. (E-F') Immunostaining corresponding to Fig.4B-C' panels showing individual channels for CC1, PDGFR $\alpha$, and DAPI in the fimbria (E,E') and cortex $\left(\mathbf{F}, \mathbf{F}^{\prime}\right)$ of control mice (E,F) and Tns3-iKO mice (E', $\left.\mathbf{F}^{\prime}\right)$. CC, corpus callosum. Scale bar, $20 \mu \mathrm{m}$. 
Figure S12
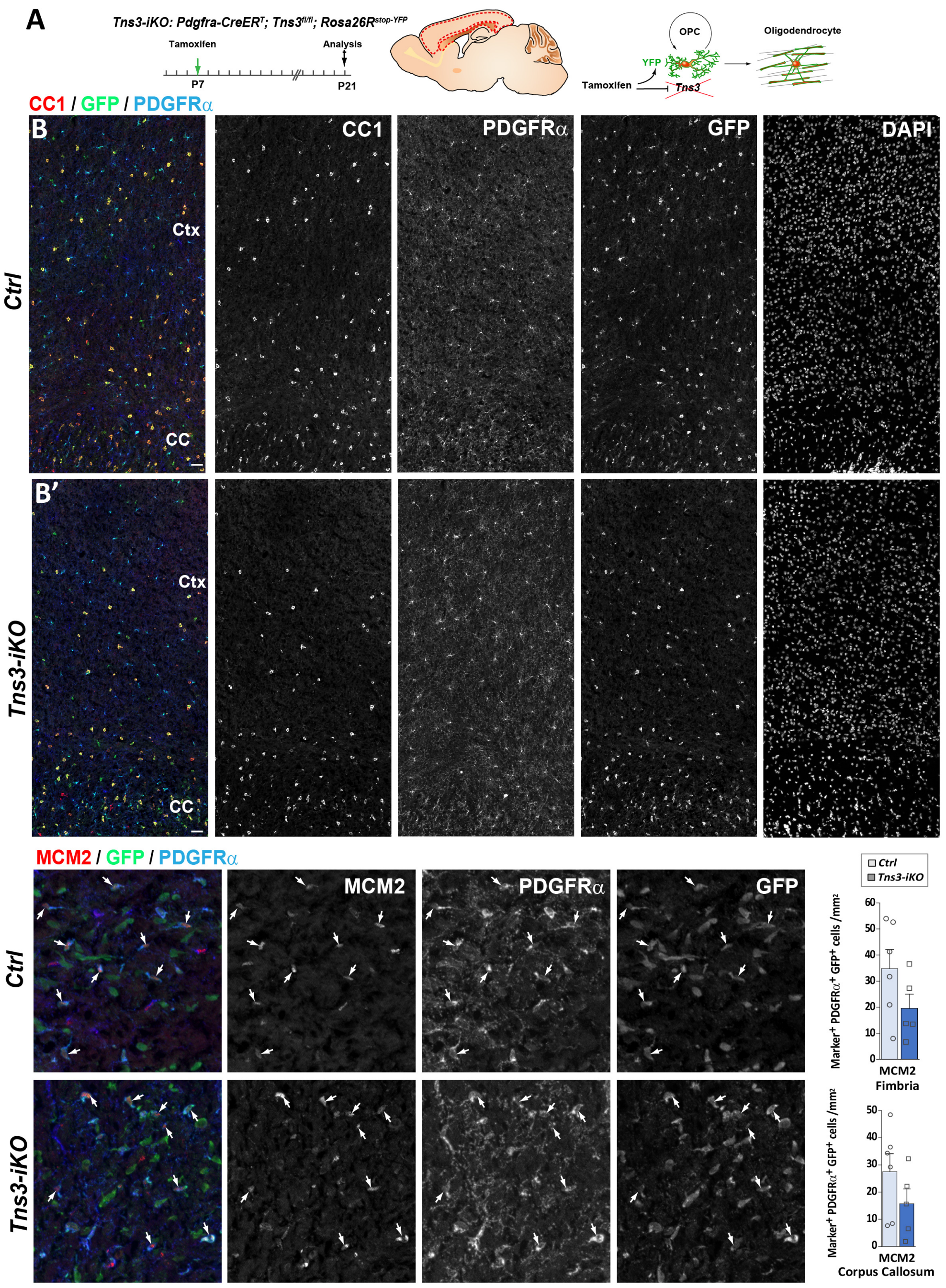


\section{Figure S12: OPC-specific Tns3 deletion reduces oligodendrocyte generation without} changing OPC proliferation in the postnatal brain. (A) Scheme of tamoxifen administration to Tns3-iKO and control $\left(\mathrm{Cre}^{+}\right.$; $\left.\mathrm{Tns}^{+/+}\right)$mice, Cre-mediated genetic changes, and timing of experimental analysis. (B-B') Immunostaining illustrating at low magnification the reduction in number of $\mathrm{CC}^{+}$OLs but not PDGFR $\alpha^{+}$OPCs in the cortex and corpus callosum of Tns3-iKO mice ( $\left.\mathbf{B}^{\prime}\right)$ compared to control mice (B), showing each individual channel and DAPI. (C-C'). Immunofluorescence for MCM2 proliferative marker, GFP, and PDGFR $\alpha$ illustrating similar proportion of proliferative OPCs in control (C) and Tns3-iKO mice (C'). (D) Histograms quantifying the proliferative fraction of GFP+ OPCs in the fimbria (Upper) and corpus callosum (Lower). Note the slight reduction (non-significant) in proliferation in Tns3-iKO OPCs compared to control OPCs. CC, corpus callosum; Ctx, cortex. Scale bar, $20 \mu \mathrm{m}$. 
Figure S13. Tns3 is co-expressed with integrin signaling genes in immature oligodendrocytes.

(A,B) Dotplots showing Tns3/TNS3 expression patter in mouse (A) and human (B) single cells at different stages of oligodendrogenesis, together with key markers of each cluster and genes involved in integrin signaling pathway. Note that high levels of Tns3/TNS3 in immature oligodendroctyes (iOL1 and $\mathrm{iOL2}$ ) together with known genes of integrin pathway previously implicated in oligodendrogenesis (Itgb1, Fyn, FAK/Bcar1, p130Cas/Ptk2) both in mouse and human cells. (C) Immunofluorescence in neurosphere-derived neural progenitor cultures generated from Tns3 ${ }^{\text {Tns3-v5 }}$ neonatal brain after 3 days of differentiation showing coexpression of Tns3-V5 (V5 antibody) and Integrin- $\beta 1$ in CNP ${ }^{+}$differentiating OLs (arrows). Scale bar, $20 \mu \mathrm{m}$. 